\title{
Numerical Design Optimisation for the Karoo Array Telescope
}

by

N.J.D. Joubert

Thesis presented in partial fulfilment of the requirements

for the degree of Master of Science in Mechanical

Engineering at Stellenbosch University

Department of Mechanical and Mechatronics Engineering,

University of Stellenbosch,

Private Bag X1, Matieland 7602, South Africa.

Supervisor: Prof. G. Venter

February 2009 


\section{Declaration}

I, the undersigned, hereby declare that the work contained in this thesis is my own original work and that I have not previously in its entirety or in part submitted it at any university for a degree.

Signature:

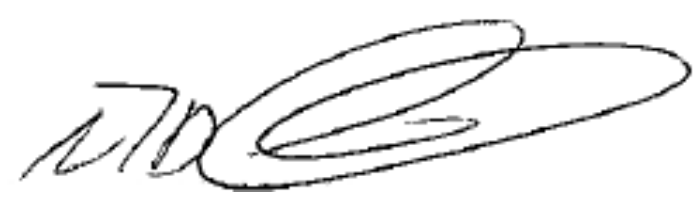

N.J.D. Joubert

Date: 9 February 2009

Copyright (C) 2009 Stellenbosch University

All rights reserved. 


\title{
Abstract
}

\section{Numerical Design Optimisation for the Karoo Array Telescope}

\author{
N.J.D. Joubert \\ Department of Mechanical and Mechatronics Engineering, \\ University of Stellenbosch, \\ Private Bag X1, Matieland 7602, South Africa. \\ Thesis: MScEng (Mech)
}

February 2009

Although mass minimisation is an important application within structural optimisation, other applications include: (1) concept generation, (2) concept evaluation, (3) design for structural feasibility and (4) data matching. These applications, except data matching, are discussed and illustrated on a prototype design of the Karoo Array Telescope (KAT) antenna. The KAT passed through the design process and a full scale prototype was built, but was found to be too expensive. A detailed finite element model of the finalised design was considered as a test bed for reducing costs.

Size-, shape- and topology optimisation are applied to three KAT components, while considering wind, temperature and gravity loads. Structural- and nonstructural constraints are introduced. Coupling of the structural optimisation code with an external analysis program to include non-structural responses and the parallelisation of the sensitivity calculations are presented.

It is shown that if a finite element model is available, it is generally possible to apply structural optimisation to improve an existing design. A reduction of $2673 \mathrm{~kg}$ steel was accomplished for the existing KAT components. The total cost saving for the project will be significant, when considering that a large amount of antennas will be manufactured. 


\title{
Uittreksel
}

\section{Numeriese Ontwerpsoptimering vir die "Karoo Array Telescope"}

\author{
N.J.D. Joubert \\ Departement Meganiese en Megatroniese Ingenieurswese, \\ Universiteit van Stellenbosch, \\ Privaatsak X1, Matieland 7602, Suid Afrika. \\ Tesis: MScIng (Meg)
}

Februarie 2009

Massa minimering is ' $\mathrm{n}$ belangrike toepassing van strukturele optimering. Daar bestaan egter ook ander toepassings, nl: (1) konsepgenerering, (2) konsepevaluasie, (3) ontwerp vir strukturele uitvoerbaarheid en (4) data passing. Hierdie toepassings, behalwe data passing, word bespreek en geïllustreer op 'n prototipe ontwerp van die "Karoo Array Telescope" (KAT) antenna. Die KAT is deur die hele ontwerpsproses en 'n volskaalse prototipe is gebou. Dit is egter as te duur bevind. 'n Gedetailleerde eindige element model van die gefinaliseerde ontwerp is gebruik as 'n maatstaf om die koste te verlaag.

Grootte-, vorm- en topologie optimering is aangewend op drie KAT komponente, met in agneming van wind-, temperatuur- en gravitasie belastings. Strukturele en nie-strukturele beperkings word toegepas. Die koppeling van 'n strukturele optimeringskode met 'n eksterne analiseprogram word aangetoon. Laasgenoemde word gebruik vir die berekening van nie-strukturele respons en die parallelisering van gradiënt berekeninge.

Daar word aangetoon dat dit oor die algemeen moontlik is om strukturele optimering toe te pas om 'n bestaande ontwerp te verbeter indien 'n eindige element model beskikbaar is. 'n Vermindering van $2673 \mathrm{~kg}$ staal is bereik vir die bestaaande KAT komponente. Die kostebesparing vir die hele projek sal noemenswaardig wees, aangesien 'n groot aantal antennas vervaardig moet word. 


\section{Contents}

Declaration $\quad$ i

Abstract

Uittreksel iii

Contents

List of Figures vii

List of Tables $\quad$ ix

Nomenclature $\quad$ xi

1 Introduction 1

2 Literature study $\quad 3$

2.1 Numerical design optimisation . . . . . . . . . . . . . . . . . 3

2.1.1 Advantages of numerical design optimisation . . . . . . . . 4

2.1.2 Limitations of numerical design optimisation . . . . . . . . 4

2.1.3 The general optimisation statement . . . . . . . . . 5

2.1.4 The iterative optimisation algorithm . . . . . . . . 5

2.2 Structural optimisation . . . . . . . . . . . . . . . . . . . . 6

2.2.1 Background . . . . . . . . . . . . . 6

2.2.2 Modern structural optimisation . . . . . . . . . . . 7

2.3 Structural optimisation techniques . . . . . . . . . . . . . 9

2.3.1 Size optimisation . . . . . . . . . . . . . . . . . . . 9 9

2.3.2 Shape optimisation . . . . . . . . . . . . . . . 11

2.3.3 Topology optimisation ............... . . 14

2.3.4 Topometry optimisation . . . . . . . . . . . 16

2.3.5 Topography optimisation . . . . . . . . . . . . . 17 
3 The Karoo Array Telescope 18

3.1 Background . . . . . . . . . . . . . . . . . . . . . 18

3.2 The structural design . . . . . . . . . . . . . . . . . . . . . . . . 19

3.3 Design specifications . . . . . . . . . . . . . . . . 20

4 The Finite element model 22

4.1 The ANSYS finite element model . . . . . . . . . . . . . . 22

4.2 Loadcases . . . . . . . . . . . . . . . . . . . . . . . . 23

4.3 Conversion of the finite element model . . . . . . . . . . . 25

4.3 .1 Load data . . . . . . . . . . . . . . . . . 26

4.3 .2 Incomplete mesh . . . . . . . . . . . . . . . . 28

4.3.3 Disconnected components . . . . . . . . . . . . . . . . . 29

4.3.4 Reduction in shell element order . . . . . . . . . . . . . 31

4.4 Finite element model comparison . . . . . . . . . . . . . . . 33

5 External program $\quad 39$

5.1 Overview of the external program . . . . . . . . . . . . 39

5.1 .1 The analysis section $\ldots \ldots \ldots \ldots$

5.1 .2 The gradient section . . . . . . . . . . . . . . 40

5.2 Least squares fit . . . . . . . . . . . . . . . . . . . . . . 42

5.3 Non-structural responses calculation . . . . . . . . . . . 45

5.4 Step-size calculation . . . . . . . . . . . . . . . . 48

5.5 Parallel processing . . . . . . . . . . . . . . . . . . . 49

6 KAT optimisation $\quad 55$

6.1 Optimisation problem definition . . . . . . . . . 55

6.1 .1 Objective function . . . . . . . . . . 55

6.1 .2 Constraints . . . . . . . . . . . . . 56

6.2 Mass minimisation . . . . . . . . . . . . . . . 58

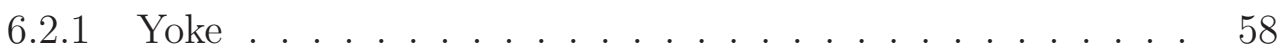

6.2 .2 Backing structure . . . . . . . . . . . . . . 60

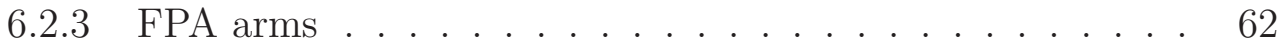

6.2.4 System level optimisation _. . . . . . . . . . . . . 64

6.3 Concept generation . . . . . . . . . . . . . . . . . 65

6.3.1 Topology optimisation . . . . . . . . . . . . 66

6.3.2 Backing structure - Some holes removed . . . . . . . . 68

6.3.3 Backing structure - All holes removed . . . . . . . . . . 70

6.3 .4 Yoke - Outer shell . . . . . . . . . . . . . . . . . . . 71

6.3.5 Yoke - Added stiffness _ . . . . . . . . . . . . . 72 
6.4 Concept evaluation . . . . . . . . . . . . . . . . . . 74

6.4.1 Backing structure . . . . . . . . . . . . . . . 74

6.4 .2 Yoke . . . . . . . . . . . . . . . . 75

6.5 Design for structural feasibility . . . . . . . . . . . . 76

7 Conclusion $\quad \mathbf{8 0}$

7.1 Future work . . . . . . . . . . . . . . . . . 80

7.2 Conclusion . . . . . . . . . . . . . . . . . . . . . 81

$\begin{array}{lr}\text { Bibliography } & 84\end{array}$ 


\section{List of Figures}

2.1 Modern structural optimisation programs . . . . . . . . . . 8

2.2 Three rod truss . . . . . . . . . . . . . . . . . . . . . . 10

2.3 The change in rod areas for the three rod truss . . . . . . . . . . 11

2.4 Initial shape and basis vector . . . . . . . . . . . . . . . . . 12

2.5 Quad4 domain element . . . . . . . . . . . . . . 13

$2.6 \quad 18$-rod truss . . . . . . . . . . . . . . . . . . . 13

2.7 Initial- and optimised shape for the 18-rod truss . . . . . . . . . . . 14

2.8 The change in rod areas for the 18-rod truss . . . . . . . . . . . . . 14

2.9 Rectangular block of material . . . . . . . . . . . . . . . . 16

2.10 Results of the topology optimisation . . . . . . . . . . . 16

3.1 The prototype KAT antenna . . . . . . . . . . . . . . . 19

3.2 Components of the KAT . . . . . . . . . . . . . . . . 20

4.1 The ANSYS finite element model of the KAT . . . . . . . . . . . 23

4.2 A comparison of the temperature loads between the GENESIS- and ANSYS models . . . . . . . . . . . . . . . . . 27

4.3 Incomplete mesh of the azimuth ring gear . . . . . . . . . . . 28

4.4 Displacements on the pedestal due to a $60{ }^{\circ} \mathrm{C}$ temperature load . . 29

4.5 Rigid elements inserted into the finite element model . . . . . . . . 30

4.6 Shell element Von Mises stress for the GENESIS model with the original- and refined mesh under gravity loading . . . . . . . . . . . 32

4.7 KAT finite element model . . . . . . . . . . . . . . . . . . . . . 33

4.8 A comparison of displacements from temperature loads between the GENESIS- and ANSYS models . . . . . . . . . . . . . . . . . 35

4.9 A comparison of the first five natural modes between the GENESISand ANSYS models . . . . . . . . . . . . . . . . 36

4.10 A comparison between the natural frequencies of the ANSYS- and GENESIS models and two cases of rigid element removal . . . . . . 37 
5.1 Coupling of GENESIS with the external program . . . . . . . 40

5.2 The analysis section of the external program . . . . . . . . . 40

5.3 The gradient section of the external program . . . . . . . . . 43

5.4 Three least squares fits with different levels of compensation . . . . 44

5.5 An Illustration of the dish surface error calculation . . . . . . . 46

5.6 An Illustration of the relative FPA displacement calculation . . . 47

5.7 Yoke optimisation with a different number of processors . . . . . . 51

5.8 Yoke optimisation with different levels of parallelisation . . . . . . 52

5.9 Plots of the objective function and maximum constraint violation versus design cycles for the serial- and parallel processing . . . . . 53

5.10 A comparison between serial- and parallel processing for various cases of optimisation . . . . . . . . . . . . . . . 54

6.1 Original yoke with the colours indicating different properties .... 59

6.2 Design variables for size optimisation of the original yoke . . . . . 60

6.3 The backing structure . . . . . . . . . . . . . 60

6.4 Design variables for size optimisation of the original backing structure 61

6.5 The shape changes made to the backing structure . . . . . . . . 62

6.6 Original FPA arms and dish with the colours indicating different properties .......................... 63

6.7 FPA arm's shape before- and after shape optimisation . . . . . . . . 64

6.8 Design variables for size optimisation of the original FPA arms . . . 64

6.9 The backing structure without holes . . . . . . . . . . . . 67

6.10 Retained material and removed material for the four cases of topology optimisation . . . . . . . . . . . . . . . 6 67

6.11 Backing structure with some of the holes removed (Concept B2) . . 68

6.12 Design variables for size optimisation of the backing structure (Con-

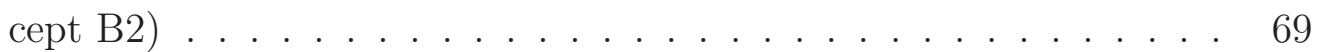

6.13 Proposed improvement on Concept B2 . . . . . . . . . . . 70

6.14 Design variables for size optimisation of the backing structure (Con-

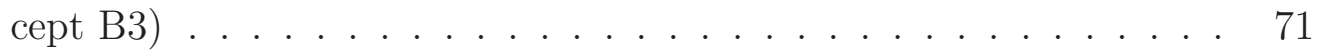

6.15 Two new concepts for the yoke . . . . . . . . . . . 73

6.16 Design variables for size optimisation of the yoke (Concept Y3) . . . 73

6.17 Visualisation of minimising the maximum constraint violation with

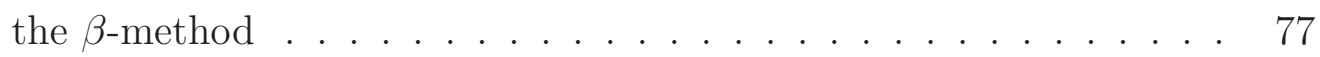




\section{List of Tables}

2.1 Mass results for the three $\operatorname{rod}$ truss $\ldots \ldots \ldots \ldots$

2.2 Mass results for the 18 -rod truss . . . . . . . . . . . . . . 14

2.3 Strain energy results for the topology optimisation . . . . . . . . . 16

3.1 Normal and survival environments . . . . . . . . . . . . . . . . . 21

4.1 Loadcases in the finite element model . . . . . . . . . . . . . . 24

4.2 The temperature values of the temperature loads depicted in Figure 4.227

4.3 Displacements of the pedestal for various loads . . . . . . . . . . 28

4.4 The magnitude displacement and natural frequency variances between the GENESIS finite element model with the original- and refined mesh . . . . . . . . . . . . . . . . . . . . . 32

4.5 Displacement ranges for the GENESIS and ANSYS models . . . . . 34

4.6 Corrected displacement ranges for the GENESIS and ANSYS models 35

4.7 Natural frequencies for the GENESIS and ANSYS models . . . . . 37

5.1 A comparison of the results from the least squares fits . . . . . . . 44

5.2 A comparison of the relative FPA displacements and rotations . . . 48

6.1 Bounds on the stresses in each component . . . . . . . . 57

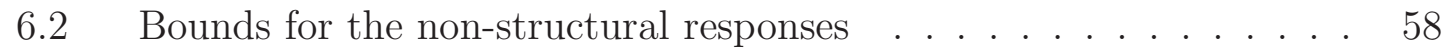

6.3 Mass results for the original yoke . . . . . . . . . . . . . 59

6.4 Mass results for the original backing structure . . . . . . . . . . 62

6.5 Mass results for the original FPA arms . . . . . . . . . . . 63

6.6 Comparison between component and system level optimisation . . . 65

6.7 Mass results for the backing structure (Concept B2) . . . . . . . 69

6.8 Mass results for the backing structure (Concept B3) . . . . . . . 71

6.9 A comparison of the initial- and least infeasible design point for the

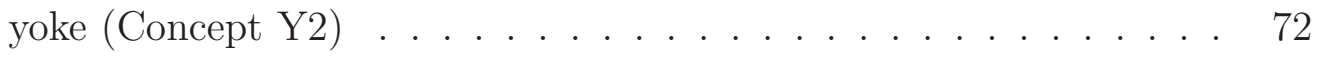

6.10 Mass results for the yoke (Concept Y3) . . . . . . . . . . 73

6.11 Comparison between the different concepts for the backing structure 74 
6.12 Comparison between the different concepts for the yoke . . . . . . 75

6.13 A comparison of the initial- and least infeasible design points for the KAT . . . . . . . . . . . . . . . . . . . . . 78

6.14 A comparison of the initial- and least infeasible non-structural responses . . . . . . . . . . . . . . . . . . 


\title{
Nomenclature
}

\author{
Optimisation \\ F $\quad$ Function \\ $g \quad$ Inequality constraint \\ $h \quad$ Equality constraint \\ S Search direction \\ $X \quad$ Design variable \\ $\beta \quad$ Artificial design variable
}

\section{Variables}

A Area

$b \quad$ Tilt around the $y$-axis

C Zernike polynomial coefficient

$c \quad$ Tilt around the $z$-axis

$C_{d} \quad$ Drag coefficient

$D S E \quad$ Dish surface error

$d \quad$ Bias along the $x$-axis

$f_{d} \quad$ Focal distance

$f f_{d} \quad$ Focal distance of the fitted paraboloid

$\triangle f_{d} \quad$ Difference in focal distances

$H \quad$ Height

I Moment of inertia

$P \quad$ Pressure

$q \quad$ Dynamic pressure

$R X \quad$ Rotation around the $x$-axis

$R Y \quad$ Rotation around the $y$-axis

$R Z \quad$ Rotation around the $z$-axis 
$T X \quad$ Translation along the $x$-axis

TY Translation along the $y$-axis

$T Z \quad$ Translation along the $z$-axis

$u f_{d} \quad$ Undeformed focal distance

$v \quad$ Velocity

W Width

$x \quad$ Coordinate along the $x$-axis

$Y Z \quad$ Displacement in the $Y Z$-plane

$y \quad$ Coordinate along the $y$-axis

Z Zernike polynomial

$z \quad$ Coordinate along the $z$-axis

$\rho \quad$ Density

$\phi \quad$ Zernike polynomial orientation

\section{Superscripts}

$i \quad$ Grid point number

$q \quad$ Iteration number

\section{Subscripts}

$\begin{array}{ll}F P A & \text { Focal Plane Array } \\ f p & \text { Focus point } \\ \max & \text { Maximum } \\ r e l & \text { Relative } \\ r m s & \text { Root mean square } \\ x & \text { Around the } x \text {-axis } \\ 0 & \text { Initial design point }\end{array}$




\section{Chapter 1}

\section{Introduction}

This thesis provides an overview of the application of structural optimisation to a real-life engineering design problem. The problem considered is the design of a radio telescope, called the Karoo Array Telescope (KAT), using a detailed finite element analysis. Different aspects of structural optimisation are introduced, discussed and illustrated, using the design problem as a test bed.

At this stage a prototype of the KAT has been designed and built at the Hartebeesthoek Radio Astronomy Observatory near Johannesburg, South Africa. The construction cost of the antenna was too high and a means of reducing the cost became necessary. Structural optimisation will be used as a design tool to modify the KAT prototype design, with the goal of reducing cost.

Within structural optimisation, the optimisation problem is most often formulated to minimise the mass of a structure. Although mass minimisation is an important application, other applications include: (1) concept generation, (2) concept evaluation, (3) design for structural feasibility and (4) data matching.

Mass minimisation, concept generation and concept evaluation are closely related. One has to consider the end goal of the designer to distinguish between these three structural optimisation applications. For mass minimisation the objective is to obtain a structure that is as light as possible, while satisfying all design constraints. Typically shape and/or size optimisation is used. Concept generation is used to create new concepts and is typically used early on in the design process. Normally, a solid block of material is used as a starting point, forces and boundary conditions are applied and topology optimisation is used to generate the new concept. Concept evaluation is an important use of optimisation that is frequently overlooked. The goal is to compare a set of optimal designed concepts. It is often meaningless to compare non-optimised concepts. 
The design for structural feasibility is the use of optimisation to find a design that satisfy all the design requirements or constraints. The focus is to obtain a feasible design of a structure that is initially infeasible. Often, mass minimisation and structural feasibility design are considered simultaneously.

Data matching is used when a correlation between a numerical model (e.g. finite element model) and experimental data is required. This is traditionally a manual process which is a tedious and time consuming task. Instead, optimisation can be used to automatically change the numerical model to best fit the experimental data. A good application can be found in [1] where structural optimisation is used to correlate experimental test data with a finite element model for a Cessna airplane wing.

The primary goal of this thesis is to demonstrate mass minimisation, concept generation, concept evaluation and design for structural feasibility by implementing the structural optimisation software, GENESIS [2]. Note that much of the functionality provided by GENESIS is also available in other commercial software codes. The present work will focus on the application and use of structural optimisation in general, rather than on any specific software.

The secondary objective will be to reduce the cost of the KAT, which went through the traditional design process. The focus will be to produce a lighter structure consisting of less material, which will result in decreased material costs.

A shortcoming of current structural optimisation software is that the manufacturing cost can not be inserted as a constraint. Ultimately, the manufacturing cost will not directly be taken into consideration during the optimisation process. During concept generation and concept evaluation an attempt will be made to develop designs that are cheaper to manufacture than the prototype.

In this thesis, a literature study on numerical design optimisation and structural optimisation are provided. Thereafter, the background and design specifications for the KAT are presented. The conversion and modifications of the finite element model (due to information loss during the conversion) will be discussed. A comparison between the output from the ANSYS- and GENESIS finite elements models are presented. The coupling of the structural optimisation code with an external analysis program, to include non-structural responses, will also be illustrated. A two level parallelisation is demonstrated by making use of shared- and dynamic memory parallel processing. The optimisation problem definition for the KAT will be presented. Thereafter, the structural optimisation applications will be illustrated on the KAT antenna. 


\section{Chapter 2}

\section{Literature study}

Engineering design is quantifiable, which enable the use of numerical design optimisation and computers to quickly search through a great many alternative designs. Numerical design optimisation provides a powerful tool that aids the designer in the design process, by having the computer do the repetitive tasks. This chapter provides an overview of numerical design optimisation and structural optimisation in particular, focusing specifically on the different structural optimisation techniques.

\subsection{Numerical design optimisation}

The traditional approach to design [3] was to use graphs and charts, that were developed over many years of experience in order to increase the knowledge about the problem. These methods were mostly efficient to give a feasible solution.

As the design problem increases in complexity, designers make use of computers for detailed numerical analysis [3]. The computer analysis code can give a measure of the feasibility and optimality of the proposed design, much faster than hand calculations or experiments. The designer can then easily change the design variables (the parameters that are allowed to change during the design) and rerun the analysis to determine the influence on the design. Analyses are executed for a wide range of the design variables and the results are plotted to obtain a relationship between the design parameters and the objective function and constraint values. The designer can then use these graphs to find an optimum design by interpolating or extrapolating. This is an approach that is effective only if the number of design parameters is small. If there is more than three variables in the design it quickly becomes very difficult to work with graphs. An alternative is to automate the whole design process. 
Numerical design optimisation provides a logical structure for such an automated design process. The designer only have to concentrate on providing the correct input and evaluating and interpreting the output, while the repetitive tasks are allocated to the computer. The use of numerical design optimisation typically helps the designer to gain more insight into the problem, reduces the design time and results in an improved design. The advantages and limitations of numerical design optimisation will be addressed in the following paragraphs.

\subsubsection{Advantages of numerical design optimisation}

The advantages of numerical design optimisation can be summarised as [3]:

- A reduction in the design time (especially if one compares it to the traditional methods discussed in the previous paragraphs).

- A systematic and logical design procedure.

- A large number of design variables and constraints can be considered.

- An improvement in the design quality.

- The possibility that it can provide improved, non-traditional results compared to those anticipated by applying engineering intuition or experience.

- The interaction between the designer and the computer are limited to the minimum.

\subsubsection{Limitations of numerical design optimisation}

The limitations of numerical design optimisation also have to be considered [3]:

- The increase in computational time with an increase in the number of design variables. Typically, the computational time is an order of magnitude larger than that of a single analysis. It may be impossible to account for all possible design parameters if the cost of automated design becomes too expensive.

- Only a model of the real design is analysed and the optimisation results should always be checked. Optimisation can easily take advantage of analysis errors if the analysis is not accurate. This could lead to a meaningless or even a dangerous design. 
- There is no assurance that the optimisation algorithm will obtain a global optimum. Many times optimisation has to be restarted from various design points to ensure a global optimum.

\subsubsection{The general optimisation statement}

Many forms exists for stating the optimisation problem. One of the most commonly used forms for the non-linear constrained optimisation problem [3] is as follow:

Minimise:

$$
F(\mathbf{X})
$$

Subject to:

$$
\begin{array}{cc}
g_{i}(\mathbf{X}) \leq 0 & i=1,2, \ldots, p \\
h_{j}(\mathbf{X})=0 & j=1,2, \ldots, m \\
X_{k}^{l} \leq X_{k} \leq X_{k}^{u} & k=1,2, \ldots, l
\end{array}
$$

where

$$
\mathbf{X}=X_{1}, X_{2}, \ldots, X_{l}
$$

The goal is to minimise the objective function (2.1.1) by determining the best combination of design variables (2.1.5). Certain requirements (constraints) must be met for the design to be acceptable (2.1.2) and (2.1.3). The upper and lower bounds of the design variables (2.1.4) are referred to as side constraints and defines the searchable design space.

\subsubsection{The iterative optimisation algorithm}

Many gradient-based optimisation algorithms make use of an iterative procedure [3] to determine the optimum of a function. An initial set of design variables is required as a starting point. The design variables are then updated, iteratively until the optimum is reached. This iterative procedure [3] can be summarised as:

$$
\mathbf{X}^{q+1}=\mathbf{X}^{q}+\alpha \mathbf{S}^{q}
$$

In (2.1.6), $q$ is the iteration number, $\mathbf{X}^{q}$ is the current design point and $\mathbf{X}^{q+1}$ is the updated design point after one iteration. $\mathbf{S}^{q}$ is the direction in which the optimiser will search and $\alpha$ is a scalar parameter indicating how far the optimiser should move in the search direction. For the first iteration in (2.1.6), the starting point $\left(\mathbf{X}^{\mathbf{0}}\right)$ is provided by the designer. This design point does not have to be 
feasible. The only unknowns in (2.1.6), are the search direction and the scalar parameter, $\alpha$, which have to be determined to calculate the next design point.

The search direction $\left(\mathbf{S}^{q}\right)$ is calculated first, which will result in two situations depending on the feasibility of the design point. If the current design is feasible, then the optimiser will chose a search direction that will decrease the objective function without violating any constraints. On the other hand, if the current design is infeasible, then the optimiser will choose a search direction that will point towards a feasible design without regards to the objective function. Generally the primary focus of an optimiser is firstly to find a feasible design. Only after finding a feasible design will the optimiser try to decrease the objective function.

Next, the optimiser has to determine how far to search in the search direction. This step is known as the "one-dimensional search". During the one-dimensional search, the optimiser has to find the optimum value for $\alpha$ to improve the design as much as possible. Again there are two cases that the optimiser has to look at depending on the feasibility of the design. If the current design is feasible, the value of $\alpha$ has to be such that the objective function will be minimised without violating any constraints. If the current design is infeasible, then $\alpha$ has to be chosen such that the design will be feasible or as near the feasible region as possible.

After the search direction and the scalar parameter $\alpha$ has been calculated, it is possible to determine the next design point using (2.1.6). This two step process is followed until convergence. For further information regarding the optimisation process, see [3].

\section{$2.2 \quad$ Structural optimisation}

The technology associated with structural optimisation developed considerably during the past few decades. First, a short history of structural optimisation will be provided, followed by a discussion of the modern technology used for structural optimisation.

\subsubsection{Background}

Structural optimisation originated from the early work of Maxwell [4] in 1869 and Michell [5] in 1904. Substantial analytical work were done on component optimisation in the 1940's and 1950's. This included the weight strength analysis

of aircraft structures by Shanley [6]. Thereafter the combination of the development of linear programming techniques and the coming of the digital computer 
lead to the application of mathematical programming to the design of beam and frame structures, e.g. Heymann [7].

In 1960, Schmit showed that structural analysis can be integrated with optimisation [8]. He solved the structural design problem by combining numerical optimisation with finite element analysis. During the sixties and early seventies optimisation was seen as the coupling of finite element analysis, sensitivity analysis and optimisation [3]. A finite element analysis was done whenever the optimiser needed to evaluate the objective function and constraints. The problem with this technique is that the computational cost increase as more finite element analyses are performed. Therefore the number of finite element analyses had to be limited to reduce the computational cost.

Schmidt and his co-workers [9] introduced approximation techniques for structural optimisation to reduce the number of finite element analyses and thus the computational cost. Schmidt and Miura [10] refined these concepts. They could create high quality approximations for the initial design problem that could be used during the optimisation process. This approach lead to the possibility of an optimum design at much lower computational cost.

In the eighties Vanderplaats and Salajegheh [11], and Canfield [12] refined the concepts even more, resulting in more efficient approximations. These approximations are referred to as "second generation approximations" and are presently still used.

\subsubsection{Modern structural optimisation}

Modern structural optimisation programs reduce the number of finite element analyses, which result in lower computational cost. These programs do not provide a simple coupling of a finite element analysis with sensitivity analysis and optimisation, but it introduces the concept of approximations [3]. An example of a modern structural optimisation program is illustrated in Figure 2.1.

The overall process [3] consists of an outer loop and an inner loop as shown in Figure 2.1. The outer loop consists of the finite element analysis, constraint screening, sensitivity analysis and the approximate problem generator. The inner loop consists of the approximate analysis and an optimiser which solves the approximate problem. A cycle through the inner loop is typically referred to as an optimisation iteration, while a cycle through the outer loop as a design cycle. A finite element analysis and gradient calculations for the retained responses are necessary during each design cycle. It is critical that the approximations are of high quality and are quickly evaluated to reduce the computational cost. The 


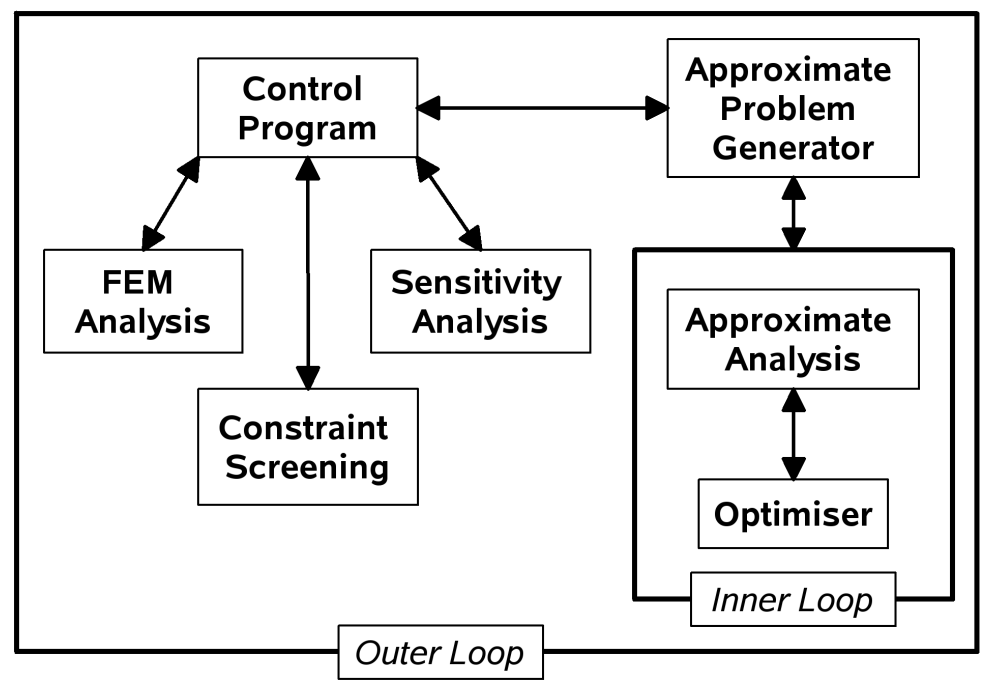

Figure 2.1: Modern structural optimisation programs [3]

methods implemented in modern structural optimisation programs [10] illustrated in Figure 2.1, will be described in the subsequent paragraphs.

Firstly, a full finite element analysis of the initial design is performed. All constraints are evaluated and constraint screening [3] is performed where only the critical or near critical constraints are kept. During constraint screening all constraints that are far from their respective bounds are ignored. For example, a constraint is discarded when it is more than $10 \%$ from its bound. This technique improves the efficiency and lower the computational cost of the optimisation.

Next, a sensitivity analysis [3] is performed where the gradients of the objective function and the constraints that were retained after the constraint screening, are calculated. If the finite element mesh are very fine, then the elements in small regions of the structure will have nearly the same stresses and displacements. Only the most critical of these stresses or displacements in a specific region are retained in the approximation. It is unnecessary to retain all the stresses or displacements as the most critical response will represent the responses in that region. Although the structure may consist of a large number of constraints, only a small fraction are retained for sensitivity and approximate optimisation. The constraint reduction does not influence the final optimum point and is only implemented to lower the computational effort.

With the function value and gradient information available, the initial optimisation problem is approximated, by using the gradients of the retained constraints, and is solved by a general optimisation code [3]. The solution of the approximate optimisation is used to update the full model. By implementing the approximate 
approach to evaluate the objective function or constraints, instead of performing a full finite element analysis, one finds that the number of analyses are reduced by an order of a magnitude [13].

By using approximation techniques, one has to limit the amount by which the design variables can change during a design cycle. This technique is termed move limits [3] and normally restricts the design variables to change by no more than $50 \%$ during a design cycle. Move limits guard against unreasonably large perturbations in the design variables and ensure a valid approximation. If the design variables exceed their move limits, it is necessary to perform a finite element analysis for the new design. The move limits are not applicable when the design converges, but it is important during the beginning of the design to direct the design process.

The last step is to update the analysis data with the results from the approximate optimisation. Thereafter the analysis program are called to perform a finite element analysis. These results are compared with the approximated design. The program will terminate if either of three convergence criteria are met [2]. First, if the user defined number of design cycles have been reached. Secondly, if no progress was made in the approximate optimisation i.e. the design variables did not change. Lastly, if the objective function has not been improved considerably after two consecutive design cycles.

\subsection{Structural optimisation techniques}

For this project, GENESIS will be used as the optimiser and Design Studio for Genesis (DSG) as the pre- and post processor. GENESIS can implement five structural optimisation techniques used for structural design namely: size-, shape-, topology-, topometry- and topography optimisation. Each of these structural optimisation techniques will be discussed in the following paragraphs. Only size-, shape- and topology optimisation will be implemented in this study. An example problem will also be presented from literature for size-, shape- and topology optimisation. Note that these examples may not have a good starting point from an engineering point of view and are used for illustration purposes only.

\subsubsection{Size optimisation}

During size optimisation [14], the cross sectional dimensions (thickness of a shell element or the cross section of a bar element) are used as design variables. In finite element models, data is only provided for properties (area, moment of inertia, etc. 
for a finite element). A relationship between the cross sectional dimensions and the properties of a finite element is created to link the design variables with the finite element properties. This can be illustrated by choosing the height and the width of a bar element with rectangular cross section as the design variables. The area and second moment of inertia are related to the width and height by the following equations:

$$
\begin{gathered}
A=A(W, H)=W H \\
I_{x}=I_{x}(W, H)=\frac{W H^{3}}{12}
\end{gathered}
$$

With these equations, the optimiser has the freedom to change the width and the height of a finite element which will result in the change of the element properties. These relationships are used to optimise the objective function.

To show a simple example of size optimisation, consider the classical three rod truss shown in Figure 2.2. This example was acquired from the GENESIS User Manual [15] and all values had been changed to SI-units. The objective function is to minimise the mass, subject to stress constraints for each rod and displacement constraints at grid 4. Two loadcases are applied to the structure. The design variables are the cross section of each rod.

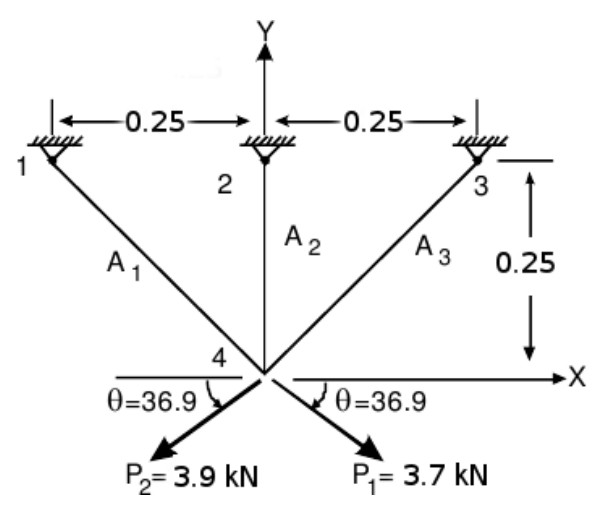

Figure 2.2: Three rod truss [15]

The three rod truss was optimised and the results are shown in Table 2.1 and Figure 2.3. After 17 design cycles the mass decreased by $39 \%$ or $96 \mathrm{~kg}$ with all constraints being met. The area of the middle rod decreased the most as displayed in Figure 2.3. This indicates that it contributes the least to the structure's stiffness compared to the other design variables. However, the middle rod can not be omitted from the structure as it is not at the lower bound, indicating that 
the mass will not be further reduced by removing it from the structure. The final structure is not symmetric because of the non-symmetric loading.

Table 2.1: Mass results for the three rod truss

\begin{tabular}{lc}
\hline Parameter & Value \\
\hline \hline Number of design cycles & 17 \\
Original mass (kg) & 244 \\
Optimised mass (kg) & 148 \\
\hline
\end{tabular}

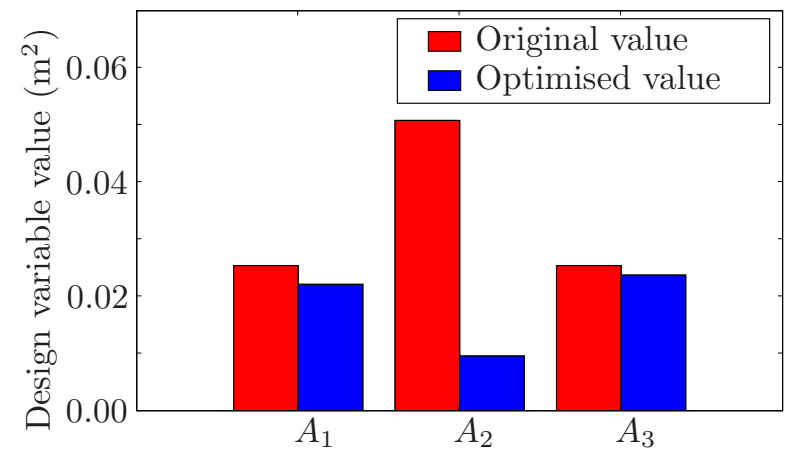

Figure 2.3: The change in rod areas for the three rod truss

\subsubsection{Shape optimisation}

Shape optimisation refer to a change in grid locations of a finite element model. Alternative shapes of the structure are required and can be obtained from a set of displacement vectors, called basis vectors. The goal of the optimiser is to find a linear combination of the basis vectors that represents the optimal design.

Figure 2.4 illustrates the concept of a basis vector by showing the difference between the initial shape and the basis vector of a rectangular structure. The value for the design variable can be one of the following cases [16]:

- It can be zero, which implies that the structure's length will remain unchanged.

- It can have a value of between zero and one. An interpolation between the original node locations and the basis vector will give the new shape of the structure. 


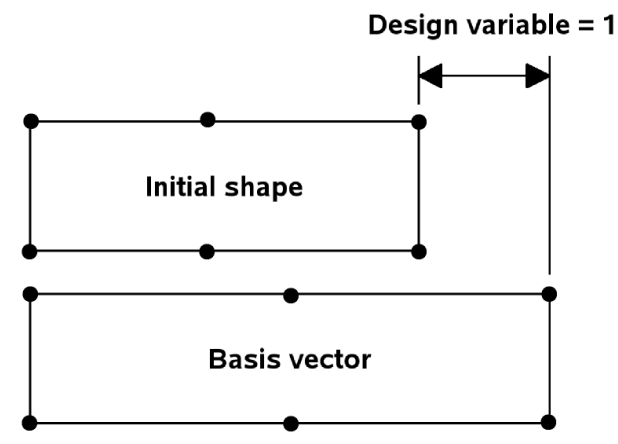

Figure 2.4: Initial shape and basis vector [16]

- It can have a value that is smaller than zero or greater than one. For this case the new shape is an extrapolation of the basis vector.

There are several ways to generate basis vectors including the grid basis vector method [17], the natural basis vector method [18] and the domain method [19]. The domain method was used to implement shape optimisation and will be discussed in more detail.

Before discussing the domain method, one should first look at the definition of a perturbation vector. In mathematical terms, a perturbation vector is the vectorial difference between the original grid locations and a basis vector [19]. In other words, the perturbation vector points to where the grids that is associated to it, would move if the corresponding design variable is one and all the other design variables are zero.

During the domain method [16], geometric regions (referred to as domain elements) are created which define the node interpolation functions that the optimiser is allowed to change. A domain element is basically a large finite element with many internal grids. A perturbation vector is applied to corner and/or midside nodes of the domain which constructs a basis vector. These perturbations are used with the domain element's shape functions to generate the perturbations of the internal nodes. Therefore a domain element act as a "rubber band" that can contract or stretch the borders of a structure. In Figure 2.5(a) a QUAD4 domain is shown with corner nodes 1, 4, 13 and 16. A perturbation is applied at node 4 and results in Figure 2.5(b). Here it can be seen that the interior nodes of the element are changed in proportion to the shape functions of the domain element.

Consider the 18-rod truss as shown in Figure 2.6, to illustrate shape optimisation. This example was acquired from the GENESIS User Manual [15] and all values had been changed to SI-units. In this example, size optimisation is also 


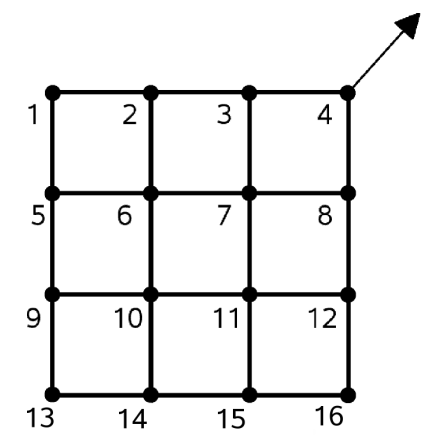

(a) Input data

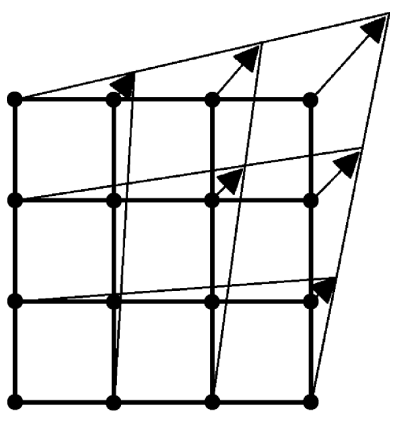

(b) Perturbation automatically generated

Figure 2.5: Quad4 domain element [19]

added to illustrate that size- and shape optimisation can be executed at the same time. The objective function is to minimise the mass, subject to stress- and Euler buckling constraints for each member. The structure is constrained at grids 10 and 11 and five point loads of $89 \mathrm{kN}$ each, are applied on grids $(1,2,4,6,8)$. Four size design variables were defined namely the areas of the top- $(1,4,8,12$, 16), the bottom- $(2,6,10,14,18)$, the vertical- $(3,7,11,15)$ and the diagonal $(5,9,13,17)$ members. Eight shape design variables were defined which control the $X$ and $Z$ movements of the bottom grids $(3,5,7,9)$.

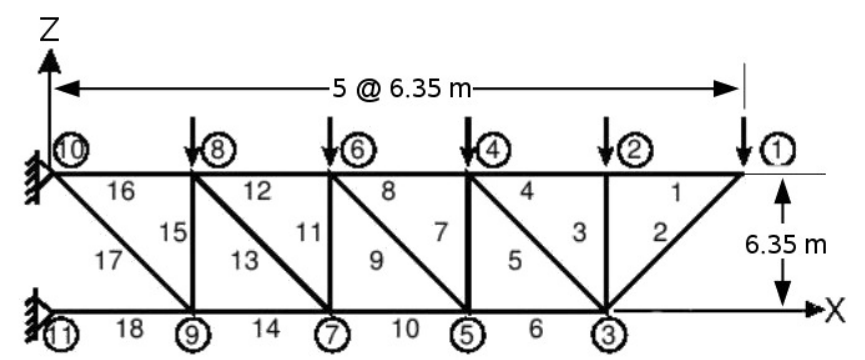

Figure 2.6: 18-rod truss [15]

Size- and shape optimisation were applied to the structure and the results are shown in Table 2.2 and Figures 2.7 and 2.8. After 5 design cycles the mass increased by $3 \%$ or $852 \mathrm{~kg}$ with all constraints being met. The reason for the increase in mass is that the initial structure had a constraint violation of $409 \%$ (the initial structure was infeasible). This shows one application of structural optimisation as it can re-design an infeasible design into a feasible design. In Figure 2.7, the initial shape (blue) versus the optimised shape (red) can be seen. Figure 2.8 shows the change in rod areas. $A_{\text {diagonal }}$ has reached its lower bound $(25.4 \mathrm{~mm})$ which brings to question if it should be omitted from the structure. The lower 
bound was decreased (to $1 \mathrm{~mm}$ ) to determine if the structure's mass will be further reduced by removing the rods corresponding to $A_{\text {diagonal }}$. Re-optimisation resulted in the design variable $(3.7 \mathrm{~mm})$ being larger than the decreased lower bound, indicating that it can not be omitted from the structure.

Table 2.2: Mass results for the 18-rod truss

\begin{tabular}{lc}
\hline Parameter & Value \\
\hline \hline Number of design cycles & 5 \\
Original mass (kg) & 32581 \\
Optimised mass $(\mathrm{kg})$ & 33433 \\
\hline
\end{tabular}

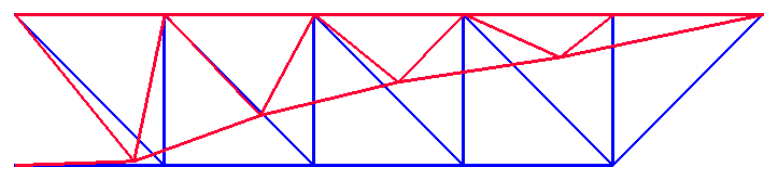

Figure 2.7: Initial- (blue) and optimised (red) shape for the 18-rod truss

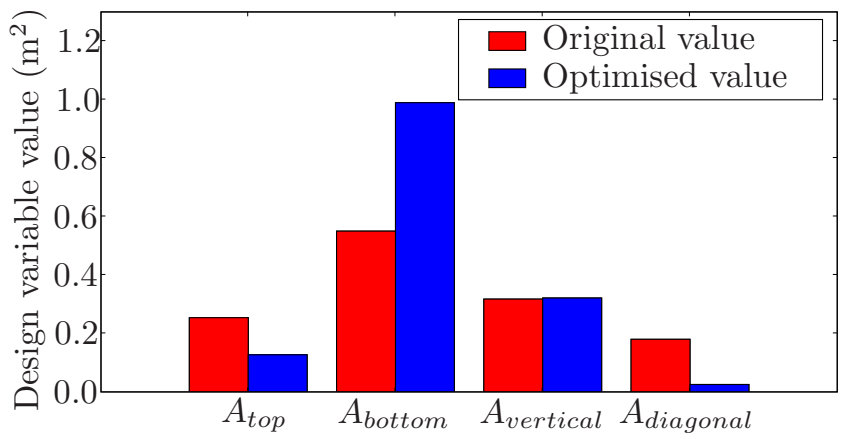

Figure 2.8: The change in rod areas for the 18-rod truss

\subsubsection{Topology optimisation}

Topology optimisation can generate completely new and innovative designs [20]. It is typically used to find a preliminary or conceptual design whereas shape- and size optimisation create detailed or final designs [21].

Topology optimisation [22], identifies regions of a structure that contribute least to the stiffness or the natural frequency, based on the objective function chosen by the user. Typically a solid block of material with a large number of 
finite elements is considered initially. Certain responses can be constrained including displacement, mass fraction, natural frequency etc., depending on the goal of the optimisation study. One of the most common constraints is to constrain the fraction of the initial mass that should be retained in the final design. This mass fraction constraint causes the optimiser to remove material which contributes the least to the structure's stiffness or natural frequency. The optimiser will ensure that the structure is as stiff as possible while keeping only the specified fraction of the original mass.

Topology optimisation can not be implemented simultaneously with sizeand/or shape optimisation. After topology optimisation has been performed, the designer must modify the finite element model, by interpreting and applying the results from the topology optimisation. Thereafter size- and shape optimisation are implemented to obtain a final design.

Topology optimisation can be divided into two general approaches [20]. The first approach attempts to find the microstructure parameters (e.g. size and orientation of holes) of each element in the designable region [23; 24]. For the second approach an optimal material distribution is found, by heuristically designing each finite element's material properties (e.g. Young's modulus, density) $[25 ; 26 ; 27]$. The second approach is used by GENESIS and it will be discussed in more detail.

During topology optimisation, GENESIS creates design variables that are associated with the density and Young's modulus of each element in the designable region [2]. These design variables will have a value that is between zero and one. The element will have its normal mass and stiffness when the design variable has a value of one. A design variable equal to zero indicates that the element has no mass or stiffness.

To give a simple illustration of topology optimisation, consider the block of material, containing 3-D solid elements, shown in Figure 2.9. This example was acquired from the GENESIS User Manual [15]. The block is supported at the four lower corners and a concentrated load is applied at the middle point of the bottom plane. The block consists of 4000 hexagon elements which is the designable region. The objective function is to minimise the strain energy subject to a $25 \%$ mass constraint. In other words, the optimiser will make the structure as stiff as possible while keeping only $25 \%$ of the original mass.

Topology optimisation was applied to the block of material and the results are shown in Table 2.3 and Figure 2.10. The strain energy decreased by $97 \%$ or $628.8 \mathrm{MJ}$ in 9 design cycles. The final design has only $25 \%$ of the material as compared to the initial design. Figure 2.10(a) shows the final design, where 


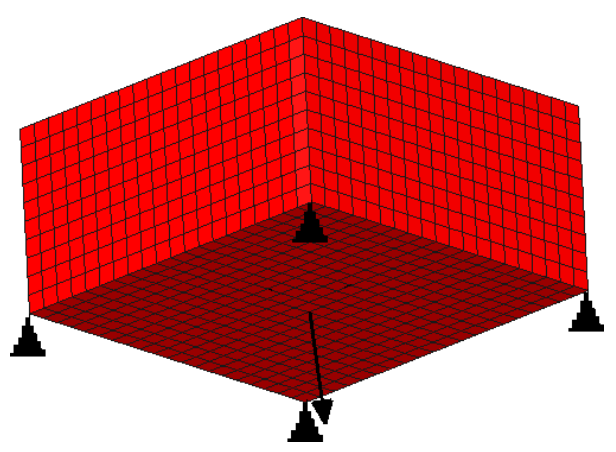

Figure 2.9: Rectangular block of material [15]

Figure 2.10(b) illustrates the material that was kept (green) and the material that was removed (blue) during the topology optimisation. This example shows that structural optimisation can be used for concept generation.

Table 2.3: Strain energy results for the topology optimisation

\begin{tabular}{lc}
\hline Parameter & Value \\
\hline \hline Number of design cycles & 9 \\
Original strain energy (MJ) & 647.3 \\
Optimised strain energy (MJ) & 18.5 \\
\hline
\end{tabular}

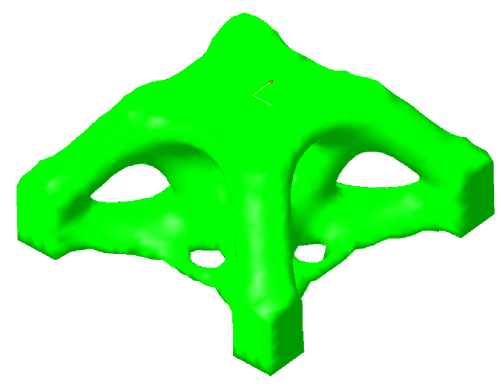

(a) The material that is left after topology optimisation

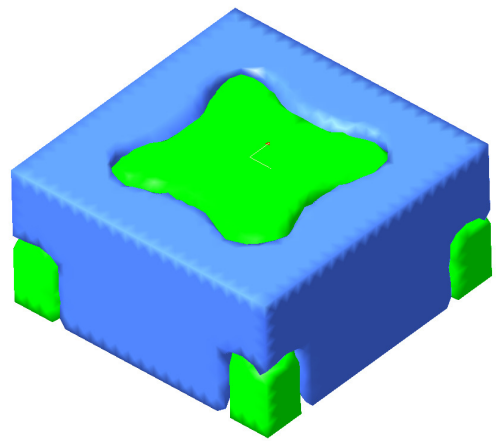

(b) The material that was kept (green) and the material that was removed (blue)

Figure 2.10: Results of the topology optimisation

\subsubsection{Topometry optimisation}

Topometry optimisation [14] provides a specialised implementation of size optimisation. It allows for the independent optimisation of all elements on an element 
by element level, whereas size optimisation is applied to a group of elements which is associated to a property data entry. Therefore topometry optimisation is size optimisation on an element level instead of a property level.

\subsubsection{Topography optimisation}

Topography optimisation [28] is a specialised implementation of shape optimisation. It is mostly used to increase the stiffness of shell or composite elements by adding curvature to a structure. For example the design of bead patterns on car floor panels. The grids of the designable region are allowed to move either normal to their original locations or in a specified direction. Topography optimisation requires the creation of specialised perturbation vectors (referred to as topography perturbation vectors) and their associated design variables. 


\section{Chapter 3}

\section{The Karoo Array Telescope}

The KAT will be used as an example to illustrate different structural optimisation applications. Figure 3.1(a) shows the KAT prototype during construction, while Figure 3.1(b) shows the completed prototype. Note that structural optimisation will be applied to this finalised prototype design, after it went through the entire design process. The prototype was designed without the use of any formal optimisation techniques. In this chapter the background and structural layout of the KAT is discussed and the design requirements are presented.

\subsection{Background}

The KAT is part of the MeerKAT project which is a predecessor of the Square Kilometer Array (SKA). The SKA will be the largest radio telescope ever built, with planned construction between 2014 and 2020. The proposed design for the SKA will consist of about 4500 antennas with a diameter of 10-15 meters each. The resulting combined area of all the antennas will be roughly a square kilometer. The SKA will be a radio telescope which collects electromagnetic signals omitted by far off planets and stars and converts these signals into images. It is an international project which will be funded by various countries and organisations. At this stage the bid is between South Africa and Australia to build the SKA on their home soil.

The MeerKAT will be a smaller version of the SKA project and was launched by the South African Government to demonstrate to the international community that the country can successfully undertake such a large scale project. The MeerKAT project will consist of an array of about sixty dishes. The diameter of the dishes will be 12 meters resulting in a collecting area of roughly $1 \%$, compared to that of the SKA. The MeerKAT will however still be a powerful scientific 
tool for studying the universe. It will be built near the proposed site for the SKA in the Northern Karoo, South Africa but the control station will be stationed in Cape Town, South Africa.

The MeerKAT project will be completed in three phases. During phase one a one-dish prototype (KAT) was designed and built. Phase two will consist of a seven-dish engineering test bed and scientific instrument called the KAT-7. During the final stage the MeerKAT will be built which will consist of the full array of about sixty dishes.

For both the SKA and KAT projects, reducing the cost of each antenna can lead to major cost savings. At this stage the cost for building the KAT antenna is too high and a means of further cost reduction is required. One technique that is investigated is the use of numerical design optimisation to minimise weight subject to constraints on stress, displacement, natural frequency, dish surface accuracy, etc. The goal is to find the lightest structure that will satisfy the design requirements. In this study the KAT antenna will be used as an example to show the different uses of structural optimisation with the goal of reducing cost.

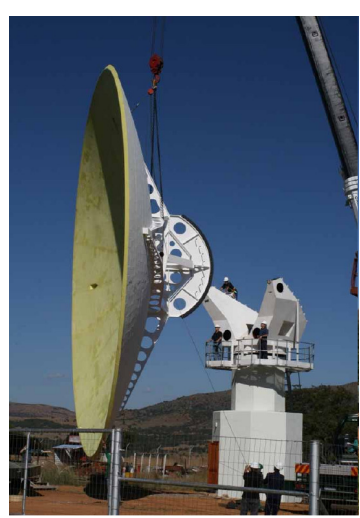

(a) The KAT during construction

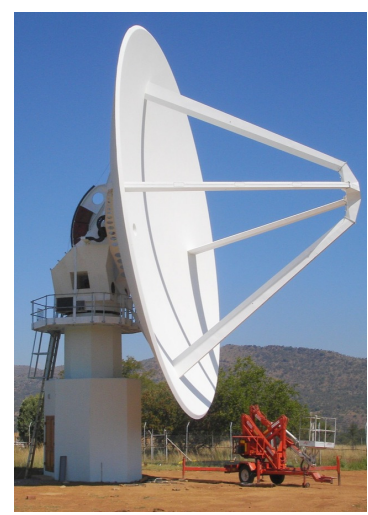

(b) The assembled KAT

Figure 3.1: The prototype KAT antenna

\subsection{The structural design}

The KAT antenna can be divided into five major components (see Figure 3.2): pedestal, yoke, backing structure, dish and Focal Plane Array (FPA) arms. The pedestal is a stiff concrete structure. An azimuth ring gear is placed on top of the pedestal and is responsible for rotation about the vertical axis. The yoke, attached to the azimuth ring gear, is a steel plate structure that provides rotation 
about the horizontal axis. The steel backing structure with a steel sector gear and counter weights are situated at the back of the dish. The backing structure rotates on two bearings in the yoke to provide the dish with a pitching capability. The dish is a composite quasi-honeycomb sandwich structure. The FPA arms are located at the front of the dish and supports the FPA unit. The FPA arms are thin steel tubular structures.

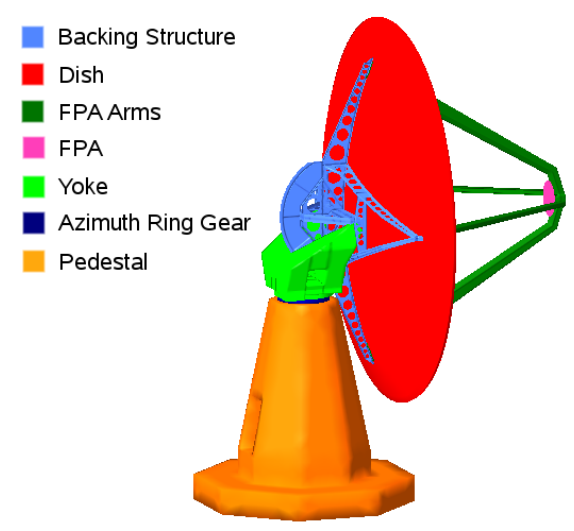

Figure 3.2: Components of the KAT

\subsection{Design specifications}

The goal of the present study is to provide the cheapest KAT antenna that still meets the basic design requirements for a useful scientific instrument. The design specifications [29] that have to be considered to obtain an acceptable KAT design are summarised below:

- The antenna must have a $15 \mathrm{~m}$ diameter prime focus dish.

- The dish surface-to-focus-point error (see an explanation below) should be $4 \mathrm{~mm}$ root mean square (RMS) with a target of $2 \mathrm{~mm}$ RMS.

- The maximum dish surface-to-focus-point error (see an explanation below) should be $6 \mathrm{~mm}$ with a target of $3 \mathrm{~mm}$.

- The FPA should have a maximum YZ-plane displacement of $\pm 10 \mathrm{~mm}$.

- The FPA should have a maximum X-displacement of $\pm 10 \mathrm{~mm}$.

- The maximum FPA rotation around the Y- and Z-axes should be $0.1^{\circ}$. 
- The maximum FPA rotation around the $\mathrm{X}$-axis should be $0.02^{\circ}$.

- The lowest natural frequency of the structure should be larger than $3 \mathrm{~Hz}$.

- Wind, gravity and thermal loads should be taken into account.

- The KAT should be able to operate in the normal operating environment and only survive the survival conditions (see Table 3.1).

Table 3.1: Normal and survival environments [29]

\begin{tabular}{lcc}
\hline Parameter & $\begin{array}{c}\text { Normal operation } \\
\text { environment }\end{array}$ & $\begin{array}{c}\text { Survival } \\
\text { environment }\end{array}$ \\
\hline \hline Minimum temperature $\left({ }^{\circ} \mathrm{C}\right)$ & -5 & -20 \\
Maximum temperature $\left({ }^{\circ} \mathrm{C}\right)$ & 40 & 60 \\
Maximum wind velocity $(\mathrm{km} / \mathrm{h})$ & 36 & 160 \\
\hline
\end{tabular}

For the requirements, both the dish surface-to-focus-point errors and the FPA displacements and rotations are measured relative to the best fit parabola, which are obtained from (3.3.1) [29]. In (3.3.1) $f_{d}$ is the focal distance which is equal to $7.5 \mathrm{~m}$.

$$
x=\frac{\left(y^{2}+z^{2}\right)}{4 f_{d}}
$$

The dish surface-to-focus-point error is a parameter that indicates the error of the dish surface to the focus point between the loaded and unloaded dish. It is the difference in the focal distances between the actual grid displacements and an idealised paraboloid, which is fitted to the actual grid displacements. From here on the dish surface-to-focus-point error will be referred to as the dish surface error to make it easier for the reader.

The FPA displacement is actually the relative displacement between the FPA and the focus point of the fitted paraboloid (like-wise for the FPA rotations). These parameters indicate if the focus point of the dish is located on the FPA after the dish has been loaded. If this is not the case, then the FPA will not detect the signal reflected from the dish. 


\section{Chapter 4}

\section{The Finite element model}

The KAT design team generated an ANSYS finite element model of the KAT antenna during the design process of the prototype. For this study, the ANSYS model was converted to MSC.NASTRAN format. The MSC.NASTRAN format was required to allow the use of GENESIS for both analysis and optimisation of the structure, since GENESIS is IO compatible with MSC.NASTRAN. GENESIS is a general purpose finite element analysis code that was developed from the start to be an advanced structural optimisation code.

The conversion and modification of the finite element model is discussed in this chapter. A comparison of the original ANSYS model and the altered MSC.NASTRAN model will be provided.

Note that the main focus of this study is not to find a GENESIS finite element model that exactly reproduces the results of the ANSYS finite element model. Instead, the goal is to find a realistic model, which can be used to illustrate structural optimisation applications. The GENESIS model should be representative of the KAT prototype in order for the improvements on the model to accurately portray the possible improvements on the prototype. The optimisation results will be compared relative to the GENESIS results of the prototype. The ANSYS results will only be used to ensure that the GENESIS finite element model is a realistic model of the prototype.

\subsection{The ANSYS finite element model}

The original ANSYS finite element model [29] is shown in Figure 4.1. The yoke and backing structure were modelled using shell elements. The quasi-honeycomb core of the dish is modeled with 3D solid elements, with the rear and front skins as composite shell elements. The FPA arms were modeled as shell elements with 
the FPA itself as a $1.5 \mathrm{~m}$ diameter disk with a mass of $300 \mathrm{~kg}$. The model includes gravity, temperature and wind loads.

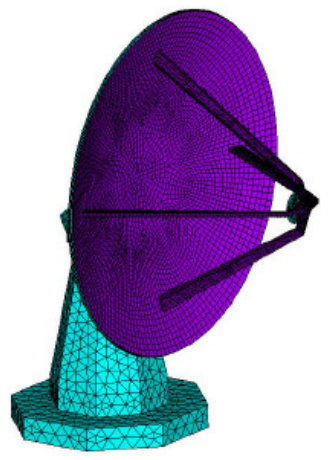

(a) Front view

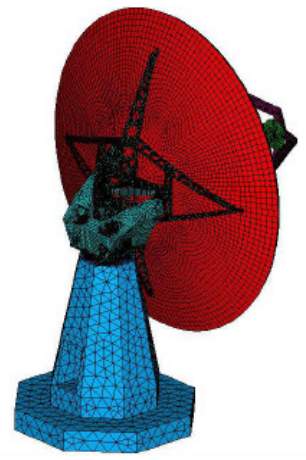

(b) Back view

Figure 4.1: The ANSYS finite element model of the KAT [29]

\subsection{Loadcases}

In reality, the KAT antenna is subject to an infinite number of loadcases. The ANSYS finite element model accounted for these by incorporating various loads including temperature, wind and gravity loads [29]. For some loadcases the dish were modelled at different pitch and azimuth angles while in other loadcases the wind direction was altered. The situation of an emergency stop was also investigated.

Since the aim of this project is not to generate a final design for the KAT, but to give suggestions on saving mass, only the most extreme loadcases will be considered in the GENESIS finite element model. One wind load with the wind blowing from the front of the antenna was considered. The most extreme temperature loads from the ANSYS model were included in the GENESIS finite element model as well as a gravity load. The KAT antenna was only analysed at zero pitch and azimuth, because this configuration produces the maximum wind force on the dish. The emergency stop load was omitted from the finite element model as the theory behind it was outside the scope of this thesis. The list of loads that were included in the finite element model are as follow:

1. Gravity load.

2. Wind at a speed of $36 \mathrm{~km} / \mathrm{h}$ (normal operation conditions), from the front of the antenna. 
3. Wind at a speed of $160 \mathrm{~km} / \mathrm{h}$ (survival conditions), from the front of the antenna.

4. Temperature load of $-5{ }^{\circ} \mathrm{C}$ on the whole structure from a $20^{\circ} \mathrm{C}$ reference.

5. Temperature load of $60{ }^{\circ} \mathrm{C}$ on the front of the dish with the rest of the structure at $40{ }^{\circ} \mathrm{C}$ from a $20{ }^{\circ} \mathrm{C}$ reference.

6. Temperature load of $60{ }^{\circ} \mathrm{C}$ on the rear of the dish with the rest of the structure at $40{ }^{\circ} \mathrm{C}$ from a $20{ }^{\circ} \mathrm{C}$ reference.

7. Temperature load of $60{ }^{\circ} \mathrm{C}$ on the top of the dish with the rest of the structure at $40{ }^{\circ} \mathrm{C}$ from a $20{ }^{\circ} \mathrm{C}$ reference.

8. Normal modes analysis.

As the above loads do not occur on their own, combinations of the loads are considered during the design process. Table 4.1 shows the loadcases that were used:

Table 4.1: Loadcases in the finite element model

\begin{tabular}{ccccc}
\hline $\begin{array}{c}\text { Loadcase } \\
\text { number }\end{array}$ & Gravity & Wind & Temp & $\begin{array}{c}\text { Normal } \\
\text { Modes }\end{array}$ \\
\hline \hline A & 1 & 2 & 4 & - \\
B & 1 & 2 & 5 & - \\
C & 1 & 2 & 6 & - \\
D & 1 & 2 & 7 & - \\
E & - & - & - & 8 \\
F & - & 3 & - & - \\
\hline
\end{tabular}

The loadcases can be described as:

A. A typical cold winter's night with the wind blowing from the front (normal operation conditions).

B. A typical hot summer's day with both the sun shining and the wind blowing from the front (normal operation conditions).

C. A typical hot summer's day with the sun shining from the back and the wind blowing from the front (normal operation conditions).

D. A typical hot summer's day with the sun shining from the top and the wind blowing from the front (normal operation conditions). 
E. Normal modes analysis to obtain the natural frequencies of the structure.

F. A typical survival condition with a strong wind blowing from the front.

The reason for only having a wind load in loadcase $\mathrm{F}$ is that the antenna will be in its survival operating condition where it is only necessary to evaluate the stresses to make sure it does not fail. The antenna does not have to be operational in these conditions and it is unnecessary to consider the temperature and gravity loads since it mainly influences the accuracy of the dish.

Loadcases B, C and D have the same loads compared to these loadcases from the ANSYS model. The only difference is that the dish is at zero azimuth compared to the $90^{\circ}$ azimuth dish of the ANSYS model. Loadcase A is the only loadcase that is identical for both models.

Note that the mechanical properties of steel change by an increase in temperature, but its properties only starts to deteriorate at temperatures of between $200{ }^{\circ} \mathrm{C}$ and $300{ }^{\circ} \mathrm{C}$ [30]. In this study the highest temperature load is $60{ }^{\circ} \mathrm{C}$, which is far from the critical temperature. Therefore steel's properties were kept constant during the study.

\subsection{Conversion of the finite element model}

The finite element model that was received from the KAT design team was created in ANSYS. As GENESIS is IO compatible with MSC.NASTRAN, the ANSYS finite element model was converted to MSC.NASTRAN format. The model was converted by Esteq (the MSC agents in South Africa). After conversion, a few defects were identified in the MSC.NASTRAN finite element model. The defects were a result of the conversion process, during which information were lost.

After conversion, the finite element model only consisted of element and grid data. Material-, property-, load- and support data were still needed to create a valid finite element model that could be used for analysis and optimisation. In addition, the shell elements in the model were eight noded quadrilateral (CQUAD8) and six noded triangular (CTRIA6), quadratic elements, while GENESIS can only deal with four noded quadrilateral (CQUAD4) and three noded triangular (CTRIA3), linear elements. Finally, one of the components had an incomplete mesh while some parts of the model were disconnected. These defects were rectified, as explained in the following sections. 


\subsubsection{Load data}

The material-, property-, load- and support data were obtained from the KAT design team [29] and added to the model. Unfortunately only a small amount of data were acquired for the temperature loads, while no data were obtained for the wind load.

An assumption was made that wind results in a pressure load on the dish, directly from the front. The pressure was calculated using (4.3.1) [31] and dividing both sides by the area, resulting in (4.3.2) ( $q$ is given by (4.3.3) [31]).

$$
\begin{gathered}
F=q C_{d} A \\
P=q C_{d} \\
q=\frac{1}{2} \rho v^{2}
\end{gathered}
$$

For simplicity $C_{d}$ was assumed equal to one and the density of air equal to $1.168 \mathrm{~kg} / \mathrm{m}^{3}$ (calculated at $25^{\circ} \mathrm{C}$ and $100 \mathrm{kPa}$ ). According to literature $C_{d}$ should be between 1.1 and 1.4 [32]. However, a wind tunnel test or Computational Fluids Dynamics (CFD) analysis is necessary to determine a more accurate value. For a wind speed of $36 \mathrm{~km} / \mathrm{h}$ and $160 \mathrm{~km} / \mathrm{h}$ (as summarised in Table 3.1), the calculated pressure on the dish was $58.4 \mathrm{~Pa}$ and $1151.3 \mathrm{~Pa}$ respectively. These pressures were applied to each finite element on the front part of the dish. The results obtained from this approach correlated well with the results obtained from the ANSYS model.

No data were available for the temperature distribution on each grid point. Only pictures of the temperature distributions on the antenna were available for load 5, load 6 and load 7 (refer to Section 4.2 for an explanation of each load). These pictures were studied and similar temperature distributions were applied to the GENESIS model. Figure 4.2 shows a comparison between the temperature distributions for the two models (Table 4.2 shows the temperature values represented by the colours).

In comparison, the temperature distributions for the GENESIS and ANSYS models are very similar as displayed by Figure 4.2. There is only a small difference in the temperature distribution on the dish rear for load 7 (see Figures $4.2(\mathrm{~g})$ and $4.2(\mathrm{c}))$. Another small variation between the temperature distributions for the two models are that the bottom of the ANSYS dish is at $57^{\circ} \mathrm{C}$ while the GENESIS dish is at $63{ }^{\circ} \mathrm{C}$, for load 7 (see Figures $4.2(\mathrm{~h})$ and $4.2(\mathrm{~d})$ ). 


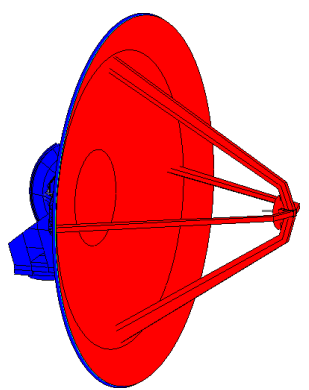

(a) Load 5

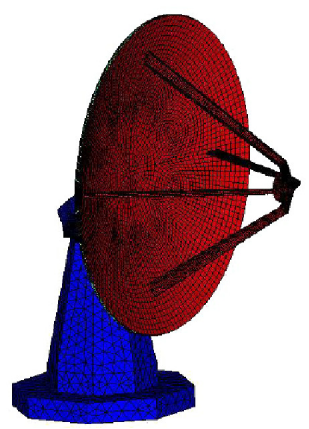

(e) Load 5

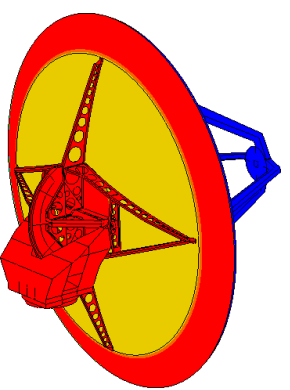

(b) Load 6

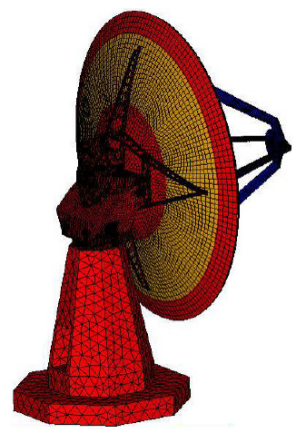

(f) Load 6

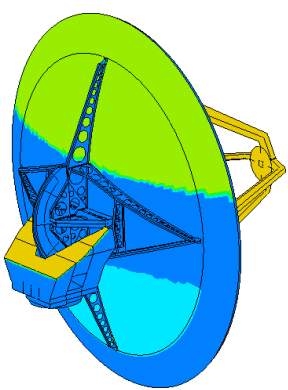

(c) Load 7 (rear)

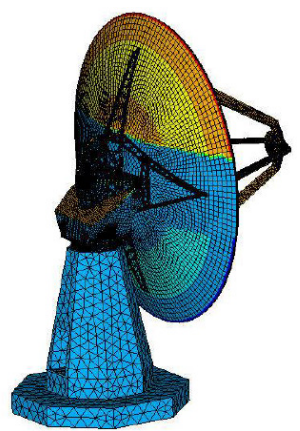

(g) Load 7 (rear)

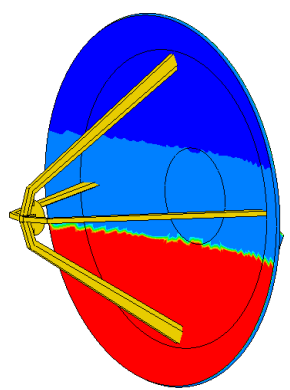

(d) Load 7 (front)

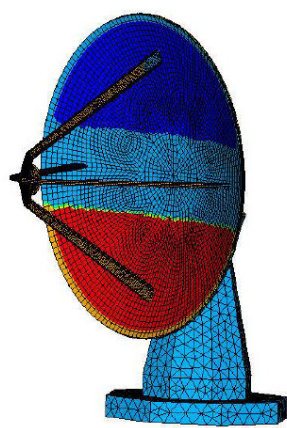

(h) Load 7 (front)

Figure 4.2: A comparison of the temperature loads between the GENESIS- (top) and ANSYS (bottom) [29] models

Table 4.2: The temperature values of the temperature loads depicted in Figure 4.2

\begin{tabular}{ccccccccc}
\hline $\begin{array}{c}\text { Load } \\
\text { no. }\end{array}$ & Model & $\begin{array}{c}\text { Dark } \\
\text { blue }\end{array}$ & $\begin{array}{c}\text { Light } \\
\text { blue }\end{array}$ & Red & $\begin{array}{c}\text { Dark } \\
\text { yellow }\end{array}$ & $\begin{array}{c}\text { Light } \\
\text { yellow }\end{array}$ & Green & Cyan \\
\hline \hline 5 & GEN. $\left({ }^{\circ} \mathrm{C}\right)$ & 40 & - & 60 & - & - & - & - \\
& ANS. $\left({ }^{\circ} \mathrm{C}\right)$ & 40 & - & 60 & - & - & - & - \\
\hline 6 & GEN. $\left({ }^{\circ} \mathrm{C}\right)$ & 40 & - & 60 & 56 & - & - & - \\
& ANS. $\left({ }^{\circ} \mathrm{C}\right)$ & 40 & - & 60 & 56 & - & - & - \\
\hline 7 & GEN. $\left({ }^{\circ} \mathrm{C}\right)$ & 36 & 42 & 63 & 57 & - & 55 & 45 \\
& ANS. $\left({ }^{\circ} \mathrm{C}\right)$ & 36 & 42 & 63 & 57 & 54 & - & 45 \\
\hline
\end{tabular}

These differences are small enough to result in similar results for both models.

There was not a picture available for load 4 . The only information to work with, was that the whole antenna was loaded with a $-5^{\circ} \mathrm{C}$ load which was applied to the GENESIS model. 


\subsubsection{Incomplete mesh}

The azimuth ring gear, which connects the pedestal with the yoke, had an incomplete mesh after the conversion (see Figure 4.3). After various attempts of re-meshing the azimuth ring gear it was impossible to match the nodes on the lower surface of the ring with the nodes on the upper surface of the pedestal.

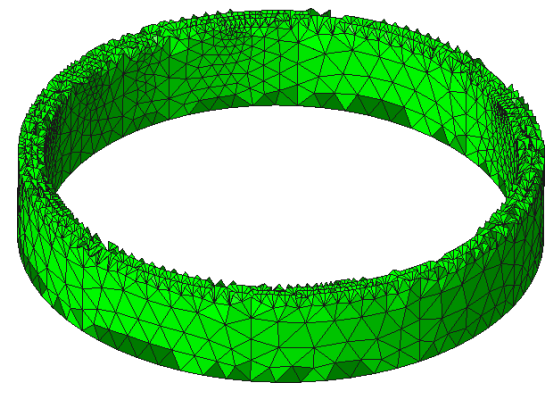

Figure 4.3: Incomplete mesh of the azimuth ring gear

An assumption was made by omitting the pedestal from the finite element model as the nodes at the interface did not match. The pedestal is a concrete structure which is very stiff compared to the rest of the structure. The exclusion of the pedestal from the model will have a small effect on the rest of the structure. This was confirmed by analysing the pedestal with the loads it experiences which are wind, gravity and three temperature loads (for a discussion of the loads induced on the antenna, see Section 4.2). Note that the wind load was modelled as a point force and moment on top of the pedestal. Table 4.3 displays the minimum and maximum displacements of the pedestal.

Table 4.3: Displacements of the pedestal for various loads

\begin{tabular}{lccc}
\hline Load & $\mathrm{X}(\mathrm{mm})$ & $\mathrm{Y}(\mathrm{mm})$ & $\mathrm{Z}(\mathrm{mm})$ \\
\hline \hline Gravity & 0 & 0 & -0.34 \\
Temp: $60{ }^{\circ} \mathrm{C}$ & \pm 1.1 & \pm 1.1 & 3.4 \\
Temp: $40{ }^{\circ} \mathrm{C}$ & \pm 0.7 & \pm 0.7 & 2.3 \\
Temp: $-5{ }^{\circ} \mathrm{C}$ & 0 & 0 & -0.29 \\
Wind: $36 \mathrm{~km} / \mathrm{h}$ & 0.2 & 0 & 0 \\
\hline
\end{tabular}

Only the maximum $Z$-displacement is given in Table 4.3 (the values smaller than $1.0 \mathrm{E}^{-1}$ are noted as zero), since the minimum value will be zero or very near to zero. All displacements are small, except for the $Z$-displacements of the $60^{\circ} \mathrm{C}$ and $40{ }^{\circ} \mathrm{C}$ loads, which occur at the top of the pedestal (as shown by Figure 4.4 
for the $60{ }^{\circ} \mathrm{C}$ temperature load). However, these loads will result in a uniform up- or down movement of the dish and as a result will have a small influence on the structural- and non-structural responses considered during the optimisation. The non-structural responses are based on relative displacements between the FPA and dish. Therefore it is a reasonable assumption to omit the pedestal from the GENESIS finite element model.

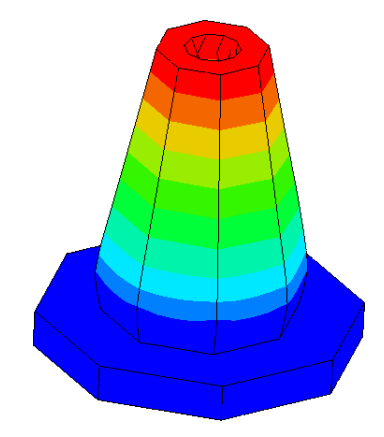

Figure 4.4: Displacements on the pedestal due to a $60{ }^{\circ} \mathrm{C}$ temperature load $($ red $=3.4 \mathrm{~mm}$ and blue $=0 \mathrm{~mm})$

The boundary conditions of the ANSYS model were located at the bottom of the pedestal. As the pedestal is no longer part of the model, the boundary conditions had to be applied somewhere else. The logical spot was to apply them at the bottom of the azimuth ring gear. All six degrees of freedom were constrained. This is a reasonable assumption since the concrete pedestal is very stiff and experience small displacements.

\subsubsection{Disconnected components}

Some parts of the backing structure were not connected, while the dish and FPA arms were not connected to the backing structure at all. These connections were lost during the conversion of the model and it was uncertain what type of elements ANSYS used for the connections. RBE2 elements, which are rigid and experience no deformation when loaded, were used to connect these components. The RBE2 elements were set up in such a way that the six degrees-of-freedom of the independent nodes, are transported to the dependent nodes. The rigid elements which connect the dish with the backing structure as well as the connections in the backing structure itself, can be seen in Figures 4.5(a) and 4.5(b) (rigid elements shown in blue and encircled in Figure 4.5(a)).

The steel sector gear should be connected to the yoke to keep the dish at the correct pitch angle. RBE2 elements were also inserted to connect the yoke 


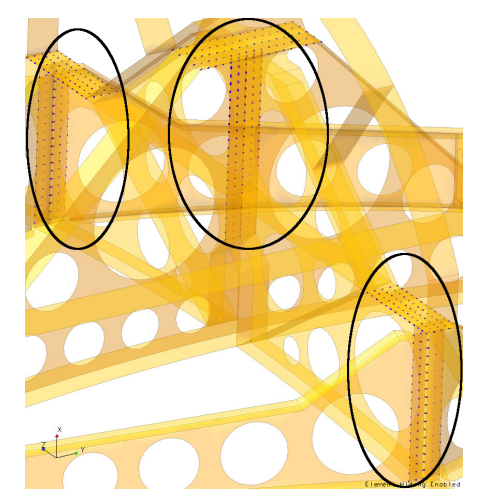

(a) Rigid elements (encircled) connecting the backing structure

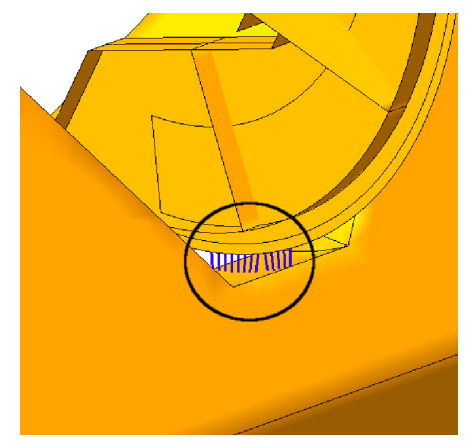

(c) Rigid elements (encircled) connecting the yoke with the sector gear

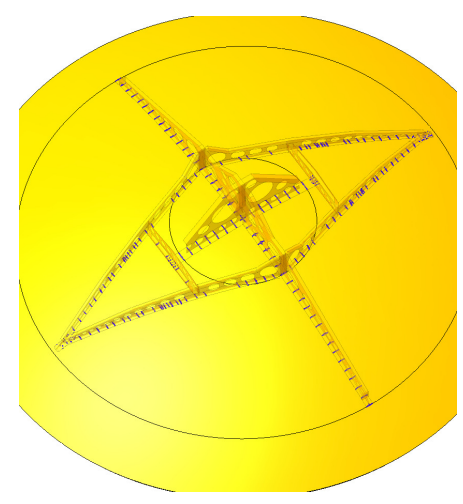

(b) Rigid elements connecting the backing structure with the dish

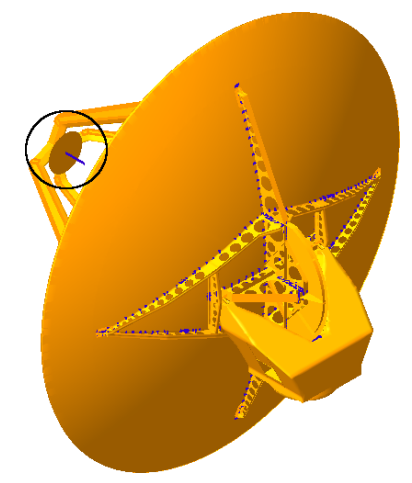

(d) Rigid element (encircled) connecting the focus point with the FPA

Figure 4.5: Rigid elements inserted into the finite element model

with the steel sector gear (see encircled part in Figure 4.5(c), with rigid elements shown in blue).

A problem that was identified in the ANSYS model is that the FPA is not at the focus point of the unloaded structure. There was a $830 \mathrm{~mm}$ offset in the $X$-direction (along the optical axis). This is quite important for the calculation of the dish surface accuracy (this is explained in Section 3.3). To rectify this offset, a grid point was created at the focus point of the unloaded dish and it was connected to the FPA with a rigid element (see encircled part in Figure 4.5(d)).

The use of rigid elements can be problematic with thermal loads, because the rigid elements can not expand with the adjacent structure. This could result in artificially high stresses and/or induce errors in deflections (high or low). The inaccurate stresses were accounted for by excluding all shell elements, which are connected to a rigid element, from the stress constraints. Forty percent of the rigid elements were removed from the finite element model to determine the influence of the rigid elements on the antenna's displacements during thermal 
loads. It resulted in a small change (less than a millimetre) in the displacements of the FPA and the antenna.

\subsubsection{Reduction in shell element order}

All quadratic shell elements were replaced with linear shell elements by means of a Python script, which was developed by the author. The Python program searched through the MSC.NASTRAN input data for all CQUAD8 and CTRIA6 elements. The CQUAD8 and CTRIA6 entries were changed to CQUAD4 and CTRIA3 elements, during which the midside nodes were deleted while the corner nodes were retained. A new file was written containing only the CQUAD4 and CTRIA3 elements with their respective nodes. The rest of the MSC.NASTRAN input data was left unchanged.

The reduction from quadratic- to linear elements could lead to a loss of accuracy. To verify that the mesh has converged, another finite element model was created by dividing all the non-rigid finite elements into four elements, creating a finer mesh. This mesh refinement increased the degrees of freedom from 400000 to 2000000 .

Table 4.4 displays the difference in the displacements (magnitude) and the natural frequencies for the GENESIS model with the original- and the refined mesh (the values smaller than $1.0 \mathrm{E}^{-1}$ are noted as zero). The refined mesh leads to a small increase in the displacements with load 6 experiencing the largest increase of $16 \%$ (printed in bold). The natural frequencies for the two cases compare very well where a small decrease in the natural frequency for each mode shape is noticed. The largest decrease of $6 \%$ is seen with load 7 (printed in bold). The modes shapes remained the same. The difference in displacements and natural frequencies between the two cases can be ascribed in part to the fact that the number of rigid elements remained constant during the mesh-refinement. For a better comparison, the number of rigid elements should be increased by four times. This was not done as the insertion of rigid elements is a time consuming task. 
Table 4.4: The magnitude displacement and natural frequency variances between the GENESIS finite element model with the original- and refined mesh

\begin{tabular}{|c|cc|cc|c|cc|}
\hline \multirow{2}{*}{$\begin{array}{c}\text { Load } \\
\text { no. }\end{array}$} & \multicolumn{2}{|c|}{$\triangle$ Disp. $(\mathrm{mm})$} & \multicolumn{2}{c|}{$\triangle$ Disp. $(\%)$} & Mode & $\begin{array}{c}\triangle \text { Natural } \\
\text { frequencies }\end{array}$ & $\begin{array}{c}\triangle \text { Natural } \\
\text { frequencies }(\%)\end{array}$ \\
\hline \hline 1 & 0 & 1.2 & 0 & $9 \%$ & 1 & -0.1 & $-3 \%$ \\
2 & 0 & 0.1 & 0 & $11 \%$ & 2 & -0.1 & $-3 \%$ \\
4 & 0 & 0.2 & 0 & $4 \%$ & 3 & -0.3 & $-\mathbf{6} \%$ \\
5 & 0 & -0.1 & 0 & $-1 \%$ & 4 & -0.1 & $-2 \%$ \\
6 & 0 & $\mathbf{1 . 0}$ & 0 & $\mathbf{1 6 \%}$ & 5 & -0.1 & $-2 \%$ \\
7 & 0 & 0.8 & 0 & $8 \%$ & & & \\
\hline
\end{tabular}

Consider Figure 4.6, which displays the Von Mises shell element stress for the original- and refined mesh under gravity loading (the other loads gave similar results). The scale of both plots were synchronised to allow for easy comparison. Figures 4.6(a) and 4.6(b) are almost identical indicating that the stresses in the original mesh is converged and that a finer mesh is not necessary. The maximum stresses for both cases differ as a result of stress concentrations. This is expected as an increase in mesh quality will lead to an increase in stress at the stress concentrations.

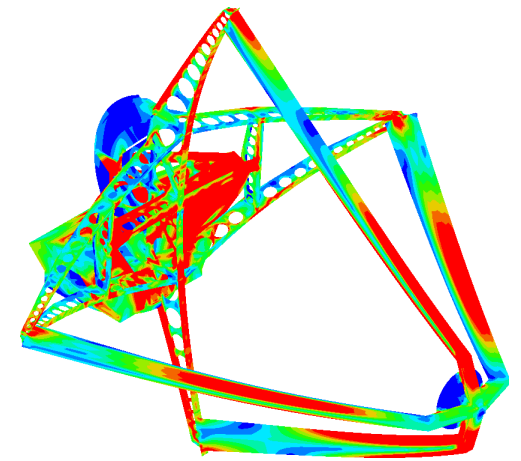

(a) Original mesh: red $=8 \mathrm{MPa}$, green $=4 \mathrm{MPa}$, blue $=31 \mathrm{kPa}$

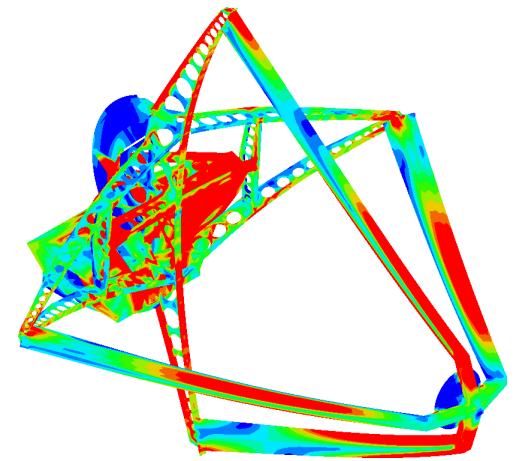

(b) Refined mesh: red $=8 \mathrm{MPa}$, green $=4 \mathrm{MPa}$, blue $=16 \mathrm{kPa}$

Figure 4.6: Shell element Von Mises stress for the GENESIS model with the originaland refined mesh under gravity loading

The connection of shell elements with rigid- or bar elements will result in higher stresses at the connections. This will cause stress concentrations in the finite element model which are actually not present in the physical structure. A connection of a shell element with a bar element occur at the connection of the yoke with the backing structure, while the connections of shell elements with rigid elements can be seen in Section 4.3.3. 
The stress constraint bounds that were used during the optimisation were not set equal to the maximum stress which occur at a connection of a shell element with a bar- or rigid element. The elements at these connections were excluded from the constraints as they do not give an accurate representation of the stress distribution in the structure. If these elements were included in the constraints, then the optimiser will strengthen these parts, because of the stress concentrations. This is not desireable as the wrong parts in the structure will be strengthened.

\subsection{Finite element model comparison}

The final finite element model of the KAT antenna used in this study is shown in Figures 4.7(a) and 4.7(b). The model contains approximately 80000 grid points, 100000 elements and \pm 400000 degrees of freedom.

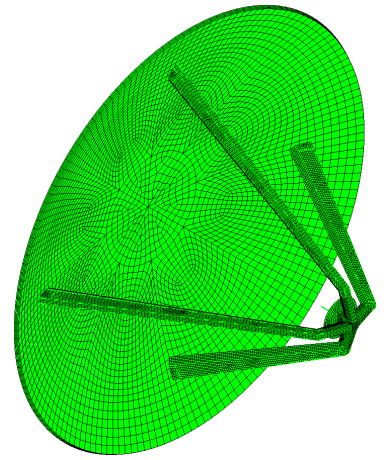

(a) Front view

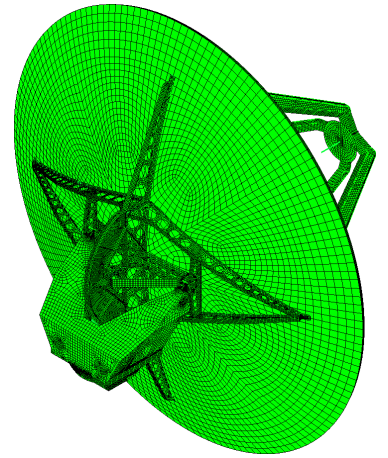

(b) Back view

Figure 4.7: KAT finite element model

A modal test on the prototype could have helped in validating the GENESIS model. This was not a viable option since a modal test is expensive. The last resort was to compare the GENESIS model with the ANSYS model, even though it is not known if the ANSYS model is perfect (no single model can be accepted as correct). As the two finite element models are not exactly the same, one would expect some variance in the results.

A comparison of the minimum and maximum displacements between the GENESIS and ANSYS models (for loads 1, 2, 4, 5, 6 and 7) are displayed in Table 4.5 (load 3 was not investigated since it will result in a similar displacement distribution than load 2, but the magnitude will only differ by a linear amount). The displacement ranges for the gravity and wind loads correlate well (no ANSYS results were available for the gravity $Y$-displacements or the wind 
$Y$ - and $Z$-displacements). The displacement contour plots for these two loads are also similar.

Table 4.5: Displacement ranges for the GENESIS and ANSYS [29] models

\begin{tabular}{|c|cc|cc|cc|cc|cc|cc|}
\hline \multirow{3}{*}{$\begin{array}{c}\text { Load } \\
\text { no. }\end{array}$} & \multicolumn{4}{|c|}{$\mathrm{X}(\mathrm{mm})$} & \multicolumn{4}{c|}{$\mathrm{Y}(\mathrm{mm})$} & \multicolumn{4}{c|}{ Z $(\mathrm{mm})$} \\
\cline { 2 - 13 } & \multicolumn{2}{|c|}{ GEN. } & \multicolumn{2}{|c|}{ ANS. } & \multicolumn{2}{c|}{ GEN. } & \multicolumn{2}{c|}{ ANS. } & \multicolumn{2}{c|}{ GEN. } & \multicolumn{2}{c|}{ ANS. } \\
\hline \hline 1 & $\mathbf{- 8 . 5}$ & 6.4 & -7.3 & 6.1 & - & - & - & - & -13 & 0.3 & -13 & 0.15 \\
2 & -0.2 & 0.9 & -0.2 & 0.9 & - & - & - & - & - & - & - & - \\
4 & $\mathbf{- 2 . 2}$ & $\mathbf{1 . 2}$ & $\mathbf{- 4}$ & $\mathbf{3 . 2}$ & -3.9 & 4.0 & -3.9 & 3.9 & $\mathbf{- 5 . 4}$ & $\mathbf{3 . 7}$ & $\mathbf{- 7 . 6}$ & $\mathbf{0 . 2}$ \\
5 & -2.3 & 7.5 & -2.9 & 8.7 & -6.4 & 6.3 & -6.8 & 6.8 & $\mathbf{- 6 . 7}$ & $\mathbf{7 . 9}$ & $\mathbf{- 3 . 8}$ & $\mathbf{9 . 7}$ \\
6 & $\mathbf{- 4 . 8}$ & $\mathbf{0 . 9}$ & $\mathbf{- 1 0}$ & $\mathbf{3 . 1}$ & -3.4 & 3.4 & -3.3 & 3.3 & $\mathbf{- 2 . 6}$ & $\mathbf{5 . 3}$ & $\mathbf{2 . 5}$ & $\mathbf{1 1}$ \\
7 & $\mathbf{- 4 . 4}$ & $\mathbf{8 . 7}$ & $\mathbf{- 4 . 5}$ & $\mathbf{5 . 4}$ & -4.7 & 4.7 & -4.8 & 4.8 & $\mathbf{- 7 . 4}$ & 3.6 & $\mathbf{- 4 . 7}$ & 4.9 \\
\hline
\end{tabular}

For the temperature loads, the $Y$-displacements, including the contour plots, are similar. There are however differences in the $X$ - and $Z$-displacements for the temperature loads. All displacements where the difference in the displacement ranges are greater than $1.5 \mathrm{~mm}$, have been printed in bold in Table 4.5. Load 4 and load 6 stand out as both their $X$ - and $Z$ ranges do not coincide.

The exclusion of the pedestal from the finite element model has an influence on the $Z$-displacements of the model as was shown in Table 4.3. The pedestal only has significant $Z$-displacements for the $60{ }^{\circ} \mathrm{C}$ and $40{ }^{\circ} \mathrm{C}$ loads. A $Z$-displacement at the top of the pedestal will cause the rest of the structure to displace with the same amount. Therefore the pedestal $Z$-displacements were added to the displacements of the temperature loads obtained from the GENESIS model, to compensate for the pedestal's omission from the model. $3.4 \mathrm{~mm}$ was added to load 6 as the pedestal will be loaded with $60{ }^{\circ} \mathrm{C}$ for this load. Similarly, $2.3 \mathrm{~mm}$ was added to loads 5 and 7 . These corrected $Z$-displacements can be seen in Table 4.6 (the $X$ - and $Y$-displacements were omitted as they remained unchanged after the corrections).

The ranges for the $Z$-displacements of loads 5 and 7 are similar after the correction. There are still considerable differences in the maximum $X$-displacement for load 7 and the $X$ - and $Z$-displacement ranges for loads 4 and 6 .

Consider, Figure 4.8 which displays the displacement contour plots for the problematic temperature loads of the GENESIS and ANSYS models. The contour plot of the $X$-displacement of load 7 is similar for both models. The only difference is that the $X$-displacement at the bottom of the dish is larger for the 
Table 4.6: Corrected displacement ranges for the GENESIS and ANSYS [29] models

\begin{tabular}{|c|c|c|c|c|}
\hline \multirow{3}{*}{$\begin{array}{c}\text { Load } \\
\text { no. }\end{array}$} & \multicolumn{4}{|c|}{$\mathrm{Z}(\mathrm{mm})$} \\
\hline & \multicolumn{2}{|c|}{ GENESIS } & \multicolumn{2}{|c|}{ ANSYS } \\
\hline & $\min$ & $\max$ & $\min$ & $\max$ \\
\hline 5 & -4.4 & 10.2 & -3.8 & 9.7 \\
\hline 6 & 0.8 & 8.7 & 2.5 & 11 \\
\hline 7 & -5.1 & 6.0 & -4.7 & 4.9 \\
\hline
\end{tabular}

GENESIS model. The reason for the difference is that the bottom of the ANSYS dish is at $57{ }^{\circ} \mathrm{C}$ while the GENESIS dish is at $63{ }^{\circ} \mathrm{C}$ (see Figure 4.2).

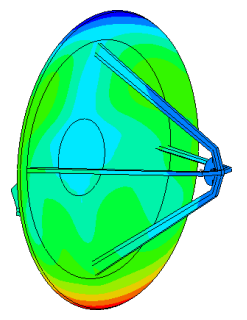

(a) Load 7: UX

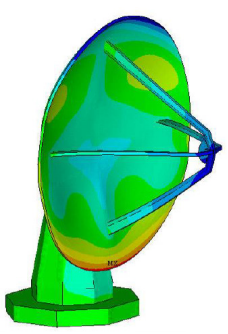

(f) Load 7: UX

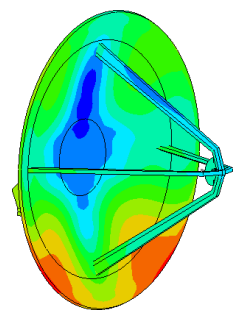

(b) Load 6: UX

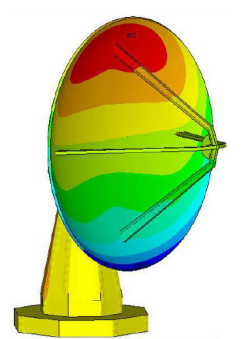

(g) Load 6: UX

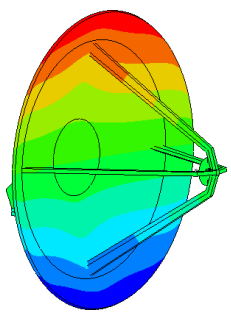

(c) Load 6: UZ

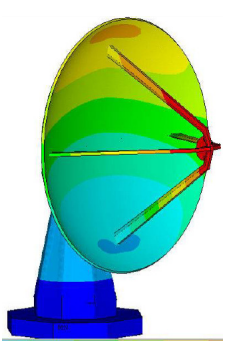

(h) Load 6: UZ

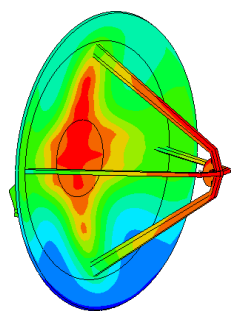

(d) Load 4: UX

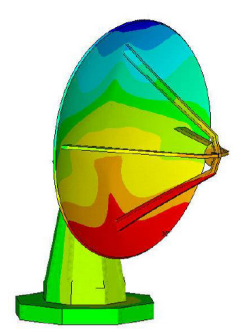

(i) Load 4: UX

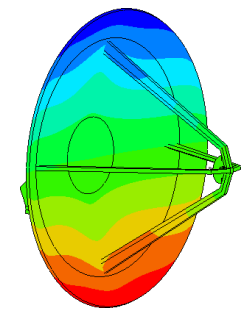

(e) Load 4: UZ

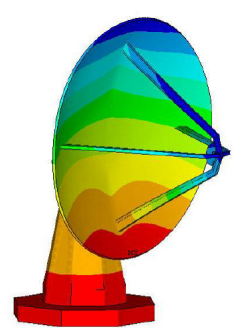

Figure 4.8: A comparison of displacements from temperature loads between the GENESIS- (top) and ANSYS (bottom) [29] models

The $X$-displacement contours of loads 4 and 6 do not have any similarity. The maximum and minimum displacements are not located on the same spots which explain the differences in the displacement ranges. The $Z$-displacement contours on the dish of loads 4 and 6 are similar and the maximum and minimum $Z$ displacements occur at the same spots. However, the maximum and minimum $Z$-displacements for the ANSYS antenna are on the FPA. For the GENESIS model the maximum and minimum $Z$-displacements are on the dish itself. This explain the differences between the two models for these cases.

Load 4 cools the structure, while load 6 heats it. Therefore the displacements of the two loads, should be the inverse of each other. This phenomenon is exactly 
what is observed with the GENESIS $X$ - and $Z$-displacement contours. This is not the case with the ANSYS model.

The available pictures of the temperature distributions were studied and the GENESIS loads were reproduced as closely as possible. As the temperature data on each grid point was unavailable it is impossible to duplicate the ANSYS temperature loads precisely. Therefore the differences in the two models' temperature load displacements can be ascribed to the loads not being absolutely identical.

A comparison between the modes shapes and natural frequencies for the GENESIS and ANSYS models are shown in Figure 4.9 and Table 4.7. The mode shapes for both models are the same except that the first- and second mode shapes have switched. The first two natural frequencies are quite close to each other, which could result in a switch between the first two modes. The first-, second- and fifth natural frequencies (shown in bold in Table 4.7) differ also by a small amount.

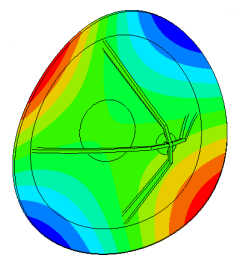

(a)

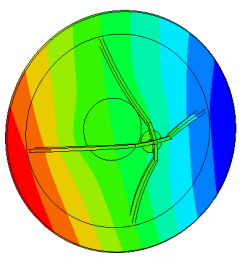

(b)

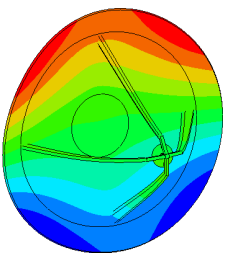

(c)

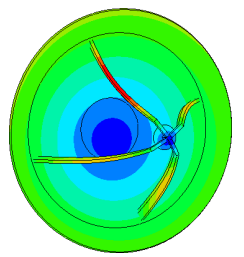

(d)

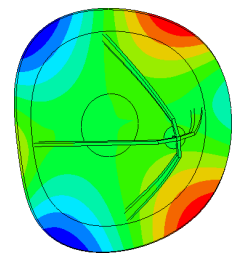

(e)

Mode $1(3.6 \mathrm{~Hz})$ Mode $2(3.7 \mathrm{~Hz}) \quad$ Mode $3(4.8 \mathrm{~Hz})$ Mode $4(5.2 \mathrm{~Hz})$ Mode $5(6.2 \mathrm{~Hz})$

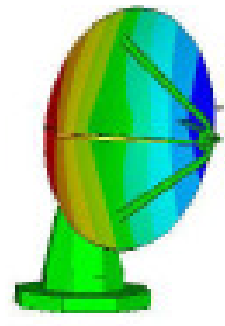

(f)

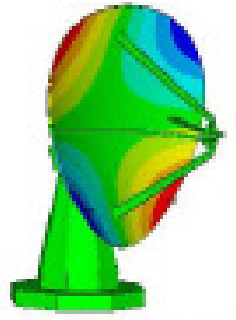

(g)

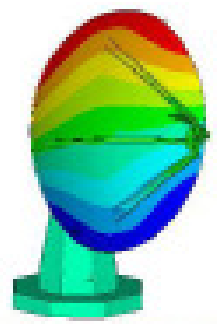

(h)

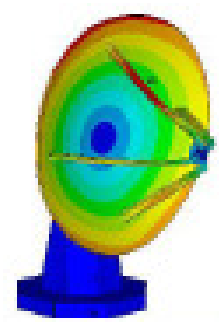

(i)

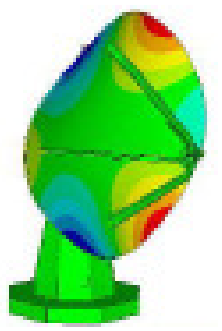

(j)

Mode $1(4.2 \mathrm{~Hz})$ Mode $2(4.3 \mathrm{~Hz})$ Mode $3(4.7 \mathrm{~Hz})$ Mode $4(5.5 \mathrm{~Hz})$ Mode $5(7.1 \mathrm{~Hz})$

Figure 4.9: A comparison of the first five natural modes between the GENESIS- (top) and ANSYS (bottom) [29] models

The results indicated that the GENESIS model is less stiff than the ANSYS model, since some of the GENESIS natural frequencies are smaller than the ANSYS natural frequencies. This result is counter-intuitive since removing the pedestal and using lower order elements will result in a stiffer model. It was investigated by removing some of the rigid elements that connects the dish with the backing structure from the GENESIS model and performing a normal modes 
Table 4.7: Natural frequencies for the GENESIS and ANSYS [29] models

\begin{tabular}{ccccc}
\hline \multirow{2}{*}{$\begin{array}{c}\text { Gode } \\
\text { number }\end{array}$} & $\begin{array}{c}\text { Frequency } \\
(\mathrm{Hz})\end{array}$ & $\begin{array}{c}\text { Mode } \\
\text { description* }\end{array}$ & $\begin{array}{c}\text { Frequency } \\
(\mathrm{Hz})\end{array}$ & $\begin{array}{c}\text { Mode } \\
\text { description* }\end{array}$ \\
\hline \hline 1 & $\mathbf{3 . 6}$ & 2 & $\mathbf{4 . 2}$ & 1 \\
2 & $\mathbf{3 . 7}$ & 1 & $\mathbf{4 . 3}$ & 2 \\
3 & 4.8 & 3 & 4.7 & 3 \\
4 & 5.2 & 4 & 5.5 & 4 \\
5 & $\mathbf{6 . 2}$ & 5 & $\mathbf{7 . 1}$ & 5 \\
\hline
\end{tabular}

${ }^{*}$ Mode description: 1. Left and right swinging of dish

2. Bending of dish

3. Up and down nodding of the dish

4. Rotating around the optical axis of the dish

5. Bending of dish

analysis. Figure 4.10 displays the natural frequencies of the first five modes for the ANSYS and GENESIS models as well as two cases, where $18 \%$ and $40 \%$ of the GENESIS model's rigid elements were removed. Note that the first and second modes of all three GENESIS models have switched with the ANSYS model's.

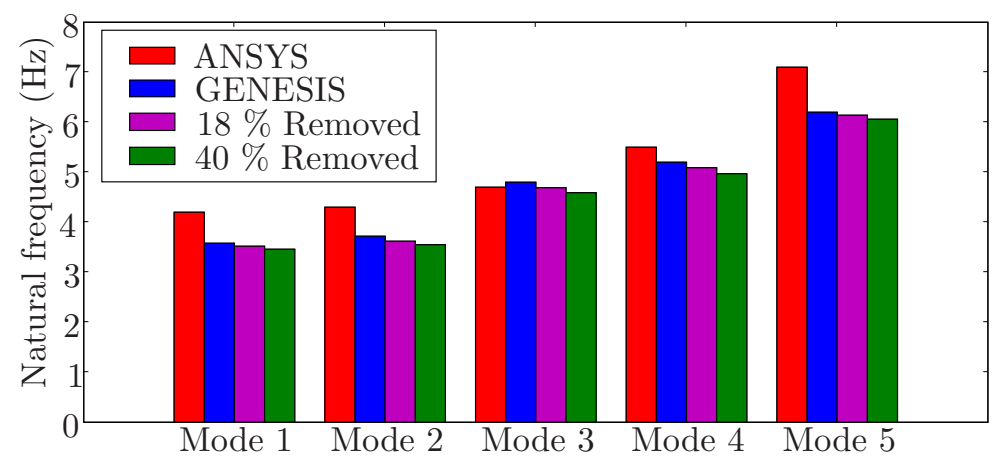

Figure 4.10: A comparison between the natural frequencies of the ANSYS- and GENESIS models and two cases of rigid element removal

When rigid elements are removed from the GENESIS model, there is a decline in the natural frequency values. By removing rigid elements from the model, one actually lowers the stiffness of the GENESIS model. It was concluded that the differences in the natural frequencies between the two models, can be explained in part due to the connection of the dish to the backing structure with an insufficient number of rigid elements. 
The two finite element models are not identical as certain changes had to be made to the GENESIS model as discussed in Section 4.3. Therefore the two models will not generate identical results. The GENESIS model was only changed where it was absolutely necessary. It resulted in producing similar results to the ANSYS model as was shown in this section.

The differences in results described here are acceptable as the focus of this study is not to duplicate the ANSYS model precisely or give a final design for the KAT antenna. One of the goals is to illustrate the different applications of structural optimisation where the GENESIS finite element model will be used as the baseline design. All new designs will be compared to this baseline, to identify any improvement on the design. This will not be influenced in a difference between the GENESIS- and ANSYS models. Secondly, the aim is to reduce the mass of the KAT antenna. Any mass that is saved from the GENESIS model will give a good indication of the mass that can be saved on the ANSYS model as the analyses gave similar results. 


\section{Chapter 5}

\section{External program}

GENESIS has the capability to call an user defined function to calculate responses that are not automatically calculated by GENESIS itself. This functionality allows the structural optimisation code to consider results for non-structural disciplines during the optimisation process. An external program was developed by the author to calculate the non-structural responses for each design cycle of the optimisation process. The code was written in Python.

\subsection{Overview of the external program}

The external program can be divided into two sections (as shown in Figure 5.1). The first section is referred to as the "analysis section". After the finite element analysis is completed, the non-structural responses are evaluated by calling this analysis section. The second section is termed, the "gradient section". This section is called during the sensitivity analysis, when the gradients of all constraints, including the non-structural constraints, are calculated. The analysis and gradient sections of the external program will be discussed in more detail in the following paragraphs.

\subsubsection{The analysis section}

GENESIS generates an output file called the punch file that contains the displacements and rotations of the finite element model after the finite element analysis has been completed. After the punch file is created, the analysis section of the external program is called for evaluation of the non-structural responses. During the analysis section (see Figure 5.2), the dish displacements and rotations are read by the external program from the punch file and a least squares fit of a paraboloid is made through the displaced grids. This idealised paraboloid of 


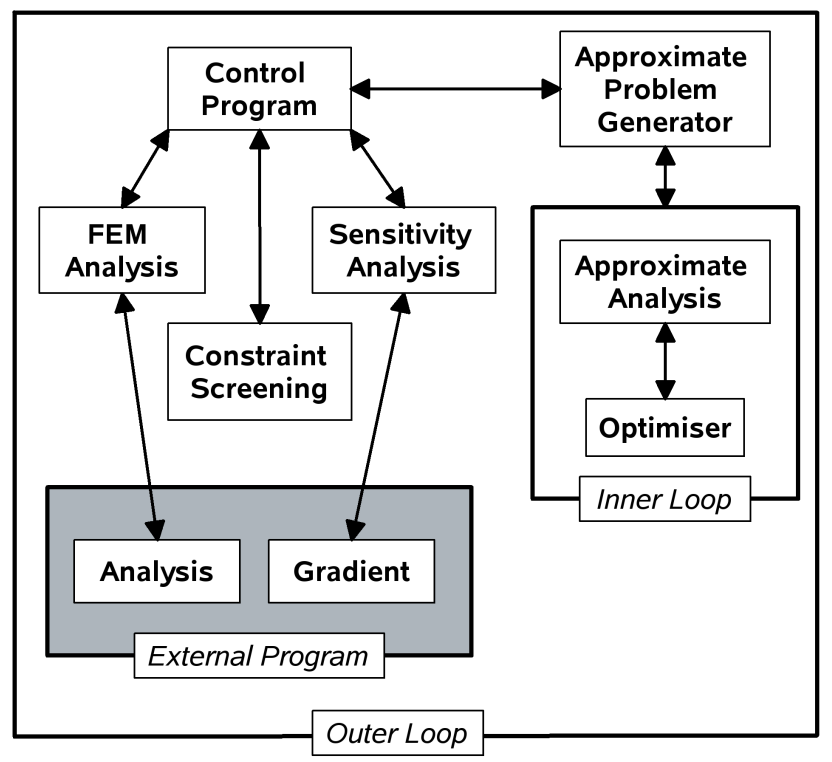

Figure 5.1: Coupling of GENESIS with the external program

the actual grid displacements, are used to calculate the non-structural responses. This process is repeated for each loadcase and the non-structural responses are sent back to GENESIS.

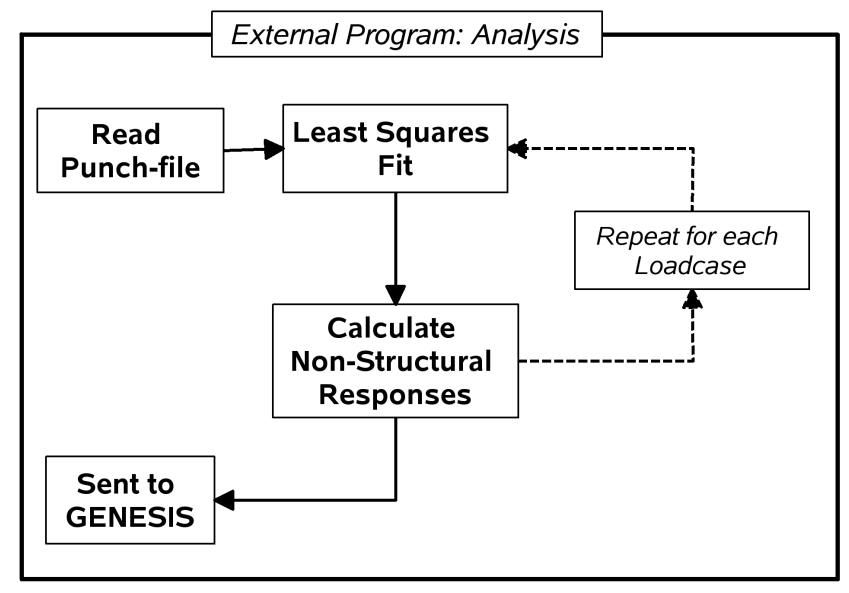

Figure 5.2: The analysis section of the external program

\subsubsection{The gradient section}

GENESIS implements analytic methods to calculate gradients for structural responses. However, GENESIS determines the gradients for the non-structural responses with finite difference gradients, since the analytic relationship of the 
non-structural responses is not available to it. This can be computationally expensive if the analyses required for finite differences are executed serially.

The coupling of the external program with GENESIS produced non-structural responses. During optimisation, gradients needs to be calculated for these responses. At first, GENESIS was used for the non-structural gradient calculations, but it was found to be computationally expensive. The reason for this is that a full finite element analysis must be performed for each gradient function evaluation and GENESIS executed these finite element analyses in series. It was decided to implement these finite difference calculations externally to GENESIS to enable the use of parallel computations.

The purpose of the gradient section is to calculate gradients with finite differences and parallelise the required finite difference analyses. In order to understand the operation of the gradient section, finite difference calculations will be discussed first. Thereafter the rest of the gradient section will be presented.

It was decided to implement finite difference gradients for the gradient calculations, since it can be easily parallelised as the function evaluations are independent of each other. The disadvantage is that it will dominate the computational time during optimisation, because of the many finite element analyses that are required [33]. A growth in the number of design variables will also lead to a large increase in computational time. However, these disadvantages will diminish if the finite difference calculations can be parallelised.

It was decided to use forward difference calculations, instead of central difference calculations. The total number of analyses required for forward difference calculations are equal to the number of independent design variables. For central difference calculations, the number of analyses required are equal to twice the number of design variables. Therefore forward difference calculations will lead to a shorter computational time as compared to central difference calculations. Note that each finite difference analysis is a finite element analysis which is time consuming.

The calculation of forward finite difference gradients can be illustrated, by considering a function $F$, that depends on a single variable $X$. The gradient of $F$ at the point $X_{0}$ is determined by (5.1.1) [33]:

$$
\left.\frac{d F(X)}{d X}\right|_{X=X_{0}}=\lim _{X \rightarrow X_{0}} \frac{F(X)-F\left(X_{0}\right)}{X-X_{0}}
$$

An approximation for the gradient of $F$ at the point $X_{0}$, can be made by perturbing $X$ with a small value, $h$, as shown in (5.1.2) [33]: 


$$
\left.\frac{d F(X)}{d X}\right|_{X=X_{0}} \approx \frac{F\left(X_{0}+h\right)-F\left(X_{0}\right)}{h}
$$

When $F$ is a function of more than one design variable, (5.1.2) is repeated for each design variable. For instance, if an optimisation problem consists of $n$ design variables then $n$ function evaluations are required to perform the finite difference calculations.

The gradient section consits of the following steps as displayed in Figure 5.3. Firstly, the step-size $(h)$ by which each design variable is perturbed, is specified. Next, each design variable is perturbed with the specified step-size. Thereafter, the perturbed design variables are used to update new finite element analysis data files and the corresponding finite element analyses are performed. In the first data file, only the first design variable is perturbed, while the other design variables remain unchanged. Similarly, only the second design variable is perturbed in the second data file. This is repeated for all data files.

A Python module, called DRMAA (Distributed Resource Management Application API), is called to simultaneously execute all of the finite element analyses in parallel. The Stellenbosch University cluster is used for the parallelisation. The number of finite element jobs that are submitted to the cluster is equal to the number of design variables $(n)$.

After the finite element analyses are completed, the analysis part of the external program is called for each finite element analysis to determine the corresponding non-structural responses. Finally, the finite difference calculations are performed. With the finite difference calculations finished, the gradient information for each non-structural response are sent back to GENESIS. Thereafter the approximate problem generation will follow as shown in Figure 5.1.

In the following sections, more detail will be provided for the specific parts of the external program. This includes an explanation of the least squares fit, the actual calculation of the non-structural responses, the step-size selection and the methods used to implement the parallel computing.

\subsection{Least squares fit}

The non-structural responses determine if the deformed dish actually focuses on the deformed FPA. If this is not the case, then the FPA will not be able to detect the signal reflected from the dish. The focus point of the undeformed dish is calculated from (3.3.1). (3.3.1) can not be directly applied to the deformed dish, since it changes shape when loaded. Therefore, a least squares fit of the 


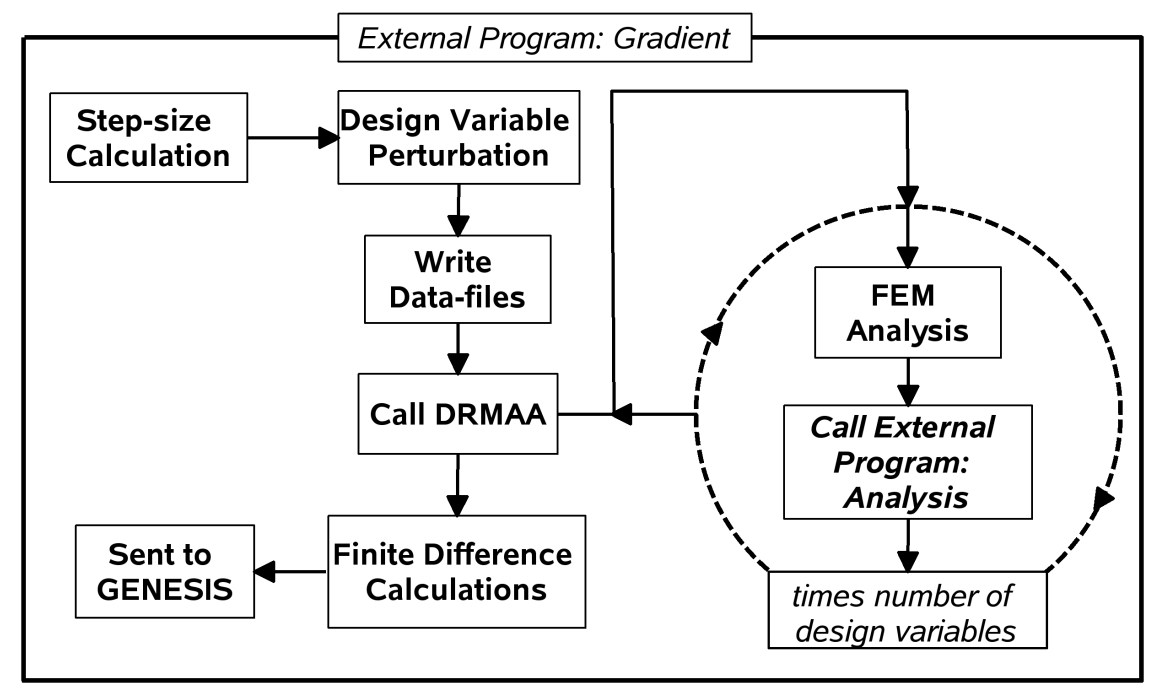

Figure 5.3: The gradient section of the external program

form shown in (3.3.1), was performed on the deformed dish. It fits an idealised paraboloid through all the deformed dish grid points. Thus, the focus point of the deformed dish is approximated by the focus point of the fitted paraboloid. Note that the smaller the error in the fit, the more accurate the prediction of the deformed dish's focus point will be.

Translational and rotational compensation were applied to remove bias and tilt from the fitted data. This can be accomplished by adding a series of Fourier polynomials, called Zernike polynomials [34] (which are commonly used in optics), to (3.3.1). The Zernike polynomials has the form shown in (5.2.1) [34].

$$
Z(x, y)=\sum_{j=1}^{M} C_{j} \phi_{j}
$$

A Zernike polynomial with only the lower order coefficients is shown in (5.2.2) [34]. The bias is given by the constant $d$ and the $Y$ - and $Z$-tilt by the constants $b$ and $c$ respectively.

$$
Z(y, z)=b y+c z+d
$$

Different combinations of the Zernike polynomials were added to (3.3.1), to apply translational correction along the $X$-axis and rotational compensation along the $Y$ - and $Z$-axes. To illustrate the process that was followed, consider the KAT antenna at zero pitch and azimuth with only a gravity load. Figures 5.4(a) - 5.4(c) show the results of the least squares fits, with no compensation, translational compensation along the $X$-axis and combined translational- and rotational along the 
$X$-axis and the $Y$ - and $Z$-axes, respectively. These figures display a zoomed plot (at the middle point of the dish) of the grids on the vertical parabola of the dish in the $X Z$-plane.

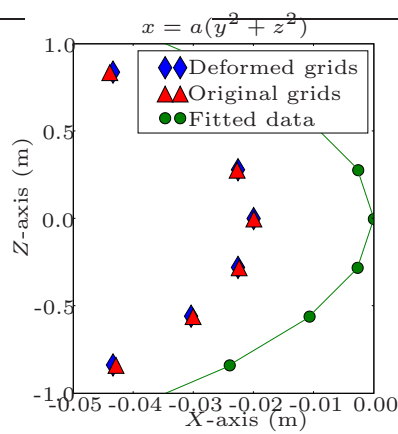

(a) No compensation

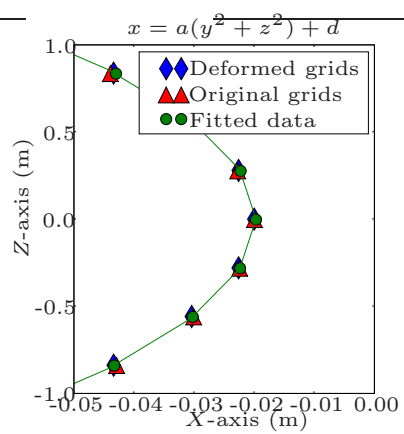

(b) Translational compensation along the $X$-axis

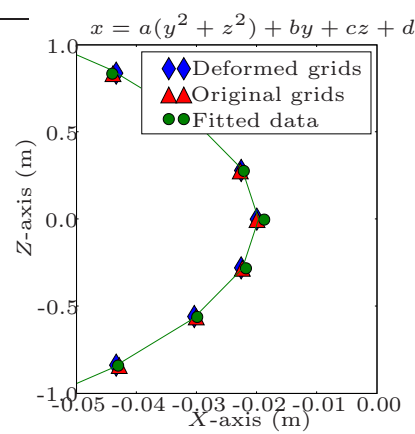

(c) Translational- $(X)$ and rotational ( $Y$ and $Z$ ) compensation

Figure 5.4: Three least squares fits with different levels of compensation

Table 5.1: A comparison of the results from the least squares fits

\begin{tabular}{ccccc}
\hline Parameter & Undeformed $^{a}$ & Fit- $^{b}$ & Fit-2 $^{c}$ & Fit-3 $^{d}$ \\
\hline \hline$a$ & -0.03333 & -0.03393 & -0.03333 & -0.03333 \\
$b$ & 0.0 & - & - & $2.4 \mathrm{E}^{-6}$ \\
$c$ & 0.0 & - & - & -0.0012 \\
$d$ & 0.0 & - & -0.02103 & -0.02104 \\
RMS-of-xyz-error (m) & 0.0 & 0.0119 & 0.0044 & 0.0004 \\
RMS-error-to-focus (m) & 0.0 & 0.0052 & 0.0016 & 0.0002 \\
\hline
\end{tabular}

${ }^{a}$ Parameters for the undeformed dish

${ }^{b}$ Least squares fit with no compensation

${ }^{c}$ Least squares fit with translational compensation along the $X$-axis

${ }^{d}$ Least squares fit with translational- $(X)$ and rotational $(Y, Z)$ compensation

Table 5.1 displays the constants and errors attained for each least squares fit as well as for the undeformed dish. The RMS-of-xyz-error shows how accurate the least squares fit represents the original paraboloid. The RMS-error-to-focus is the dish surface error as explained in Section 3.3. Therefore a decrease in these two values will result in a more accurate least squares fit.

From figure 5.4(a), it is clear that there is a constant offset between the fitted curve and the deformed and undeformed dish. The reason for this is that the centre of the front face of the dish is not located on the zero point of the axis system. There is a difference of $20 \mathrm{~mm}$ which explains the offset in Figure 5.4(a). 
This offset is removed, by adding translational compensation in the $X$-direction, which results in Figure 5.4(b) with a zero offset. For Fit-2 in Table 5.1, $d$ is equal to $-21 \mathrm{~mm}$, indicating the translational compensation in the $X$-direction was successful. The fit is improved by allowing translational compensation along the $X$-axis and rotational compensation along the $Y$-and $Z$-axes. This results in a small compensation along the $Z$-axis and almost no compensation along the $Y$-axis ( $c$ and $b$ of Fit-3 in Table 5.1). As the improvements are quite small, the change is not visible in Figure 5.4(c). The RMS-of-xyz-error and the RMS-errorto-focus decreased with each fit, indicating that the next fit was better than the previous one.

One could continue by adding higher order Zernike coefficients which are known as aberrations. The aberrations only compensate for image degradation and will not improve the structural characteristics of the fit. Therefore no more compensations were implemented for the least squares fit, since adding more Zernike polynomial terms would not improve it.

\subsection{Non-structural responses calculation}

Calculation of the non-structural responses can be divided into two parts. The first part is the calculation of the dish surface error (the RMS and maximum as presented in Section 3.3) and the second part is the calculation of the relative FPA displacements and rotations (refer to Section 3.3). Calculation of the dish surface error will be explained first where after the relative FPA displacements and rotations will be covered. Note that all formulae used for the non-structural response calculations were obtained from [29].

An illustration of the dish surface error calculation, is shown in Figure 5.5. The blue parabola is the undeformed dish and the red parabola is a quadratic curve fit through the grids of the deformed dish. The yellow dot is the same grid (i) on the two different parabolas. The blue and red dots are the focus points of the blue and red parabolas respectively. Consider (5.3.1), which calculates the difference between the focal distances for each grid point $(i)$ on the dish's front face, of the deformed case and the fitted case. $u f_{d}^{i}$ and $f f_{d}^{i}$ are shown in Figure 5.5.

$$
\triangle f_{d}^{i}=u f_{d}^{i}-f f_{d}^{i}
$$

To determine the dish surface error, (5.3.1) is calculated for each grid point first. Thereafter the RMS is calculated to determine the RMS dish surface error 


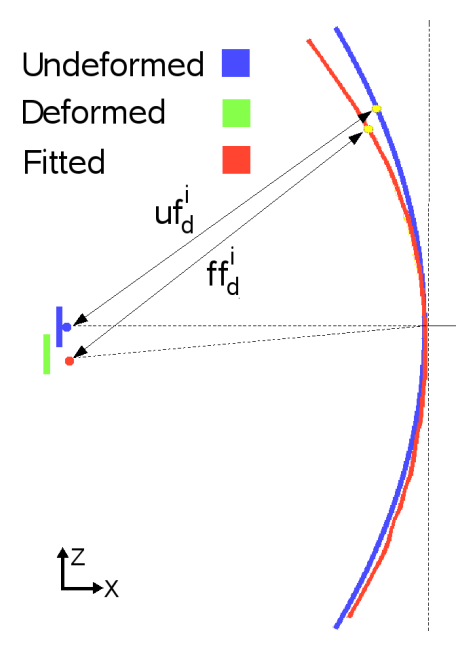

Figure 5.5: An Illustration of the dish surface error calculation

as shown in (5.3.2) where $g$ is the total number of grid points on the dish's front face.

$$
D S E_{r m s}=\sqrt{\frac{\left(\triangle f_{d}^{i}\right)^{2}+\left(\triangle f_{d}^{i+1}\right)^{2}+\ldots+\left(\triangle f_{d}^{g}\right)^{2}}{g}}
$$

To determine the maximum surface error, the maximum is calculated instead of the RMS as given by (5.3.3).

$$
D S E_{\max }=\max \left(\triangle f_{d}^{i}, \triangle f_{d}^{i+1}, \ldots, \triangle f_{d}^{g}\right)
$$

The procedure that was followed to calculate the FPA displacements and rotations is illustrated in Figure 5.6 (zoomed in from Figure 5.5). In Figures 5.6(a) and 5.6(b), the blue rectangle (or disk) represents the undeformed FPA and the green rectangle (or disk) the deformed FPA. The blue and red dots are the focus points of the undeformed dish and the fit to the deformed dish respectively. The finite element analysis provides the FPA translation and rotation. The $X$ displacement and $Y$ - and $Z$-rotations of the focus point are obtained from the least squares fit. The difference between the two focus points are subtracted from the difference between the two FPA's for the $X$-direction. This provides the relative FPA displacement in the $X$-direction as shown by (5.3.4). The same is done for the $Y$ - and $Z$ relative FPA rotations as shown by (5.3.5) and (5.3.6) respectively.

$$
\begin{gathered}
T X_{r e l}=T X_{F P A}-T X_{f p} \\
R Y_{r e l}=R Y_{F P A}-R Y_{f p}
\end{gathered}
$$




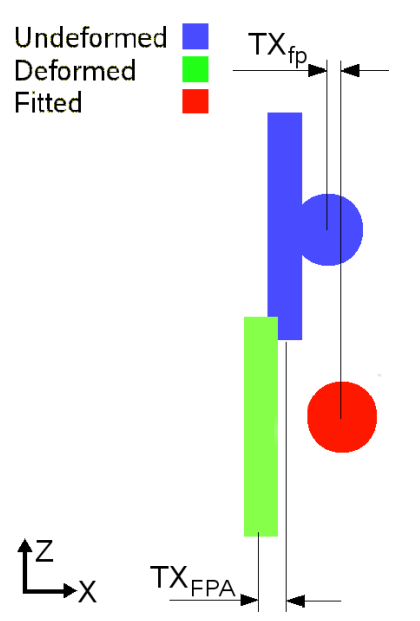

(a) Side view

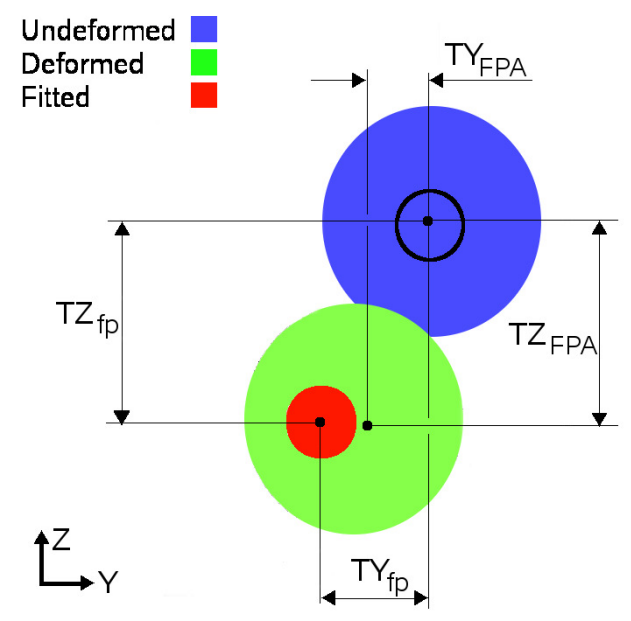

(b) Front view

Figure 5.6: An Illustration of the relative FPA displacement calculation

$$
R Z_{r e l}=R Z_{F P A}-R Z_{f p}
$$

The $Y$ - and $Z$-displacements of the focus point are obtained by applying simple trigonometry to the $Y$ - and $Z$-rotations as shown in (5.3.7) and (5.3.8). An illustration of these displacements is shown by Figure 5.6(b).

$$
\begin{aligned}
& T Y_{f p}=\tan \left(R Z_{f p}\right) f_{d} \\
& T Z_{f p}=\tan \left(R Y_{f p}\right) f_{d}
\end{aligned}
$$

The relative FPA $Y$ - and $Z$-displacements are calculated (shown by (5.3.9) and (5.3.10)) similarly as the relative FPA $X$-displacements.

$$
\begin{gathered}
T Y_{\text {rel }}=T Y_{F P A}-T Y_{f p} \\
T Z_{r e l}=T Z_{F P A}-T Z_{f p}
\end{gathered}
$$

Finally, the displacement in the $Y Z$-plane is calculated by applying (5.3.11).

$$
Y Z_{r e l}=\sqrt{T Y_{r e l}^{2}+T Z_{r e l}^{2}}
$$

The only non-structural response that was not calculated is the relative FPA $X$-rotation. No information was provided regarding the calculation of this response for the ANSYS finite element model. The Zernike polynomials are also not capable in producing this response. The average rotation around the optical axis of all the dish grids was calculated as $0.00029^{\circ}$. The largest FPA rotation around the optical axis is $0.0007^{\circ}$. This shows that the FPA and dish experience very small rotations about the optical axis. Therefore it was decided to 
exclude the relative FPA $X$-rotation from the optimisation, since the optical axis rotations are insignificantly small.

As the GENESIS- and ANSYS finite element models did not give the same results, one expects that the non-structural responses would differ. A comparison between the non-structural responses for the ANSYS- and GENESIS models are shown in Table 5.2, for loadcase A (the values smaller than $1.0 \mathrm{E}^{-3}$ are noted as zero).

The FPA movement relative to the fitted parabola and focus point differ by a noticeable amount between the GENESIS- and ANSYS finite element models. This can be ascribed to the differences between the FPA movement and fitted parabola focus point movements for both models (printed in bold). Firstly these differences exist due to the uncertain procedure that was followed to determine the focus point movements from the least squares fit for the ANSYS model. Secondly, a rigid element was inserted into the GENESIS model to ensure that the FPA is at the focus point of the unloaded structure (see Section 4.3.3), which was not the case with the ANSYS model. Thirdly, the temperature loads between the two models produce different results (see Section 4.4), which will result in different non-structural responses.

Table 5.2: A comparison of the relative FPA displacements and rotations [29]

\begin{tabular}{|l|ccc|ccc|ccc|}
\hline & \multicolumn{3}{|c|}{$\begin{array}{c}\text { Fitted parabola } \\
\text { focus point } \\
\text { movements }\end{array}$} & \multicolumn{2}{|c|}{ FPA movement } & \multicolumn{3}{c|}{$\begin{array}{c}\text { FPA movement } \\
\text { relative to } \\
\text { fitted parabola } \\
\text { focus point }\end{array}$} \\
\hline \hline Transla- & UX & UY & UZ & UX & UY & UZ & UX & UY & UZ \\
tions & $\mathrm{mm}$ & $\mathrm{mm}$ & $\mathrm{mm}$ & $\mathrm{mm}$ & $\mathrm{mm}$ & $\mathrm{mm}$ & $\mathrm{mm}$ & $\mathrm{mm}$ & $\mathrm{mm}$ \\
\hline ANSYS & $\mathbf{9 . 5}$ & $\mathbf{- 1 3 . 9}$ & -0.05 & 1.52 & 0.0 & $\mathbf{- 1 . 9}$ & -7.98 & 13.9 & -1.95 \\
GENESIS & $\mathbf{1 4 . 2}$ & $\mathbf{- 8 . 8}$ & -0.03 & 0.04 & -0.02 & $\mathbf{- 1 3 . 3}$ & -14.16 & 8.78 & -13.27 \\
\hline \hline Rota- & $\mathrm{RX}$ & $\mathrm{RY}$ & $\mathrm{RZ}$ & $\mathrm{RX}$ & $\mathrm{RY}$ & $\mathrm{RZ}$ & $\mathrm{RX}$ & $\mathrm{RY}$ & $\mathrm{RZ}$ \\
tions & $\mathrm{deg}$ & $\mathrm{deg}$ & $\mathrm{deg}$ & $\mathrm{deg}$ & $\mathrm{deg}$ & $\mathrm{deg}$ & $\mathrm{deg}$ & $\mathrm{deg}$ & $\mathrm{deg}$ \\
\hline ANSYS & - & 0.0 & $\mathbf{- 0 . 1 0 6}$ & 0.0 & $\mathbf{- 0 . 0 0 5}$ & 0.0 & - & -0.005 & 0.106 \\
GENESIS & - & 0.0 & $\mathbf{- 0 . 0 6 7}$ & 0.0 & $\mathbf{0 . 0 2 2}$ & 0.0 & - & 0.022 & 0.067 \\
\hline \hline
\end{tabular}

\subsection{Step-size calculation}

To calculate the sensitivity of the non-structural responses with finite differences, a finite difference step-size is required. The step-size calculation is very important 
to ensure an accurate approximation of the gradient.

According to Haftka [35], two sources of errors exist when gradients are approximated with finite difference calculations. The first error, is termed the truncation error, which is a result of the neglected terms in the Taylor series expansion during the calculation of the perturbed function. The second kind of error is termed the condition error, which is the difference between the numerical evaluation of the function and its exact value. These two errors lead to the so called "step-size dilemma". If one wants to reduce the truncation error, by decreasing the step-size, it will result in an increase of the condition error.

Haftka presents a formula to calculate the optimum step-size, but it was decided to follow the same method that GENESIS implements. GENESIS uses two constants in the step-size calculation, namely the relative step-size and the minimum step-size (the default values are 0.00001 and 0.000001, respectively). Each design variable is multiplied by the relative step-size. If the product is larger than the minimum step-size, then the step-size for that specific design variable is equal to the calculated product. When the product is smaller than the minimum step-size, the step-size is set equal to the minimum step-size.

The minimum step-size will ensure that the selected step-size is not too small, which limits the condition error. On the other hand, the relative step-size will prevent a large truncation error as it scales the step-size relative to the design variable value.

The selection of the relative- and minimum step-size constants will be dependent on the size of the design variable values. The design variables during the optimisation of the KAT ranged between $0.001(1 \mathrm{~mm})$ and $0.1(100 \mathrm{~mm})$. It was suspected that the default parameters used by GENESIS were too small for this application. Different values were tested for the step-size parameters and it was found that 0.001 and 0.0001 worked the best for the KAT optimisation. It resulted in a better optimum compared to the default step-size parameters used by GENESIS.

\subsection{Parallel processing}

Optimisation where GENESIS couples with an external program is equivalent to general purpose optimisation, since GENESIS can not determine the gradients of the external program's responses. Three categories were identified which take-up the computational time of general purpose gradients-based optimisers [33]:

- Analyses for one-dimensional search 
- Analyses for gradient calculation

- Other optimisation computations

The total solution time is dominated by the analyses for gradient calculations and one-dimensional searches, if a moderate amount of design variables are used. The one-dimensional search is fairly independent of the number of design variables. This is not the case with the finite difference calculations as the number of analyses increase with an increase in the number of design variables. Finite difference calculations will dominate the optimisation time if more than a couple of design variables are used [33].

This is exactly what was seen during the optimisation of the KAT. The largest part of the optimisation time was dominated by the calculation of the analyses for the finite difference gradients. A two level parallelisation approach was implemented to lower the computational time. For the first level, shared memory processing was applied, during which more than one processor/core on a single compute node was used for each analysis. The second level implemented distributed memory processing, whereby the analyses for gradient computations were ran in parallel on multiple compute nodes. Before a discussion of these two methods, consider first the cluster that was used for the parallel computing.

The parallel processing were executed on the Stellenbosch University cluster. It consists of 1 head node and 21 compute nodes. Each compute node consists of 8 cores resulting in a cluster with 168 cores. Thus shared memory processing can be executed with a maximum of eight cores and distributed memory processing with a maximum of 168 cores. Jobs are submitted on the head node which distribute tasks to the compute nodes. Sun Grid Engine 6.0 is used as the job scheduler.

GENESIS has a build-in capability to automatically divide an analysis between more than one processor in a shared memory processing environment. This level of parallelisation can be turned on or off using a command line switch. Instead of using only one processor for an analysis, up to eight processors can be utilised using the Stellenbosch cluster. This is very helpful as most of the computational time is consumed by executing an analysis, either for the one-dimensional search or for finite difference calculations.

The optimal number of processors was investigated by optimising a KAT component using a different number of processors each time. Consider optimisation of the KAT yoke with 11 design variables (more details about the yoke optimisation can be seen in Section 6.2.1). Figure 5.7 shows the computational time for optimisation of the yoke with one-, two-, four-, six-, and eight processors. It is interesting to see that the computational time decreased from one- to six 
processors, but there is an increase between six- and eight processors. Although six processors were faster than four processors, it was decided to only use four processors for the analyses. The reason being that the decrease in computational time is very small when moving from four to six processors. The use of four processors will also match the hardware configuration better. Each compute node has eight cores which enables the execution of two, four processor finite element analyes on a single compute node. This becomes especially important for the second level parallelisation.

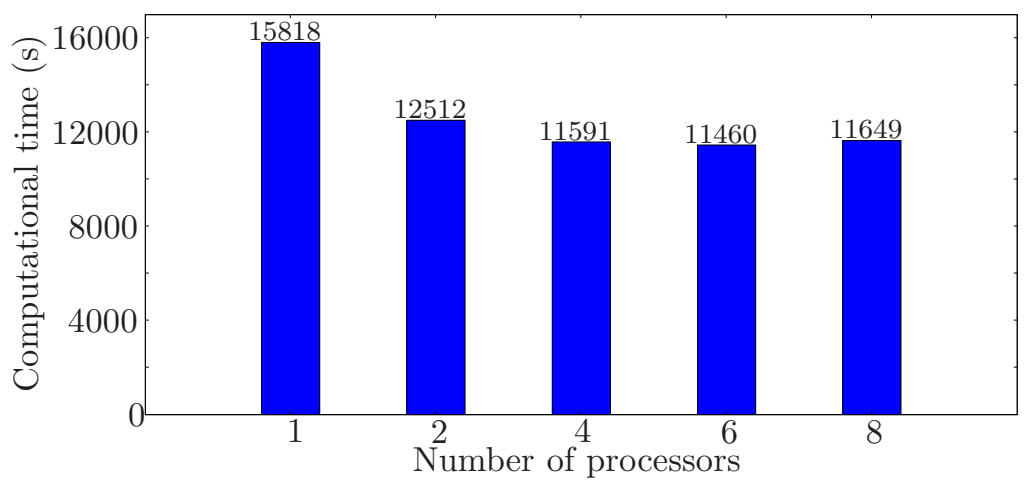

Figure 5.7: Yoke optimisation with a different number of processors

A second level of parallelisation was implemented to speed-up the finite difference calculations even more. The finite difference calculations are all independent and can be executed simultaneously. Note that the first level of parallelisation was still utilised with the first level.

A master-worker paradigm was used for the second level of parallelisation. The master processor, which executes the optimisation, allocates tasks to the worker processors whenever necessary for multiple analyses. When the worker is finished with the analysis, another task is allocated until all tasks are completed. This structure was chosen as it requires minimal inter-processor communication. Only the design variables are sent to each worker which in turn sends the responses back to the master.

For the second level of parallelisation, the DRMAA module is called to submit multiple finite element analyses on the cluster. The DRMAA module is an Application Protocol Interface (API) for the submission and control of jobs to Distributed Resource Management (DRM) systems. DRMAA waits until all the jobs are finished and only then will the external program continue with the rest of the code. 
To illustrate the decrease in computational time that is produced by combining the first and second levels of parallelisation, consider again the example of the yoke with 11 design variables. Figure 5.8 shows a plot of the computational time against four cases of processing: serial processing (1 processor), four processors used for the analyses, parallel processing of the finite difference analyses (12 processors) and the combination of four processors for the analyses and parallelised finite difference analyses (48 processors). It can clearly be seen from Figure 5.8 that both levels of parallelisation leads to a large reduction in computational time. For the case where both levels were implemented, 48 processors were used which leads to a computational time reduction of $58 \%$ compared to the serial processing. Note, that with an increase in design variables, the parallelisation will reduce the computational time even more.

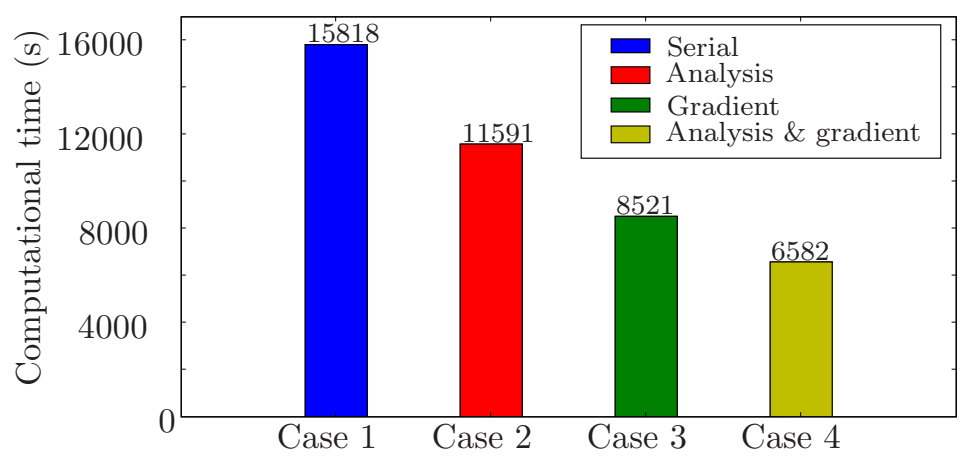

Figure 5.8: Yoke optimisation with different levels of parallelisation

The implementation of the parallel processing did not produce exactly the same results as the serial processing. The parallel- and serial optimisation did generally not converge after the same number of design cycles. The final design points for the two processes were also not identical. In theory, both processes should give the same answer.

Consider two of the cases that were investigated to show the difference in the results from the serial- and parallel processing. Note that the finite difference gradients for the serial processing was calculated internally by GENESIS, while the finite difference gradients for the parallel processing was calculated externally by the external program. The first case consists of optimising the yoke (see Section 6.2.1 for a description of the optimisation problem). For the second case, multiple components were optimised simultaneously, including the yoke, backing structure and FPA arms (see Section 6.2.4 for a description of the optimisation 
problem). Plots of the objective function (left) and maximum constraint violation (right) versus the design cycles are shown in Figure 5.9 for serial- and parallel processing. A red dot and blue dot indicate an infeasible design point for the parallel- and serial processing respectively. Whenever a design point is feasible, it is indicated with a green dot. For each infeasible design point, a bar chart is plotted showing the maximum constraint violation. A red bar and blue bar indicates the constraint violation for the parallel- and serial processing respectively. For both processes, the minimum objective function in the design process is shown with a green diamond as well as the specific value.

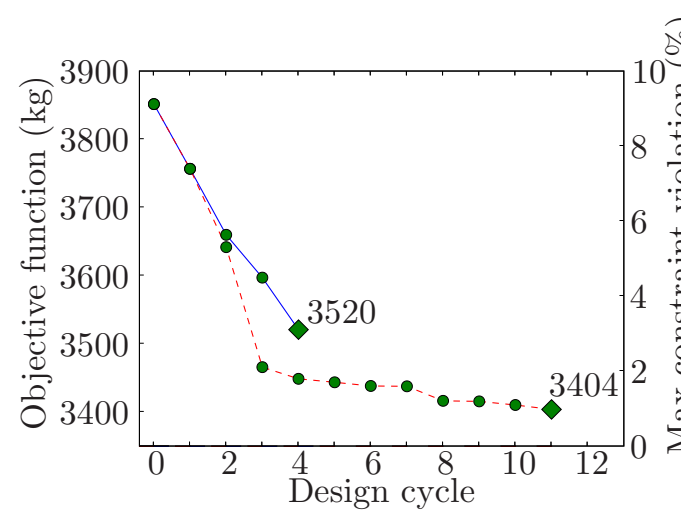

(a) Optimisation of the yoke

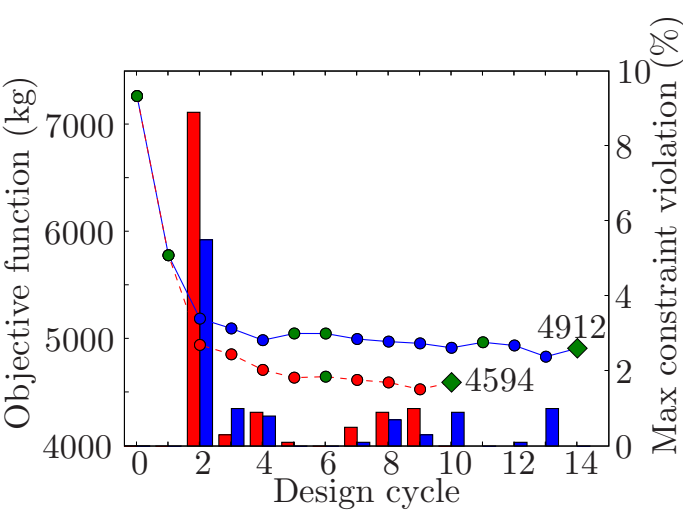

(b) Multiple component optimisation

- Optimum design point

- Feasible point

- Infeasible point: Serial processing

-. Infeasible point: Parallel processing

- Max. constraint violation: Parallel processing

- Max. constraint violation: Serial processing

Figure 5.9: Plots of the objective function and maximum constraint violation versus design cycles for the serial- and parallel processing

In Figure 5.9 it is clear that the same design point is found for both serialand parallel processing, for the first two design cycles. Thereafter the design points diverge to a small degree. The serial processing converges much earlier than the parallel processing for the yoke (see Figure 5.9(a)), but for the multiple component optimisation the opposite happens (see Figure 5.9(b)). Convergence is always at a feasible point and a better design point is found for parallel processing than the serial processing in both cases.

The differences between the serial- and parallel processing were further investigated, by firstly restarting the serial processing of the multiple component optimisation at design point 14 . The objective function was lowered by a small degree (to $4906 \mathrm{~kg}$ ). In theory, the optimiser should have reduced the objective function to a point which is near the parallel processing optimum after restarting 
the optimisation, unless it is caught in a local minimum, which is probably the case here. Secondly, serial optimisation was executed by starting at a design point (design point six) of the parallel optimisation. The optimiser found an optimum of $4575 \mathrm{~kg}$, which is very near the optimum of the parallel optimisation.

It was identified that the difference between the two processes are most likely related to the calculation of the gradient information. The gradients are calculated externally for the parallel implementation while they are calculated internally by GENESIS for the serial computations. Even a small difference in the gradient calculations could lead to a different search direction which may result in a different path to the optimum (or even to a different optimum). A detailed study of the iteration results showed that the design points are initially the same. After two design cycles a noticeable difference occurs which ends in a small divergence.

The implementation of the parallel processing is still valid as it provides the same or a better design point compared to the serial processing. Although the parallel processing converges after a larger number of design cycles for some cases, the computational time is still much less compared to the serial processing. Figure 5.10 shows the difference in computational times for the serial- and parallel processing for each of the investigated cases including the backing structure. For the multiple component optimisation the parallel processing is 14 times faster than the serial processing. This is an indication of how powerful the parallel processing is.

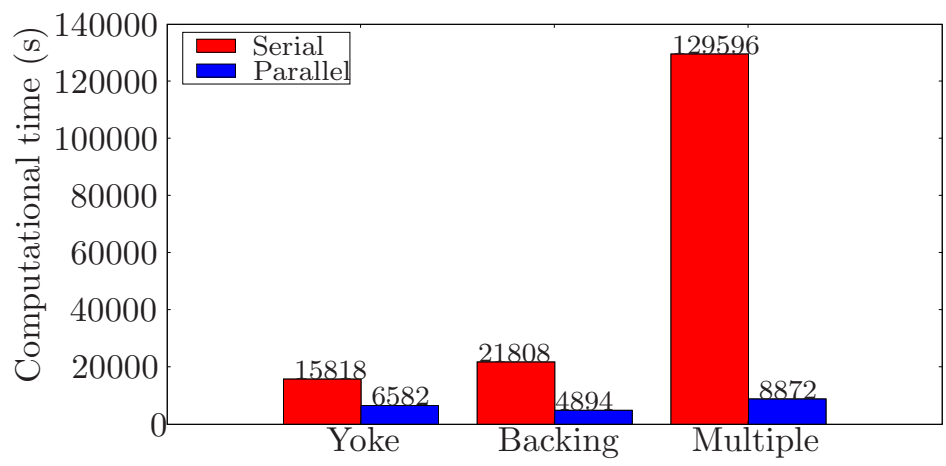

Figure 5.10: A comparison between serial- and parallel processing for various cases of optimisation 


\section{Chapter 6}

\section{KAT optimisation}

This chapter starts with a discussion of the objective function and constraints that were implemented during the optimisation of the KAT. Thereafter the optimisation results are presented. The optimisation results are divided into four categories, according to the different structural optimisation applications.

Mass minimisation was implemented on the yoke, backing structure and FPA arms of the KAT antenna. A new concept was generated for the backing structure, utilising topology- and size optimisation. Four concepts, for the yoke and backing structure each, were optimised and compared. Design for structural feasibility is illustrated by means of the KAT antenna.

The design problems presented in this study are variations of the existing KAT prototype (shown in Figure 3.1). The GENESIS finite element model, as discussed in Section 4.3, will be used for the finite element analyses during the optimisation. The optimised KAT antenna will be compared relative to the initial GENESIS finite element model. Note that the initial thickness values were identical to that of the prototype, for all the size optimisations implemented in this chapter.

\subsection{Optimisation problem definition}

Based on the requirements of Section 3.3, the optimisation problem specifications are provided in terms of the objective function and constraints that were used.

\subsubsection{Objective function}

The objective function considered in this work, for all size- and shape optimisation problems, was mass minimisation. This is consistent with the overall goal of reducing material costs as much as possible, while satisfying all the design 
requirements. For topology optimisation, the objective function was to minimise the strain energy which will lead to a stiffer structure.

\subsubsection{Constraints}

The constraints for the size- and shape optimisation can be divided into two groups: structural constraints and non-structural constraints. GENESIS can not implement non-structural constraints during topology optimisation. Therefore only structural constraints were used during the topology optimisation of the KAT. The structural constraints are the responses that GENESIS is capable of calculating internally, including stresses and natural frequencies. The nonstructural constraints include the dish surface errors and relative FPA displacements and rotations, which are the responses that GENESIS can not calculate.

Since the prototype is known to satisfy all the stress design requirements, it was decided to modify the design, while restricting the stress to be no worse than that of the prototype. The natural frequency was constrained as shown in Section 3.3. Some of the non-structural responses obtained from the prototype finite element model were larger than the design requirements. During the optimisation, these responses were limited to be no worse than that obtained from the prototype finite element model. All other design specifications were implemented as specified (except the FPA relative $X$-rotation which was negligible small). The structural- and non-structural constraints will be discussed in more detail in the subsequent paragraphs.

\section{Structural constraints}

For the topology optimisation, mass fraction- and fabrication constraints were utilised. Stress- and a natural frequency constraint were implemented as the structural constraints for size- and shape optimisation. First the structural constraints for the topology optimisation will be discussed whereafter the structural constraints for the size- and shape optimisation will be presented.

The mass fraction constraint is the fraction of the structure's mass that should be kept during topology optimisation. For the fabrication constraint, symmetry was enforced about the $X Y$ - and $X Z$-planes.

For the size- and shape optimisation stress constraints, the highest Von Mises stress values (for normal operating conditions) were calculated for each component from the baseline finite element model. The GENESIS finite element model of the prototype, as discussed in Section 4, was used as the baseline. This stress value is then used as a design constraint for the specific component during the 
optimisation process. The value used for the upper bound will thus differ for each component as summarised in Table 6.1.

Table 6.1: Bounds on the stresses in each component

\begin{tabular}{cc}
\hline Component & Maximum Stress (MPA) \\
\hline \hline Yoke & 123.6 \\
Backing structure & 130.5 \\
FPA arms & 112.00 \\
\hline
\end{tabular}

A maximum Von Mises stress constraint, calculated at a wind speed of $160 \mathrm{~km} / \mathrm{h}$, was used to ensure that the altered design will survive the survival conditions as specified in Section 3.3. This stress (survival conditions) was calculated at a different load in comparison with the stress constraint (normal conditions) in the previous paragraph. For this loadcase, all steel components were constrained with a maximum Von Mises stress of $250 \mathrm{MPa}$, which will guarantee a safety factor of 1.2.

The first natural frequency of the model was constrained to have a lower bound of $3 \mathrm{~Hz}$, as specified in the design specifications. No constraints were imposed on the dish as it was not designed and analysis of the prototype showed relatively low stresses.

\section{Non-structural constraints}

Constraints were placed on the dish surface errors and relative FPA displacements and rotations. The analysis of the prototype showed that some of the responses were larger than the specified design requirements. For these responses, the values obtained from the GENESIS finite element model of the prototype were taken as the bound. The bounds on the responses that were smaller than the design requirements, were taken as the design specification values.

The specific bounds on each non-structural response are shown in Table 6.2 (in the Upper/Lower bound rows, the positive value is the upper bound and the negative value the lower bound). The dish surface errors and the relative FPA rotations are constrained at the specified design requirement values. All of the relative FPA displacements bounds (printed in bold in Table 6.2) were set equal to the values obtained from the prototype analysis, except for the relative FPA $X$-displacement of loadcase C. For this case, the response obtained from the prototype analysis, was smaller than the design requirement and as a result the original design requirement was used as the bound. 
Table 6.2: Bounds for the non-structural responses

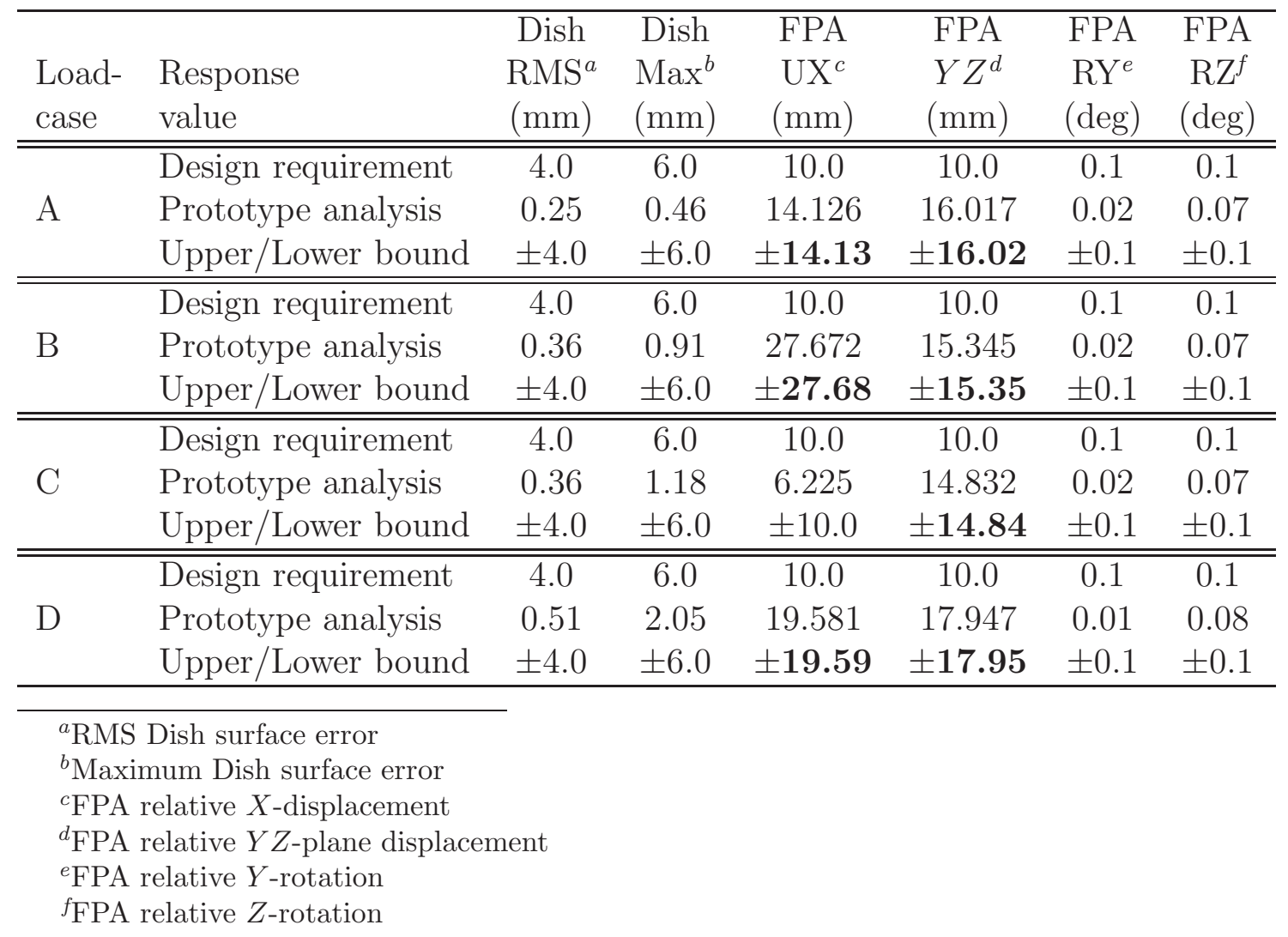

\subsection{Mass minimisation}

The goal of this section is to show a typical structural optimisation application where the mass of a structure is minimised. Size- and shape optimisation will be implemented which is considered as "fine tuning" the existing prototype design according to the design specifications. The result is a possible reduction in material costs, without an increase in manufacturing costs. First the yoke, FPA arms and the backing structure will be considered separately. Size optimisation will be applied to the yoke, while size- and shape optimisation will be done on the backing structure and FPA arms. Thereafter all three components will be optimised simultaneously.

\subsubsection{Yoke}

Size optimisation was done on the yoke without changing the original layout of the component. The goal was to reduce the material used, without increasing the maximum stress or non-structural responses of the component, as compared to the existing prototype and the original design specifications. There are 11 design 
variables which are the thicknesses of the different plates in the yoke. In Figure 6.1 the colours indicate the different design variables. Due to manufacturing constraints the thickness values were constrained to be larger than $1 \mathrm{~mm}$.

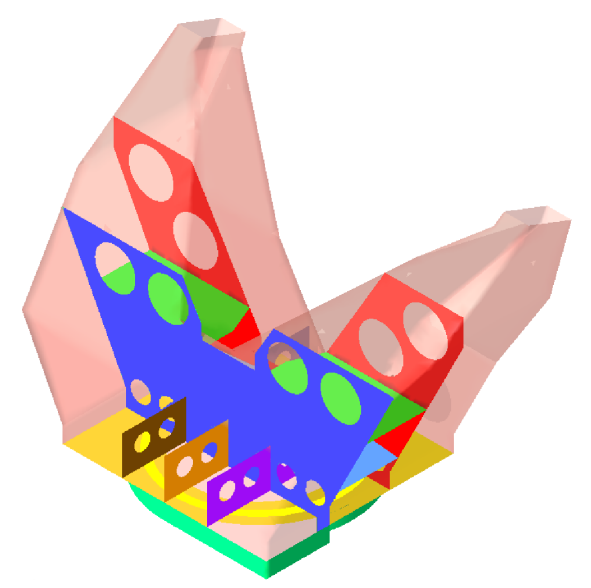

Figure 6.1: Original yoke with the colours indicating different properties

The results of the size optimisation are summarised in Figure 6.2 and Table 6.3. From Figure 6.2 it is clear that most design variables decreased, with a considerable decrease for design variable 2. Design variables 8 and 9 are almost at their lower bounds, indicating that they contribute very little to the overall strength or stiffness of the yoke. These design variables could be omitted from the structure, but it has to be determined if these plates contribute to the yoke's stiffness. The lower bounds of these design variables should be reduced (to $0.1 \mathrm{~mm}$ ) and optimisation should be repeated. If the design variables reaches the lower bound again, it indicates their corresponding plates can be removed from the yoke. There was a decrease of $12 \%$ or $447 \mathrm{~kg}$ in the mass of the yoke after 11 design cycles. All constraints are met for the final design, including the non-structural constraints.

Table 6.3: Mass results for the original yoke

\begin{tabular}{lc}
\hline Parameter & Value \\
\hline \hline Number of design cycles & 11 \\
Original mass (kg) & 3851 \\
Optimised mass (kg) & 3404 \\
Mass saved (kg) & 447 \\
Mass saved (\%) & $12 \%$ \\
\hline
\end{tabular}




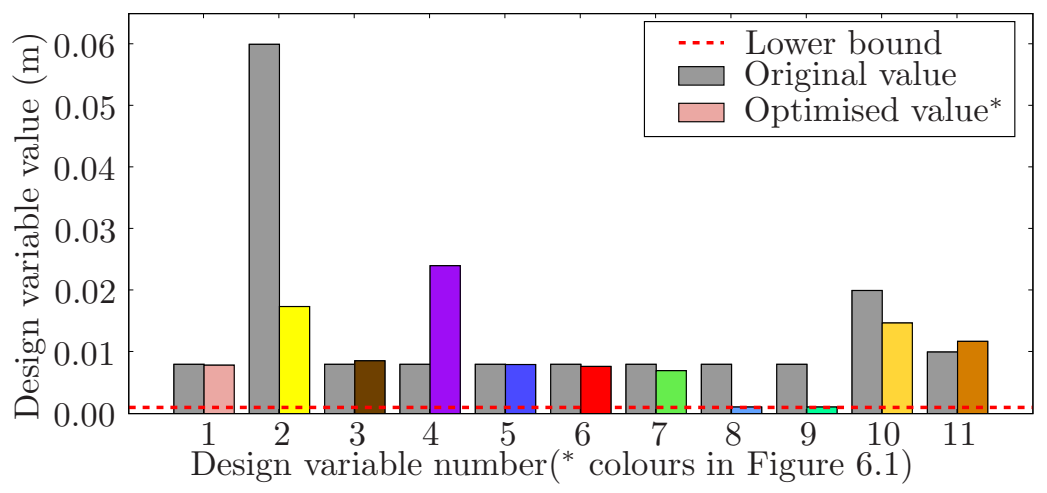

Figure 6.2: Design variables for size optimisation of the original yoke

\subsubsection{Backing structure}

Optimisation of the original backing structure was done by implementing sizeand shape optimisation. The goal was to minimise the mass of the backing structure subject to the constraints in Section 6.1.2. The design variables for the size optimisation are the thicknesses of different parts of the backing structure. In Figure 6.3(a) the colours indicate the different design variables for size optimisation, which were again constrained to be larger than $1 \mathrm{~mm}$. For the shape optimisation, the top taper of all the legs in the backing structure were designed. The domain elements used during the shape optimisation are shown in green in Figure 6.3(b). As shown in Figure 6.3(b) shape optimisation was not implemented on the central part of the backing structure. The shape optimisation design variables for the two vertical legs were linked to ensure that they remain symmetrical after the optimisation. The same were done for the four horizontal legs and the vertical triangles. In total seven size optimisation- and 20 shape optimisation design variables were implemented.

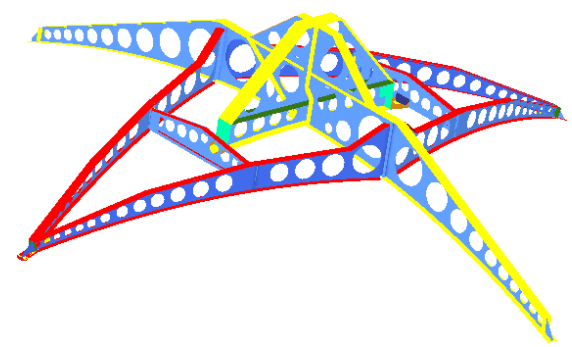

(a) Original backing structure with the colours indicating different properties

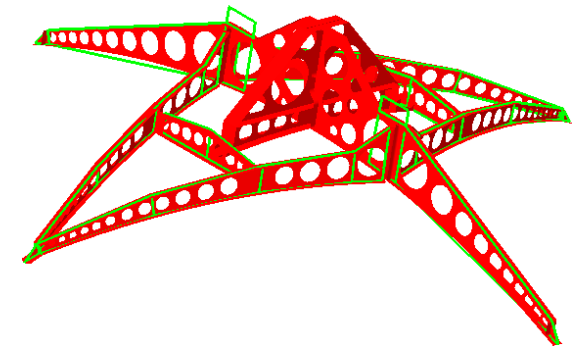

(b) The original backing structure with the shape domains indicated in green

Figure 6.3: The backing structure 
The results obtained from the size- and shape optimisation are shown in Figures 6.4 and 6.5 and Table 6.4. From Figure 6.4, it is clear that design variables 1 and 5 increased substantially, with the remainder of the design variables becoming smaller. These two design variables have the smallest volume in the backing structure, therefore a large increase in their thickness will lead to only a small increase in the total mass. Design variables 1 and 5 are located at the pinned connection between the backing structure and the yoke. This area of the structure is subjected to high loads. An increase in these two design variables will therefore result in a stronger backing structure. Design variable 4 is near its lower bound and it could be omitted from the structure, but it has to be determined if it contributes to the backing structure's stiffness. The lower bound of the design variable should be reduced (to $0.1 \mathrm{~mm}$ ) and optimisation should be repeated. If the design variable reaches the lower bound again, it indicates that its corresponding plate can be removed from the backing structure.

Figure 6.5 displays the change in shape (in magnitude) of the backing structure after shape optimisation. The two vertical legs experienced the largest shape change, because the loads cause large displacements in the $X Z$-plane. The four horizontal legs and two vertical triangles had a small to negligible change in shape. Even though some of the size optimisation design variables increased substantially, the overall mass of the backing structure decreased by $35 \%$ or $865 \mathrm{~kg}$. The optimisation was completed within 7 design cycles with the non-structural constraints within the specified bounds.

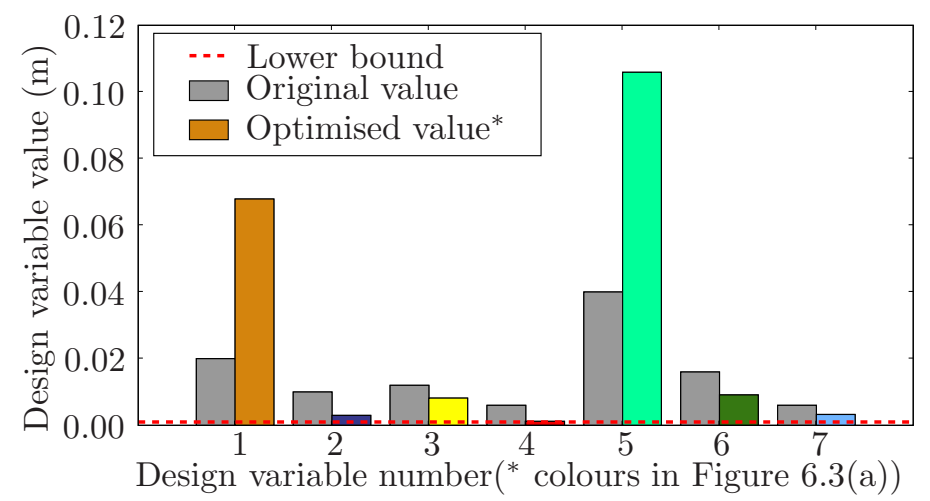

Figure 6.4: Design variables for size optimisation of the original backing structure 


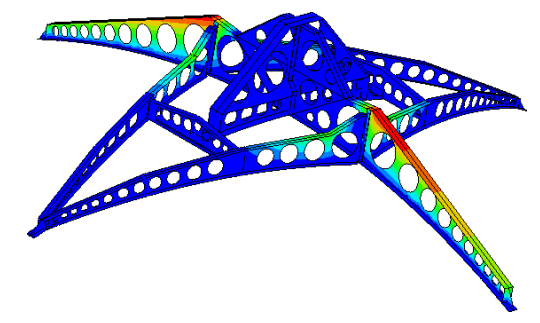

Figure 6.5: The shape changes made to the backing structure (red $=80 \mathrm{~mm}$, green $=48 \mathrm{~mm}$ and blue $=0 \mathrm{~mm}$ )

Table 6.4: Mass results for the original backing structure

\begin{tabular}{lc}
\hline Parameter & Value \\
\hline \hline Number of design cycles & 7 \\
Original mass (kg) & 2459 \\
Optimised mass (kg) & 1594 \\
Mass saved (kg) & 865 \\
Mass saved (\%) & $35 \%$ \\
\hline
\end{tabular}

\subsubsection{FPA arms}

Shape- and size optimisation were implemented to minimise the mass of the FPA arms. Again the constraints of Section 6.1.2 were applied. For the shape optimisation, the bottom and top taper and the width at the top and bottom of the arms were designed. For the size optimisation, the thicknesses of the two vertical plates, two horizontal plates and the triangular plates on the top, were used as design variables for each arm (see Figure 6.6 which indicate the different design variables in colour). The design variables for the bottom and top arms were linked, which will result in the same shape and size for these two arms. The same were done for the right and left arms. In total there are 24 design variables for the shape optimisation and 6 design variables for the size optimisation. Side constraints were put on the design variables which controlled the width and height of the arms for the shape optimisation. This will ensure that the width or height does not become too small, which will lead to a distortion of the finite element mesh. The thicknesses used during size optimisation had a lower bound of $1 \mathrm{~mm}$.

Figures 6.7 and 6.8 and Table 6.5 show the results obtained after size- and shape optimisation were completed on the FPA arms. Figure 6.7 shows a sideand top view of the FPA arms where the red is the original FPA arms and the green, the FPA arms after shape- and size optimisation. Note that the shape changes have been enlarged 5 times to increase visibility (with the largest increase 


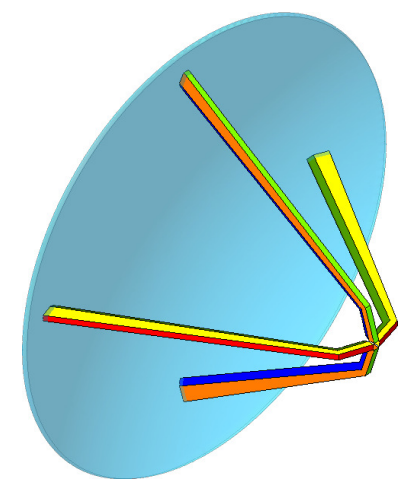

Figure 6.6: Original FPA arms and dish (light blue) with the colours indicating different properties

$57 \mathrm{~mm}$ ). It can be seen that the taper (see Figure 6.7(a)) and the cross sectional width (see Figure 6.7(b)) of the top and bottom arms were enlarged. The shape of the right and left arms stayed basically the same. These shape results can be expected as the gravity and wind loads will be mostly carried by the thicker arms in the $X Z$-plane.

In Figure 6.8 all size optimisation design variables are near the lower bound of $1 \mathrm{~mm}$ except design variables 2 and 3 . The elements corresponding to the design variables which reached the lower bound can not be omitted from the FPA arms. They are still near the thickness of design variables 2 and 3 and will definitely add to the FPA arms' stiffness. Both of the plates controlled by design variables 2 and 3 are part of the top and bottom FPA arms. The largest displacements are in the $X Z$-plane and it makes sense for the optimiser to increase the thickness of these plates to try and minimise the movement of the FPA. After 11 design cycles, the mass of the FPA arms were reduced by $30 \%$, resulting in a mass savings of $290 \mathrm{~kg}$. The non-structural responses were inside their bounds with all the other constraints being met.

Table 6.5: Mass results for the original FPA arms

\begin{tabular}{lc}
\hline Parameter & Value \\
\hline \hline Number of design cycles & 11 \\
Original mass (kg) & 958 \\
Optimised mass $(\mathrm{kg})$ & 668 \\
Mass saved $(\mathrm{kg})$ & 290 \\
Mass saved $(\%)$ & $30 \%$ \\
\hline
\end{tabular}




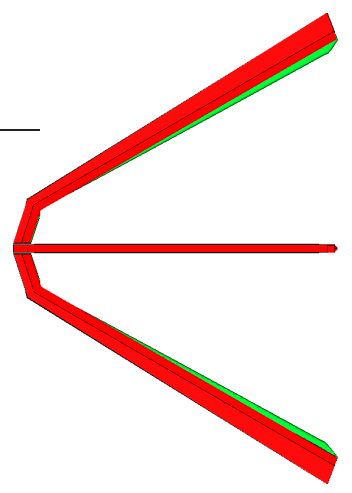

(a) Side view

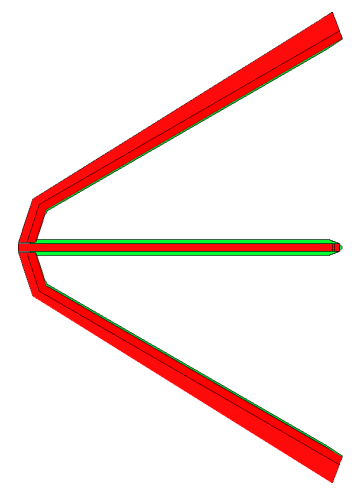

(b) Top view

Figure 6.7: FPA arm's shape before- (red) and after (green) shape optimisation (enlarged five times)

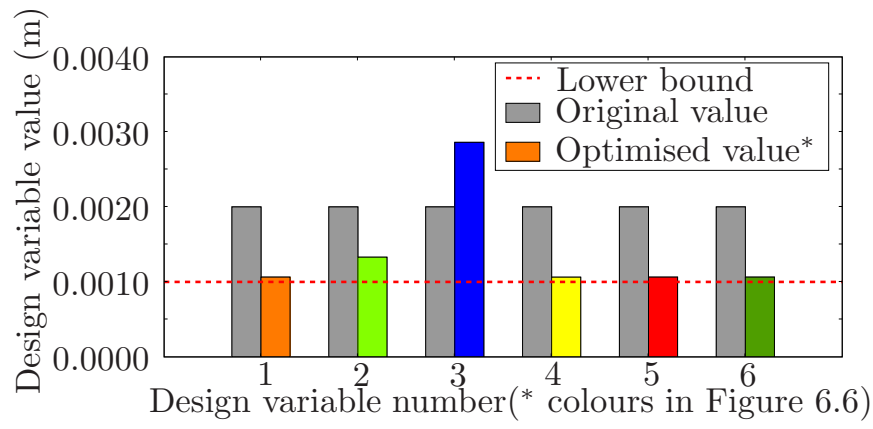

Figure 6.8: Design variables for size optimisation of the original FPA arms

Optimisation of these three existing components showed that the mass of the KAT structure could be substantially reduced without drastic changes to the basic design. Mass minimisation is generally easy to apply to an existing finite element model and is a very powerful tool that can be used during the design process.

\subsubsection{System level optimisation}

The yoke, backing structure and FPA arms of the prototype were optimised together to compare the results obtained from the individual component optimisation with that of a system level optimisation. The same optimisation definition was used as in Sections 6.2.1 - 6.2.3 for the yoke, backing structure and FPA arms.

Table 6.6 shows the mass for the yoke, backing structure and FPA arms of the original prototype, as well as the component optimisation and the system level optimisation. Compared to the prototype, the system level optimisation resulted in a total mass saving of $37 \%$, while the component optimisation resulted only in 
a $26 \%$ mass saving. The system level optimisation thus resulted in an additional $771 \mathrm{~kg}$ mass savings when compared to the component optimisation.

Table 6.6: Comparison between component and system level optimisation

\begin{tabular}{lccc}
\hline Parameters & Prototype & $\begin{array}{c}\text { Component } \\
\text { optimisation }\end{array}$ & $\begin{array}{c}\text { System } \\
\text { optimisation }\end{array}$ \\
\hline \hline Yoke mass (kg) & 3851 & 3404 & 2355 \\
Backing structure mass $(\mathrm{kg})$ & 2459 & 1594 & 1694 \\
FPA arms mass $(\mathrm{kg})$ & 958 & 668 & 546 \\
Total mass saved $(\mathrm{kg})$ & - & 1902 & 2673 \\
Total mass saved $(\%)$ & - & $26 \%$ & $37 \%$ \\
\hline
\end{tabular}

For the system level optimisation, the interaction of components are important. When compared to the component optimisation, the system level optimisation increased the mass of the backing structure to facilitate a lighter yoke and FPA arms, resulting in a lighter overall structure. The backing structure is made heavier and thus stronger, resulting in a stiffer dish. With a stiffer dish, less mass is necessary in the yoke and FPA arms to meet the non-structural constraints. The non-structural responses are almost always active and are the driving force during the optimisation. It is clear that the combination of the optimum components is not the optimum of a structure. To determine the optimum of a structure, all components have to be optimised simultaneously to take the interaction of components into consideration.

In this study, mass minimisation has proven itself to be an effective tool for reducing the mass of the KAT structure. The initial combined mass of the yoke, backing structure and FPA arms is $7268 \mathrm{~kg}$ and it has been lowered to $4595 \mathrm{~kg}$. This is a $2673 \mathrm{~kg}$ reduction in steel which will definitely lower the KAT material costs by a considerable amount.

\subsection{Concept generation}

This section will investigate the use of optimisation for generating new designs. The backing structure and yoke from Sections 6.2.2 and 6.2.1 will be considered. Firstly, topology- and size optimisation will be applied to the backing structure to generate two new concepts for it. Thereafter, two concepts for the yoke will be generated by applying size optimisation.

The mass of the original backing structure, after manufacturing, does not provide an accurate representation of its material costs. More raw material is ini- 
tially required for the backing structure compared to the material of the finalised product, since the final product contains a large number of holes. Therefore, one has to consider the raw material, before the holes are cut, to obtain the actual material costs. In the following sections two new concepts will be generated for the backing structure, where the focus will be on minimising the raw material costs and not necessarily the mass.

Topology optimisation will be implemented for the first concept of the backing structure, to identify the parts which contributes the most to the backing structure's stiffness. For this concept, holes will be kept in the parts which has the least contribution to the backing structure's stiffness. The parts with the largest stiffness contribution will contain no holes. This procedure will identify the optimal hole location in the backing structure. Thereafter, size optimisation will be used to find the optimal plate thicknesses.

The second concept that will be generated for the backing structure will contain no holes. This concept will look identical to the original backing structure, but all holes will be removed and filled with material. Again, size optimisation will be utilised to determine the concept's optimal plate thicknesses.

\subsubsection{Topology optimisation}

The rationale of this concept is to find the optimal hole locations in the backing structure. It is a non-trivial task to find these parameters, but topology optimisation can be implemented to aid in this task. The goal of this section will be to determine the optimal location of holes (if any) in the backing structure. Thereafter, size optimisation will be applied to find the optimal plate thicknesses for the specified hole locations.

Topology optimisation will be applied to the web of the backing structure (the plate thicknesses are identical to that of the original non-optimised backing structure). The optimiser will make optimum use of the allowable mass fraction, indicating which parts of the structure contributes the most to the stiffness. Holes will be placed in areas that contribute the least to the stiffness.

In order to apply topology optimisation, the backing structure should not contain any holes. The backing structure was re-meshed to fill all the holes. See Figures 6.9 for an illustration of the backing structure without holes.

The objective function for the topology optimisation was to minimise the strain energy. The backing structure was constrained to be symmetrical about the $X Y$ - and $X Z$-planes. This will ensure that the four horizontal legs are identical as well as the two vertical legs. Four topology optimisations were executed, each 


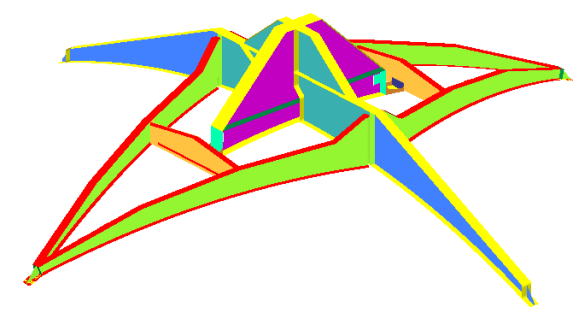

Figure 6.9: The backing structure without holes

time with a different mass fraction constraint. Mass fractions of: $0.2,0.3,0.4$ and 0.5 were used. Figure 6.10 displays the retained material (blue) and the removed material (yellow) for the four different mass fraction cases.

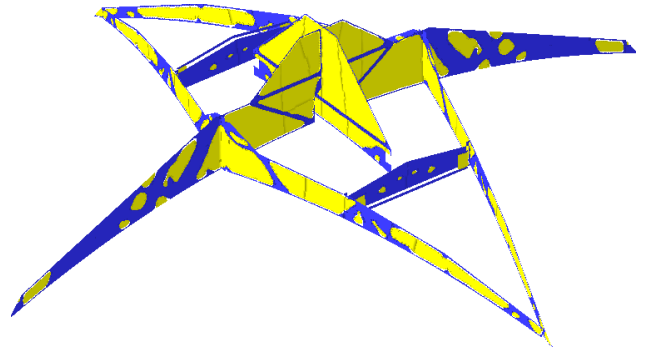

(a) Mass fraction: 0.2

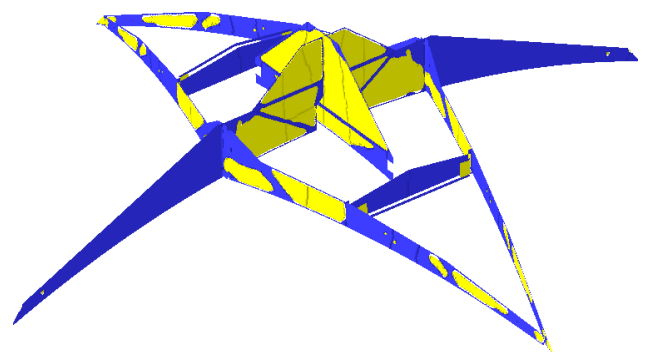

(c) Mass fraction: 0.4

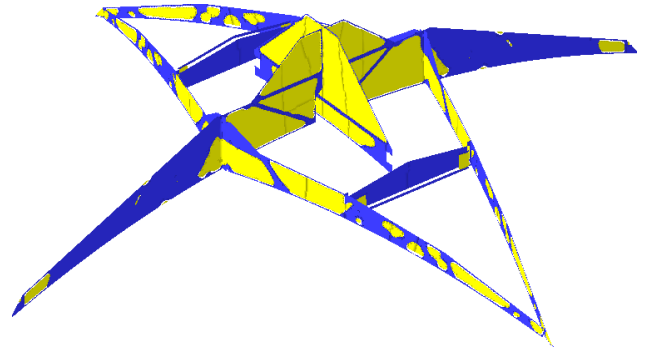

(b) Mass fraction: 0.3

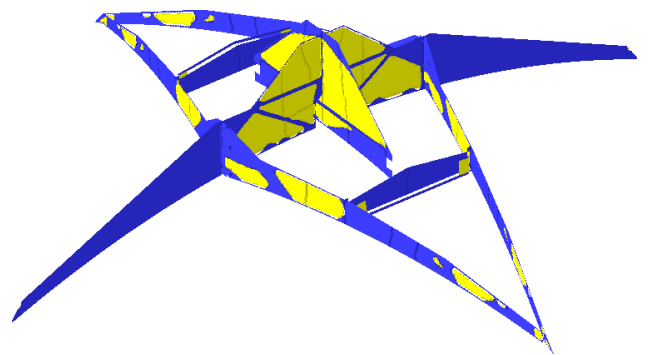

(d) Mass fraction: 0.5

Figure 6.10: Retained material (blue) and removed material (yellow) for the four cases of topology optimisation

The results obtained from the topology optimisation give some insight into the best hole locations. From Figure 6.10 it can clearly be seen that the two vertical legs and the two vertical triangles contribute most to the backing structure's stiffness. Very little material are removed from these parts for all four cases of the mass fraction constraints. The four horizontal legs contribute less to the stiffness as some material is removed from it during the topology optimisation. The set of plates in the middle of the backing structure have almost no contribution to the stiffness. Nearly all material were removed from these two plates.

The geometry obtained from the topology optimisation can not directly be converted into a structure. Topology optimisation only provides an indication 
of the parts of the structure which contribute the most to its stiffness. A new concept (referred to as concept B2) was generated for the backing structure, based on the observations made from the topology optimisation results. It was decided to remove all holes from the two vertical legs as well as the two vertical triangles of the backing structure. The rest of the backing structure remained the same, with the hole locations identical to that of the prototype. The new concept for the backing structure (concept B2) is displayed in Figure 6.11.

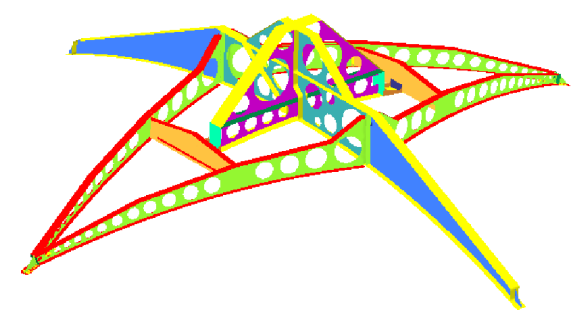

Figure 6.11: Backing structure with some of the holes removed (Concept B2)

\subsubsection{Backing structure - Some holes removed}

The optimal distribution of holes has been determined for concept B2. The next step will be to determine the concept's optimal plate thicknesses. This will be accomplished by applying size optimisation.

The size optimisation was setup to minimise the mass subject to the same constraints as given in Section 6.1.2. Again the design variables will be the plate thicknesses which were constrained with a lower bound of $1 \mathrm{~mm}$. The different design variables are indicated in colour in Figure 6.11. Note that the web was divided into five different properties to give the optimiser more freedom during the optimisation. For shape- and size optimisation of the original backing structure, the web only consisted of one design variable (see Section 6.2.2), since the goal was to change the design as little as possible.

The results obtained from the size optimisation are shown in Figure 6.12 and Table 6.7. From Figure 6.12 it is clear that design variable 5 increased substantially. This design variable also had a large increase for size- and shape optimisation of the original backing structure. The same reason can be ascribed for its large increase, as presented in Section 6.2.2. Design variables 2, 4, 7 and 9 approached the lower bound of $1 \mathrm{~mm}$. These design variables could be omitted from the structure, but it has to be determined if these plates contribute to the backing structure's stiffness. The lower bounds of these design variables should 
be reduced (to $0.1 \mathrm{~mm}$ ) and optimisation should be repeated. If the design variables reaches the lower bound again, it indicates that their corresponding plates can be removed from the backing structure. The optimisation was completed within 10 design cycles and all constraints were inside the specified bounds. The optimised raw material needed for this concept is $1028 \mathrm{~kg}$ less, in comparison with the raw material needed for the original backing structure. That is a $35 \%$ decrease in raw material mass.

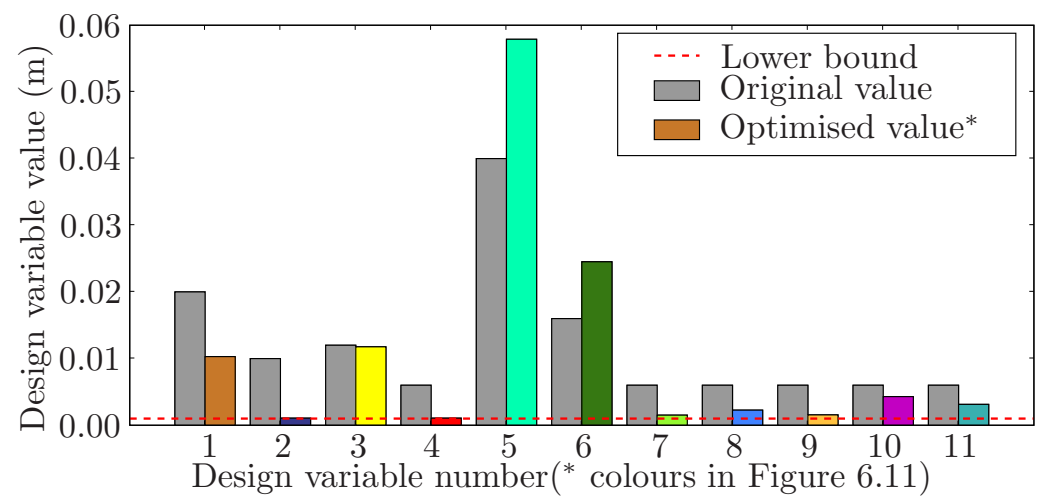

Figure 6.12: Design variables for size optimisation of the backing structure (Concept B2)

Table 6.7: Mass results for the backing structure (Concept B2)

\begin{tabular}{lc}
\hline Parameter & Value \\
\hline \hline Number of design cycles & 10 \\
Optimised raw material $(\mathrm{kg})$ & 1884 \\
Raw material saved compared to B0 $(\mathrm{kg})$ & 1028 \\
Raw material saved compared to B0 $(\%)$ & $35 \%$ \\
\hline
\end{tabular}

Concept B2 can be refined which could lead to an even better result. Figure 6.13 shows concept B2 (see Figure 6.11) which had been overlayed with the retained material from the topology optimisation with a mass fraction constraint of 0.3 (see Figure 6.10(b)). A possible improvement is to keep concept B2 as it is and additionally close the holes which are encircled in Figure 6.13. This could result in a lighter design, as the suggested design are closer to the topology optimisation results. This was not investigated, because of a restriction in time.

The utilisation of topology- and size optimisation produced a concept for the backing structure which will have cheaper raw material costs compared to the 


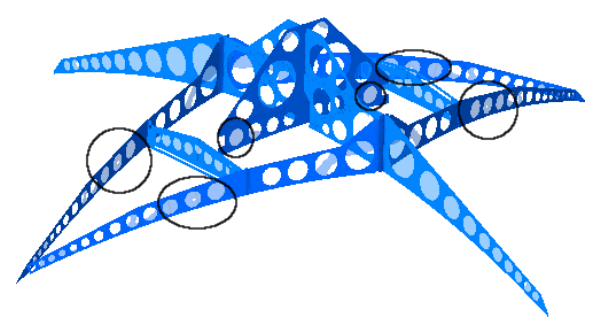

Figure 6.13: Proposed improvement on Concept B2

original backing structure. This is a non-trivial task which will not easily be accomplished without the use of structural optimisation.

\subsubsection{Backing structure - All holes removed}

Another possible concept for the backing structure is to remove all the holes in the web (referred to as concept B3), as shown in Figure 6.9. Size optimisation will most likely produce thinner plates for this concept, resulting in less required raw material in comparison with the original backing structure. The manufacturing cost are also lowered as laser cutting is not necessary for cutting holes. Note that the raw material necessary for this concept is equivalent to its optimised mass as no holes are present. Size optimisation was applied to this concept to determine the optimal plate thicknesses.

The objective function for size optimisation of the backing structure was mass minimisation. The design variables are again the plate thicknesses where the different design variables are indicated in colour in Figure 6.9. A lower bound of $1 \mathrm{~mm}$ has been assigned to limit all design variables. The constraints induced on the backing structure are given in Section 6.1.2.

The results for size optimisation on concept B3 can be seen in Figure 6.14 and Table 6.8. There is a large increase in design variable 5 and a considerable decrease in design variable 1 . Most of the design variables experienced a small change compared to their initial values, except design variables 2, 4 and 7, which are near the lower bound. These design variables could be omitted from the structure, but it has to be determined if these plates contribute to the backing structure's stiffness. The lower bounds of these design variables should be reduced (to $0.1 \mathrm{~mm}$ ) and optimisation should be repeated. If the design variables reaches the lower bound again, it indicates that their corresponding plates can be removed from the backing structure. After 11 design cycles the raw material of the backing structure decreased by $1296 \mathrm{~kg}$, which is a $45 \%$ saving in raw material compared to the original backing structure. 


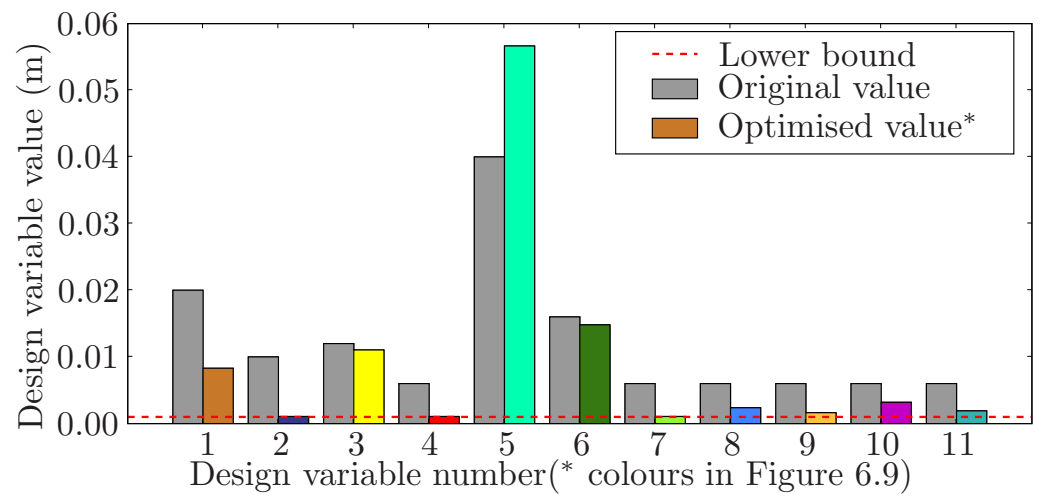

Figure 6.14: Design variables for size optimisation of the backing structure (Concept B3)

Table 6.8: Mass results for the backing structure (Concept B3)

\begin{tabular}{lc}
\hline Parameter & Value \\
\hline \hline Number of design cycles & 11 \\
Optimised raw material $(\mathrm{kg})$ & 1616 \\
Raw material saved compared to B0 $(\mathrm{kg})$ & 1296 \\
Raw material saved compared to B0 $(\%)$ & $45 \%$ \\
\hline
\end{tabular}

Structural optimisation has made it possible to generate a concept for the backing structure which is far better than the original backing structure. It requires less raw material and contains no holes which reduces the material- and manufacturing costs.

\subsubsection{Yoke - Outer shell}

All the internal plates for the yoke were removed to generate a new concept for the yoke (referred to as concept Y2) which is shown in Figure 6.15(a). The rationale is a new concept that is much cheaper to manufacture. Size optimisation was applied to determine the optimum thickness of the outer shell. This concept will show if the internal plates are necessary. The manufacturing cost will decrease by a large amount if the internal plates are removed, since a large amount of welding will be eliminated. The constraints and side constraints that were applied are the same as with the original yoke in Section 6.2.1.

Table 6.9 shows the results obtained after size optimisation of this concept. After 27 design cycles the optimisation converged to an infeasible design with a maximum constraint violation of $33 \%$. The least infeasible point had a mass of $3315 \mathrm{~kg}$ and a maximum constraint violation of $22 \%$. 
The bounds on the non-structural constraints are too high for this specific layout of the yoke. It is impossible to overcome the constraints by only altering the plate thicknesses. The structural layout of the yoke must be modified to meet the constraints (see a further discussion about this subject in Section 6.5). Therefore one can conclude that the internal plates in the yoke are necessary in order to meet the design constraints.

Table 6.9: A comparison of the initial- and least infeasible design point for the yoke (Concept Y2)

\begin{tabular}{lccc}
\hline Design & $\begin{array}{c}\text { Design } \\
\text { cycles }\end{array}$ & Mass (kg) & $\begin{array}{c}\text { Constraint } \\
\text { violation (\%) }\end{array}$ \\
\hline \hline Initial & 0 & 2779 & $154 \%$ \\
Least infeasible & 8 & 3315 & $22 \%$ \\
\hline
\end{tabular}

\subsubsection{Yoke - Added stiffness}

The previous concept for the yoke resulted in an infeasible design when all the internal plates were removed. Another concept (referred to as concept Y3) was considered where two of the internal plates were again added (see Figure 6.15(b)), to increase the stiffness of the yoke and hopefully result in a feasible design. These two internal plates will add to the manufacturing cost, but it will still be much cheaper to manufacture than the original concept. The objective function was mass reduction and the design variables were the thickness values of the five chosen plates. The same constraints and side constraints were used as in Section 6.2.1.

Figure 6.16 and Table 6.10 show the results obtained after size optimisation. Design variables 1, 2 and 5 increased, with design variables 2 and 5 experiencing the largest increase. These plates are very thick and it could be a problem to actually manufacture them. Design variables 3 and 4 decreased and are very near the lower bound. This indicates that the added plates did not contribute to the stiffness of the yoke, but they did aid in finding a feasible design. Optimisation was completed in 29 design cycles with a $117 \%$ increase in mass compared to the original yoke. This concept was infeasible at the start of the optimisation and the optimiser added mass, to overcome the infeasibility, resulting in a heavier design. All constraints including the non-structural constraints are met. 


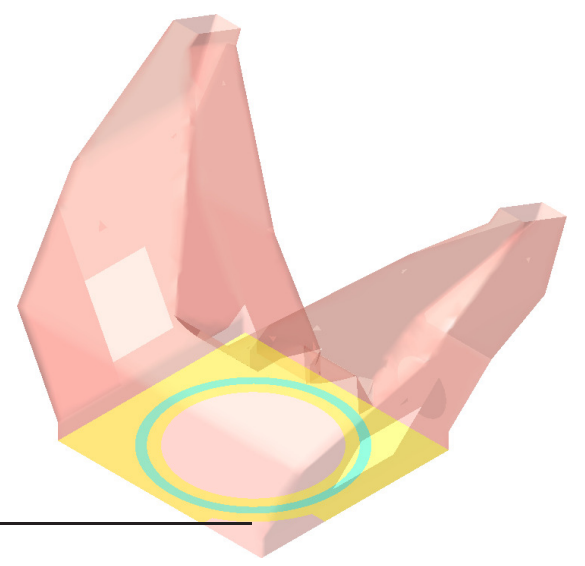

(a) Yoke with the internal plates removed (Concept Y2)

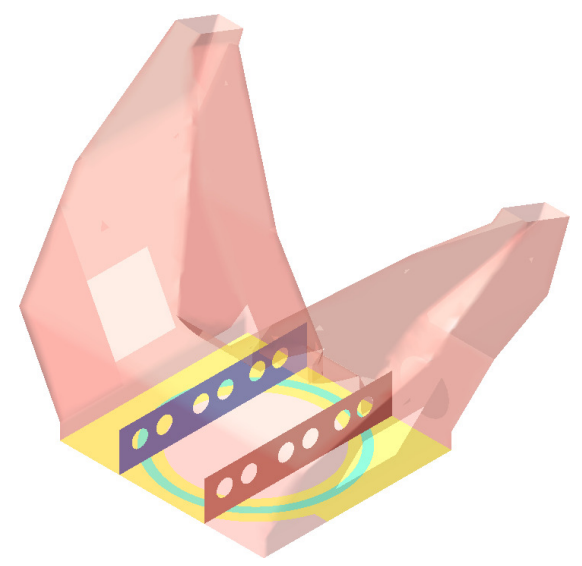

(b) Yoke with only two internal plates (Concept Y3)

Figure 6.15: Two new concepts for the yoke

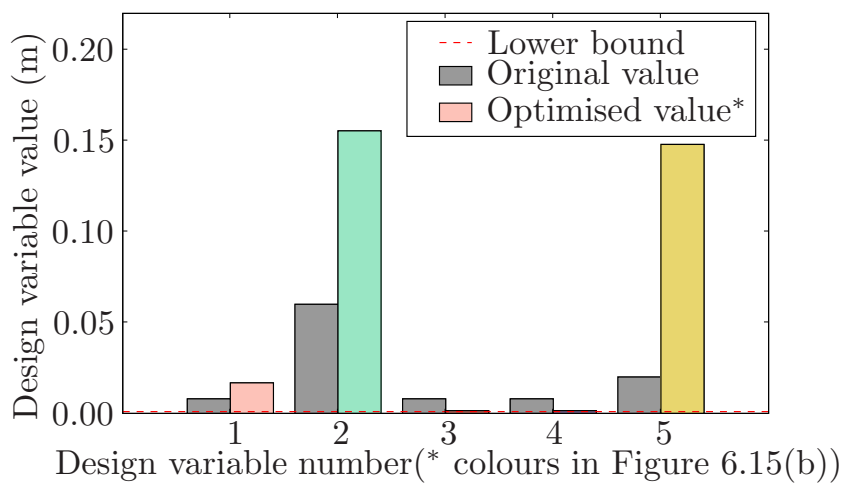

Figure 6.16: Design variables for size optimisation of the yoke (Concept Y3)

Table 6.10: Mass results for the yoke (Concept Y3)

\begin{tabular}{lc}
\hline Parameter & Value \\
\hline \hline Number of design cycles & 29 \\
Original mass (kg) & 2894 \\
Optimised mass (kg) & 8362 \\
Mass added compared to Y0 (kg) & $\mathbf{4 5 1 1}$ \\
Mass added compared to Y0 (\%) & $117 \%$ \\
\hline
\end{tabular}

In this section, two concepts were generated for the yoke and backing structure with the help of structural optimisation. These concepts are designed at their optimum and are now ready to be compared with each other. 


\subsection{Concept evaluation}

During concept evaluation, the wrong concept can easily be chosen if all concepts are not optimised before the concept evaluation phase. The utilisation of structural optimisation can prevent this, by finding the optimum design for each concept.

This section will investigate the use of optimisation for comparing concept designs. The concepts that were generated for the backing structure and yoke in this study, will be compared with each other.

\subsubsection{Backing structure}

Four different concepts have been identified for the backing structure. These concepts include the original backing structure (B0), the original size optimised backing structure (B1), the optimised backing structure with some holes removed (B2) and the optimised backing structure where all holes have been removed (B3). The optimisation of concepts B2 and B3 are discussed in Sections 6.3.2 and 6.3.3. For concept B1 only size optimisation has been implemented. The same procedure has been followed as in Section 6.2.2 with the only difference being the omition of shape optimisation from the optimisation definition. A comparison of concepts B0, B1, B2 and B3 are shown in Table 6.11.

Table 6.11: Comparison between the different concepts for the backing structure

\begin{tabular}{lcccc}
\hline Parameters & B0 & B1 & B2 & B3 \\
\hline \hline Non-optimised raw material $^{a}(\mathrm{~kg})$ & 2912 & 2912 & 2893 & 4004 \\
Optimised raw material $^{b}(\mathrm{~kg})$ & - & 1974 & 1884 & 1616 \\
Optimised post manufactured material $^{c}(\mathrm{~kg})$ & 2459 & 1738 & 1734 & 1616 \\
Raw material saved compared to B0 $(\mathrm{kg})$ & - & 938 & 1028 & 1296 \\
Raw material saved compared to B0 $(\%)$ & - & $32 \%$ & $35 \%$ & $45 \%$ \\
\hline
\end{tabular}

\footnotetext{
${ }^{a}$ Raw material with the thicknesses of the original backing structure (no holes)

${ }^{b}$ Raw material with optimised thicknesses (no holes)

${ }^{c}$ Material with optimised thicknesses including holes
}

From Table 6.11 it is clear that three backing structure concepts have been generated with structural optimisation which requires less raw material than the original backing structure. Size optimisation of concept B3 lead to a reduction of $45 \%$ in raw material which is the largest decrease in raw material of all the concepts. This shows that the insertion of holes into a concept, might make it lighter, but it will not necessarily lead to a cheaper concept. The manufactured 
concept B2 is only $4 \mathrm{~kg}$ lighter than the manufactured concept B1, but it requires $90 \mathrm{~kg}$ less raw material. This indicates that the raw material are the deciding factor in determining the best concept and not the post manufactured mass.

The importance of evaluating concepts at their optimum became evident during comparison of the different backing structure concepts. The non-optimised concept B3 looks very poor in comparison with concept B0 as it requires much more raw material. Optimisation of these two concepts produces the opposite as the optimised concept B3 requires less raw material in comparison with an optimised concept B0.

Many times a trade-off must be made between the material costs and the manufacturing costs in order to decide which concept is the best. The material costs are directly related to the mass and can therefore be taken into account during the optimisation process. The heavier the structure, the more expensive the material costs will become. Unfortunately, manufacturing costs can not directly be taken into consideration during the optimisation process. Sometimes a study has to be undertaken to determine the manufacturing costs of the concepts in order to decide on the best concept. For this case it was not necessary. Concept B1 will be more expensive to manufacture than concept B2 as it contains more holes that has to be laser cut. Concept B2 is therefore better than concept B1, since it requires less raw material and laser cutting. The best choice, however, will be concept B3 as it consists of no holes and requires no laser cutting which will result in the least manufacturing- and raw material costs.

\subsubsection{Yoke}

Four different concepts were generated for the yoke. The original non-optimised yoke (Y0), the original optimised yoke (Y1), the optimised outer shell yoke (Y2) and the optimised stiffened yoke (Y3). The optimisation of concepts Y1, Y2 and Y3 are discussed in Sections 6.2.1, 6.3.4 and 6.3.5 respectively. A comparison of concepts Y0, Y1 and Y3 are shown in Table 6.12. Note that concept Y2 is not included in Table 6.12 as it did not result in a feasible design.

Table 6.12: Comparison between the different concepts for the yoke

\begin{tabular}{lccc}
\hline Parameters & Y0 & Y1 & Y3 \\
\hline \hline Original mass $(\mathrm{kg})$ & 3851 & 3851 & 2894 \\
Optimised mass $(\mathrm{kg})$ & - & 3404 & 8362 \\
Mass change to Y0 $(\%)$ & - & $-12 \%$ & $+117 \%$ \\
\hline
\end{tabular}


Concept Y2 will be cheaper to manufacture than all the other concepts (less welding will be necessary), but has to be eliminated as it does not result in a feasible design. Concept Y1 is lighter compared to concept Y3, but the welding cost of concept Y1 will be more expensive than concept Y3. However, it will not be practically viable to manufacture thick plates (represented by design variables 2 and 5 in Figure 6.16) of concept Y3, which are in excess of a $100 \mathrm{~mm}$. Therefore it will be cheaper to weld the extra plates for concept Y1 than to bend and weld concept Y3.

Again the material- and manufacturing costs have to be brought into consideration. Concept Y1 are considerably lighter than concept Y3. The manufacturing costs of concept Y3 will also be higher than that of concept Y1, resulting in concept Y1 being the better choice.

During the concept evaluation of the backing structure and the yoke it was evident that it is not a trivial task to compare different concepts with each other. Various factors have to be considered during the concept evaluation, including design feasibility, structural mass, raw material costs and manufacturing costs. Structural optimisation aided by provding optimal designs which ensures that the correct concept can be selected.

\subsection{Design for structural feasibility}

Often during the structural design process, one may find that a design is infeasible. Due to the complexity of some designs it may be extremely difficult to determine what changes are required to satisfy the design requirements. The extent to which the design variables should be changed and the effect that it will have on the structure, is not an easy assignment.

Structural optimisation provides a solution to the above mentioned problem. It can alter the design variables to overcome a constraint violation, changing an infeasible design into a feasible design. One way of finding a feasible design is to setup the optimisation problem as mass minimisation. If a design is initially infeasible, the first goal of the optimiser will be to find a feasible design (see Section 2.1.4). Only when a feasible point has been found, will the optimiser try to reduce the objective function.

The above explained phenomenon has been encountered earlier in this study. The changes made to the backing structure (see Sections 6.3.2 and 6.3.3) and yoke (see Section 6.3.5) resulted in initially infeasible designs. After these designs have been optimised, the objective function either decreased or increased, but a feasible 
design was found.

An alternative to the above mentioned procedure is to apply the $\beta$-method [36] which can be summarised as follow:

Minimise:

Subject to:

$$
g_{i}(\mathbf{X}) \leq \beta \quad i=1,2, \ldots, p
$$

where

$$
\begin{gathered}
\mathbf{X}=X_{1}, X_{2}, \ldots, X_{l}, \beta \\
X_{k}^{l} \leq X_{k} \leq X_{k}^{u} \quad k=1,2, \ldots, l \\
\beta \geq 0
\end{gathered}
$$

The objective function is to minimise an artificial design variable, $\beta$ (6.5.1). It is subject to the normalised constraints which should be less than or equal to $\beta$ (6.5.2). One prerequisite is that $\beta$ should be larger than or equal to zero (6.5.5). The rest of the design variables (6.5.3) and their side constraints (6.5.4) remain the same as the standard optimisation problem (see Section 2.1.3).

This method can be applied to change an infeasible design into a feasible design by minimising the maximum constraint violation. To visualise this, consider Figure 6.17. The bar graphs signifies the constraint violations (where the red bar represents the maximum constraint violation) that exist in the optimisation problem. The current value of $\beta$ is indicated by the red line. The optimiser will try to make $\beta$ as small as possible (shift the red line downwards), which can only be achieved by reducing the maximum constraint violation.

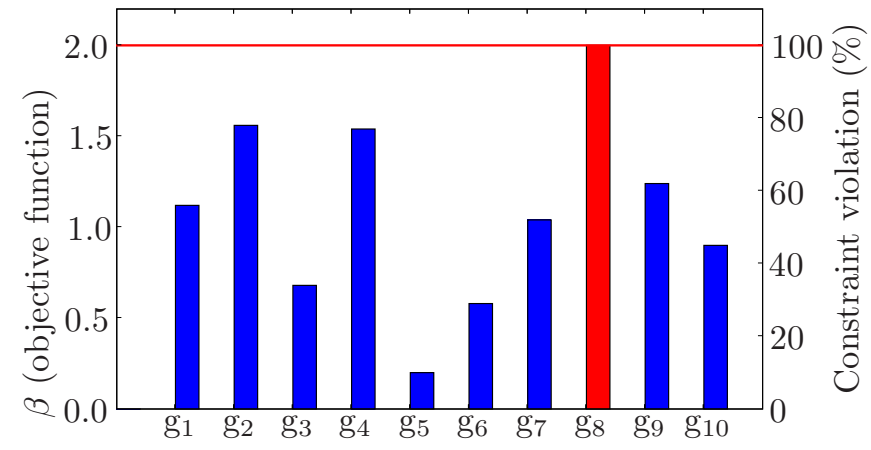

Figure 6.17: Visualisation of minimising the maximum constraint violation with the $\beta$-method 
To illustrate the design for structural feasibility, the $\beta$-method can be implemented on the KAT antenna. Some of the KAT antenna's non-structural responses are larger than the specified design requirements (see Table 6.2). Therefore, the KAT prototype design is infeasible when evaluated against the original design requirements.

Size- and shape optimisation were implemented simultaneously on the yoke, backing structure and FPA arms to try and find a feasible design for the KAT. The optimisation problem was defined according to the $\beta$-method, where $\beta$ was added as an artificial design variable according to (6.5.5). The objective function was to minimise $\beta$, subject to the constraints in Section 6.1.2. The original constraints for all violated non-structural responses (there are seven in total) were replaced with constraints that have the form shown in (6.5.2). All non-structural constraints were limited to be no larger than the design requirements.

During the optimisation process, the optimiser could not find a feasible design. However, it lowered the constraint violation from $177 \%$ to $163 \%$. The least infeasible point was compared with the initial design of the KAT. The results are displayed in Table 6.13. The least infeasible design point was found after 6 design cycles and the objective function increased with $299 \%$ or $21710 \mathrm{~kg}$.

Table 6.13: A comparison of the initial- and least infeasible design points for the KAT

\begin{tabular}{lccc}
\hline Design & $\begin{array}{c}\text { Design } \\
\text { cycles }\end{array}$ & Mass (kg) & $\begin{array}{c}\text { Max. Constraint } \\
\text { violation }(\%)\end{array}$ \\
\hline \hline Initial & 0 & 7266 & $177 \%$ \\
Least infeasible & 6 & 28976 & $163 \%$ \\
\hline
\end{tabular}

Table 6.14 shows a comparison between the initial- and least infeasible, violated non-structural responses (the dish surface errors and relative FPA rotations were not violated and are not displayed). Only one response, the relative FPA $X$-displacement for loadcase $\mathrm{C}$, is not violated initially. At the least infeasible design point, the maximum constraint violation has been reduced and only two responses (printed in bold) are far away from the design requirement. The rest of the responses are within $36 \%$ from the design requirement. This shows that although the optimiser could not find a feasible design, it was at least capable of lowering the constraint violations. Note that none of the structural responses have been violated during the optimisation.

Upon first glance of the results, one would think that structural optimisation could not provide a feasible design, therefore discarding the argument that it can 
Table 6.14: A comparison of the initial- and least infeasible non-structural responses

\begin{tabular}{llccc}
\hline $\begin{array}{l}\text { Load- } \\
\text { case }\end{array}$ & $\begin{array}{l}\text { Non-structural } \\
\text { responses }\end{array}$ & $\begin{array}{c}\text { Design } \\
\text { requirement }\end{array}$ & $\begin{array}{c}\text { Initial } \\
\text { response }\end{array}$ & $\begin{array}{c}\text { Least } \\
\text { infeasible } \\
\text { response }\end{array}$ \\
\hline \hline $\mathrm{A}$ & $X$-displacement $(\mathrm{mm})$ & 10.0 & -14.1 & -13.3 \\
& $Y Z$-plane-displacement $(\mathrm{mm})$ & 10.0 & 16.0 & 12.1 \\
\hline \hline $\mathrm{B}$ & $X$-displacement $(\mathrm{mm})$ & 10.0 & 27.7 & $\mathbf{2 6 . 3}$ \\
& $Y Z$-plane-displacement $(\mathrm{mm})$ & 10.0 & 15.4 & 11.4 \\
\hline \hline $\mathrm{C}$ & $X$-displacement $(\mathrm{mm})$ & 10.0 & 6.2 & 5.7 \\
& $Y Z$-plane-displacement $(\mathrm{mm})$ & 10.0 & 14.8 & 10.7 \\
\hline \hline $\mathrm{D}$ & $X$-displacement $(\mathrm{mm})$ & 10.0 & 19.6 & $\mathbf{1 8 . 4}$ \\
& $Y Z$-plane-displacement $(\mathrm{mm})$ & 10.0 & 17.9 & 13.6 \\
\hline
\end{tabular}

be used to ensure a feasible design. However, this is not the case, since it signifies that the constraint bound is too tight and the optimiser can not find a feasible design for the current structural design. The constraint can only be overcome with a conceptual change to the structural design. The generated yoke concepts are a good example of this phenomenon. The optimiser could not find a feasible design for the outer shell yoke (concept Y2). Two plates had to be added to concept Y2 (resulting in concept Y3) to increase the stiffness, which resulted in a feasible design.

Structural optimisation can be implemented to convert an infeasible design into a feasible design. The only time when it can not find a feasible design, is when the constraint bound is too firm. Therefore, structural optimisation can be used effectively by the designer to ensure a suitable design which meets a specified set of constraints. 


\section{Chapter 7}

\section{Conclusion}

In closing, a few suggestions are made concerning future work on the optimisation of the KAT antenna. Finally, the most important conclusions of this study will be presented.

\subsection{Future work}

Although the work presented in this study indicates that structural optimisation can be used to obtain significant mass savings, additional work is required to obtain a final design for the KAT antenna that is ready for manufacturing. This section provides suggestions for future work on the KAT design.

The dish of the KAT was only modelled at a zero pitch- and azimuth angle. The designs presented in this study are only viable for this case and might become infeasible if these angles are varied. Further investigation at other pitch- and azimuth angles is therefore necessary.

A limitation on the optimisations presented is that the design variables are continuous. If the design variables are for example plate thickness values (which are often the case), it will be impossible to find the optimum plate thickness in industry. Plates are produced only at a finite set of thickness values. In reality a thicker plate has to be selected many times, compared to the plate thickness at the optimum. This will result in a heavier structure as was determined by the optimisation. The solution for this problem would be to implement discrete optimisation. For discrete optimisation, only a discrete set of predefined values can be assigned to a design variable.

The effect of fatigue on the KAT antenna was not investigated at all. A thorough investigation must be done to ensure that the proposed designs do not fail due to fatigue. Less mass would perhaps be saved if one consider the effect 
of fatigue on the presented designs.

Manufacturing costs were not directly considered during the optimisation processes. An interesting addition to the optimisation problem would be to add constraints based on the manufacturing costs. This could be implemented by utilising an external program which calculates the manufacturing costs for a proposed design.

During the optimisation, some of the plate thicknesses were near the lower bound of $1 \mathrm{~mm}$. Plate thicknesses of $1 \mathrm{~mm}$ can cause buckling and buckling constraints could be added during optimisation to prevent buckling (see [15; 37]). Therefore a thorough buckling study should be undertaken to ensure that the plates do not buckle.

\subsection{Conclusion}

In conclusion, a brief literature study of numerical design optimisation with the focus on structural optimisation, has been presented. The KAT antenna and its high manufacturing cost has been introduced. The conversion of the ANSYS finite element model to a MSC.NASTRAN finite element model and the difficulties encountered have been discussed. The GENESIS finite element model did not reproduce exactly the same results as the ANSYS finite element model. However, the results produced by the GENESIS finite element model are similar compared to the ANSYS finite element model's results, indicating that it is a representative model which can be utilised to illustrate structural optimisation applications. The structure and operation of an external program, for the calculation of non-structural responses, was discussed. A two level parallelisation method was developed and implemented, which resulted in a reduction in computational time of between $58 \%$ and $93 \%$ (depending on the number of design variables).

Four different applications of structural optimisation have been discussed and illustrated using a real-life engineering problem. The applications considered, were mass minimisation, concept generation, concept evaluation and design for structural feasibility.

Mass minimisation of the KAT components separately, lead to a mass reduction of $1902 \mathrm{~kg}$. When all the components were optimised simultaneously, a total mass of $2673 \mathrm{~kg}$ was saved. This showed that the sum of the optimum components is not necessarily the optimum of the whole structure. The interaction of the components during the optimisation has to be considered. It has been shown 
that small changes made to an existing structural design could lead to a large reduction in mass and ultimately cost.

For the concept generation, three concepts were developed for the backing structure and the yoke by implementing size- and/or topology optimisation. All three the backing structure concepts experienced a decrease in raw material of between $32 \%$ and $45 \%$ in comparison with the original backing structure. This will not easily be accomplished without the use of structural optimisation. Structural optimisation is a powerful design tool for generating concepts, even if nonstructural responses have to be considered. Many times there is a difference between the amount of raw material needed to manufacture a design and the actual amount of material after manufacturing. If this is the case, the material costs will only be minimised if the amount of raw material is decreased.

Various factors must be considered during concept evaluation, including design feasibility, structural mass, raw material costs and manufacturing costs. This makes concept evaluation a non-trivial task. Two components of the KAT, namely the backing structure and the yoke were investigated during concept evaluation. Three concepts were evaluated for each component where each concept was designed at its optimum with the help of structural optimisation. The optimised prototype yoke and the optimised backing structure containing no holes, were found to be the best concepts. It would have been pointless to compare these concepts with each other if each concept was not designed at its optimum.

The material costs are not always the deciding factor in choosing a concept. Many times, a trade-off between the material- and manufacturing costs will reveal the cheapest concept. Unfortunately, manufacturing costs are not directly accounted for during the optimisation process and a lighter structure is not always cheaper to produce. However, careful application of structural optimisation techniques, combined with sound engineering judgement, provides a powerful tool for investigating possible cost savings.

An important application of structural optimisation is to convert an infeasible design into a feasible design. Various examples have been provided, indicating that the optimiser overcame the initial constraint violation, often times by increasing the objective function. Optimisation of the KAT, constrained at the specified design requirements, resulted in an infeasible design, indicating that the constraint bound is too firm for the current structural design. The only way to overcome the constraint violation is to consider a new structural design.

Numerical design optimisation provides one way of reducing manufacturingand material costs of a structure. If a finite element model is available, it is generally straight-forward to apply structural optimisation to it. In this study, 
structural optimisation has been applied to the finite element model of an actual radio telescope of which a full scale prototype was built. It was shown that $2673 \mathrm{~kg}$ steel can be saved for the KAT antenna structure without drastic changes to the basic design. If it is considered that a large number of these antennas will be manufactured, even a small cost saving for one antenna will result in a large cost saving for the whole project. 


\section{Bibliography}

[1] Mundt, C. and Quinn, G.: Test-analysis correlation with design optimization. In: presented at the Aerospace Testing Expo 2005 North America, Long Beach Convention Center, Long Beach, CA, vol. AIAA-1999-1388. Nov 8-10 2005.

[2] GENESIS Users Manual: Design Manual, Version 9.0. Vanderplaats Research and Development, Inc., Colorado Springs, 2006.

[3] Vanderplaats, G.N.: Numerical Optimization Techniques for Engineering Design. 4th edn. Vanderplaats Research and Development, Inc., 2005.

[4] Maxwell, C.: Scientific Papers, vol. 2. Dover Publications, New York, 1952.

[5] Michell, A.G.M.: The Limits of Economy of Material in Frame Structures. Philosophical Magazine, vol. 8, no. 47, pp. 589-597, 1904.

[6] Shanley, F.R.: Weight Strength Analysis of Aircraft Structures. McGraw-Hill, New York, 1952.

[7] Heymann, J.: Plastic Design of Beams and Frames for Minimum Material Consumption. Quarterly of Applied Mathematics, vol. 8, pp. 373-381, 1951.

[8] Schmit, L.A.: Structural Design by Systematic Synthesis. In: 2nd Conference on Electronic Computation, American Society of Civil Engineers, New York, pp. 105-132. 1960.

[9] Schmit, L.A. and Farshi, B.: Some Approximation Concepts for Structural Synthesis. AAIA Journal, vol. 12(5), pp. 692-699, 1974.

[10] Schmit, L.A. and Miura, H.: Approximation Concepts for Efficient Structural Synthesis. NASA CR-2552, 1976.

[11] Vanderplaats, G.N. and Salajegheh, E.: A New Approximation Method for Stress Constraints in Structural Synthesis. AAIA Journal, vol. 27, no. 3, pp. 352-358, 1989.

[12] Canfield, R.A.: High Quality Approximations of Eigenvalues in Structural Optimization of Trusses. AAIA Journal, vol. 28, no. 6, pp. 1116-1122, 1990. 
[13] Leiva, J.P., Watson, B.C., Kosaka, I. and Vanderplaats, G.N.: Dynamic Finite Element Analysis and Optimization in GENESIS. In: 9th AIAA/ISSMO Symposium on Multidisciplinary Analysis and Optimization, Atlanta, Georgia. September 4-6, 2002.

[14] Leiva, J.P.: Topometry Optimization: A New Capability to Perform Element by Element Sizing Optimization of Structures. In: 10th AIAA/ISSMO Symposium on Multidisciplinary Analysis and Optimization, Albany, NY. August 30 - September $1,2004$.

[15] GENESIS Users Manual: Optimization Demonstration Problems, Version 9.0. Vanderplaats Research and Development, Inc., Colorado Springs, 2006.

[16] Candan, S., Garcelon, J., Balabanov, V. and Venter, G.: Shape Optimization using Abaqus and VisualDOC. In: 8th AIAA/USAF/NASA/ISSMO Symposium at Multidisciplinary Analysis and Optimization, Long Beach, CA. September 6-8, 2000.

[17] GENESIS/I-DEAS Interface User Manual: VMA Engineering, July, 1992.

[18] Kodiyalam, S., Vanderplaats, G.N. and Miura, H.: Structural Shape Optimization with MSC/NASTRAN. In: AIAA/ASME/ASCE/AHS/ASC 31St Structures, Structural Dynamics, and Materials Conference, Long Beach, CA, pp. 150-160. April 2-4, 1990.

[19] Leiva, J.P. and Watson, B.C.: Automatic Generation of Basis Vectors for Shape Optimization in the GENESIS Program. In: rth AIAA/USAF/NASA/ISSMO Symposium on Multidisciplinary Analysis and Optimization, St. Louis, MO, pp. 1115-1122. September 2-4, 1998.

[20] Leiva, J.P., Watson, B.C. and Kosaka, I.: Modern Structural Optimization Concepts Applied to Topology Optimization. In: 40th AIAA/ASME/ASCE/AHS/ASC Structures, Structural Dynamics, and Materials Conference, St. Louis, MO, vol. AIAA-1999-1388, pp. 1589-1596. Apr 12-15, 1999.

[21] Leiva, J.P.: Industrial Applications Using Structural Optimization with GENESIS. In: 4th World Congress of Structural and Multidisciplinary Optimization, Dalian, China. June 4-8, 2001.

[22] Leiva, J.P., Wang, L., Recek, S. and Watson, B.C.: Automobile Design Using the GENESIS Structural Optimization Program. In: Nafems Seminar: Advances in Optimization Tecnologies for Product Design, Chicago, Ilinois, USA. October 22-23, 2001. 
[23] Bendsoe, M.P. and Kikuchi, N.: Generating Optimal Topologies in Structural Design Using a Homogenization Method. Comp. Meth. Appl. Mech. Eng., vol. 71, pp. 197-224, 1988.

[24] Jog, C.S., Haber, R.B. and Bendsoe, M.P.: Topology Design with Optimized, Selfadaptive Materials. Int. Jour. for Mum. Meth. in Eng., vol. 37, pp. 1323-1350, 1994.

[25] Bendsoe, M.P.: Optimal Shape Design as a Material Distribution Problem. Structural Optimization, vol. 1, pp. 193-202, 1989.

[26] Swan, C.C. and Kosaka, I.: Reuss and Voigt Mixing Rules for Variable Topology Material Layout: Linear Elasticity. Int. Jour. for Mum. Meth. in Eng., vol. 40, pp. 3033-3057, 1997.

[27] Wang, B.P., Lu, C.M. and Yang, R.J.: Topology Optimization using MSC/NASTRAN. Technical paper, Ford Motor Company.

[28] Leiva, J.P.: Methods for Generating Perturbation Vectors for Topography Optimization of Structures. In: 5th World Congress of Structural and Multidisciplinary Optimization, Venice, Italy. May, 19-23, 2003.

[29] Bauermeister, H.: KAT Antenna Structure Design and Analysis. Tech. Rep. MMS document number: 2076-2814-23, IST document number: KAT7200PR10070037002, MMS Technology (Pty) Ltd, 1017 Kruger Avenue, Lyttelton Manor, Centurion, South Africa, August 31, 2006.

[30] Black, J.T. and Kohser, A.: De Garma's Material Processes and Manufacturing. Tenth edn. John Wiley \& Sons, 2008.

[31] MacDonald, A.J.: Wind Loadings on Buildings. Applied Science Publishers LTD, Ripple Road, Barking, Essex, England, 1975.

[32] Munson, B.R., Young, D.F. and Okiishi, T.H.: Fundamentals of Fluid Mechanics. 5th edn. John Wiley \& Sons, 2006.

[33] Venter, G. and Watson, B.: Efficient Optimization Algorithms for Parallel Applications. In: AIAA/USAF/NASA/ISSMO Symposium on Multidisciplinary Analysis and Optimization, 8th, Long Beach, CA, vol. AIAA-2000-4819. September 6-8, 2000 .

[34] Genberg, V.: Optical surface evaluation. In: Proceedings of the SPIE Symposuim on Structural Mechanics of Optical Systems. Nov 1983.

[35] Haftka, R.T. and Gürdal, Z.: Elements of Structural Optimization. 3rd edn. Kluwer Academic Publishers, P.O. Box 17, 3300 AA Dordrecht, Netherlands, 1993. 
[36] Taylor, J.E. and Bendsøe, M.P.: An Interpretation for Min-Max Structural Design Problems Including a Method for Relaxing Constraints. International Journal of Solids and Structures, pp. 301-314, 1984.

[37] Leiva, J.P. and Watson, B.C.: Buckling Finite Element Analysis and Optimisation in GENESIS. In: 8th Symposium on Multidisciplinary Analysis and Optimization, Long Beach, CA. September, 6-8, 2000. 